\title{
The Survey of Water and Ammonia in the Galactic Center (SWAG): Molecular Cloud Evolution in the Central Molecular Zone
}

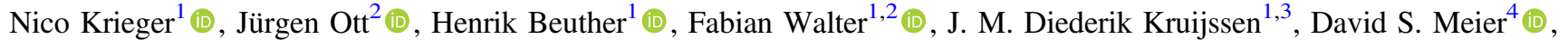

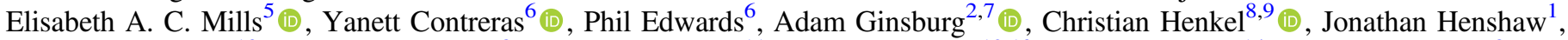 \\ James Jackson ${ }^{10}$ (1) , Jens Kauffmann ${ }^{8}$, Steven Longmore ${ }^{11}$ (D), Sergio Martín ${ }^{12,13}$, Mark R. Morris ${ }^{14}$ (), Thushara Pillai ${ }^{8}$, \\ Matthew Rickert ${ }^{15}$, Erik Rosolowsky ${ }^{16}$ (i), Hiroko Shinnaga ${ }^{17}$ (iD), Andrew Walsh ${ }^{18}$, Farhad Yusef-Zadeh ${ }^{15}$, and Qizhou Zhang ${ }^{19}$ (B) \\ ${ }^{1}$ Max-Planck-Institut für Astronomie, Königstuhl 17, D-69120 Heidelberg, Germany; krieger@mpia.de \\ ${ }^{2}$ National Radio Astronomy Observatory, P.O. Box O, 1003 Lopezville Road, Socorro, NM 87801, USA \\ ${ }^{3}$ Astronomisches Rechen-Institut, Zentrum für Astronomie der Universität Heidelberg, Mönchhofstraße 12-14, D-69120 Heidelberg, Germany \\ ${ }^{4}$ New Mexico Institute of Mining and Technology, 801 Leroy Place, Socorro, NM 87801, USA \\ ${ }^{5}$ San Jose State University, 1 Washington Square, San Jose, CA 95192, USA \\ ${ }^{6}$ Leiden Observatory, Leiden University, P.O. Box 9513, NL-2300 RA Leiden, The Netherlands \\ ${ }^{7}$ European Southern Observatory, Karl-Schwarzschild-Straße 2, D-85748 Garching bei München, Germany \\ ${ }^{8}$ Max-Planck-Institut für Radioastronomie, Auf dem Hügel 69, D-53121 Bonn, Germany \\ ${ }^{9}$ Astronomy Department, King Abdulaziz University, P.O. Box 80203, Jeddah 21589, Saudi Arabia \\ ${ }^{10}$ School of Mathematical and Physical Sciences, University of Newcastle, University Drive, Callaghan NSW 2308, Australia \\ ${ }^{11}$ Astrophysics Research Institute, Liverpool John Moores University, 146 Brownlow Hill, Liverpool L3 5RF, UK \\ ${ }^{12}$ European Southern Observatory, Alonso de Córdova 3107, Vitacura Casilla 763 0355, Santiago, Chile \\ ${ }_{13}$ Joint ALMA Observatory, Alonso de Córdova 3107, Vitacura Casilla 763 0355, Santiago, Chile \\ ${ }_{15}^{14}$ Department of Physics and Astronomy, UCLA, 430 Portola Plaza, Los Angeles, CA 90095-1547, USA \\ 15 Department of Physics and Astronomy and CIERA, Northwestern University, Evanston, IL 60208, USA \\ ${ }^{16}$ Department of Physics, CCIS 4-181, University of Alberta, Edmonton, Alberta, T6G 2E1, Canada \\ ${ }^{17}$ Department of Physics and Astronomy, Kagoshima University, 1-21-35, Korimoto, Kagoshima, 890-0065, Japan \\ ${ }_{18}^{18}$ International Centre for Radio Astronomy Research, Curtin University, Bentley, 6102, Australia \\ ${ }^{19}$ Harvard-Smithsonian Center for Astrophysics, 60 Garden Street, Cambridge, MA 02138, USA \\ Received 2017 July 5; revised 2017 October 17; accepted 2017 October 18; published 2017 November 20
}

\begin{abstract}
The Survey of Water and Ammonia in the Galactic Center (SWAG) covers the Central Molecular Zone (CMZ) of the Milky Way at frequencies between 21.2 and $25.4 \mathrm{GHz}$ obtained at the Australia Telescope Compact Array at $\sim 0.9 \mathrm{pc}$ spatial and $\sim 2.0 \mathrm{~km} \mathrm{~s}^{-1}$ spectral resolution. In this paper, we present data on the inner $\sim 250 \mathrm{pc}(1.4)$ between Sgr C and Sgr B2. We focus on the hyperfine structure of the metastable ammonia inversion lines $(J, K)=(1,1)-(6,6)$ to derive column density, kinematics, opacity, and kinetic gas temperature. In the CMZ molecular clouds, we find typical line widths of $8-16 \mathrm{~km} \mathrm{~s}^{-1}$ and extended regions of optically thick $(\tau>1)$ emission. Two components in kinetic temperature are detected at $25-50 \mathrm{~K}$ and $60-100 \mathrm{~K}$, both being significantly hotter than the dust temperatures throughout the CMZ. We discuss the physical state of the CMZ gas as traced by ammonia in the context of the orbital model by Kruijssen et al. that interprets the observed distribution as a stream of molecular clouds following an open eccentric orbit. This allows us to statistically investigate the time dependencies of gas temperature, column density, and line width. We find heating rates between $\sim 50$ and $\sim 100 \mathrm{~K} \mathrm{Myr}^{-1}$ along the stream orbit. No strong signs of time dependence are found for column density or line width. These quantities are likely dominated by cloud-to-cloud variations. Our results qualitatively match the predictions of the current model of tidal triggering of cloud collapse, orbital kinematics, and the observation of an evolutionary sequence of increasing star formation activity with orbital phase.
\end{abstract}

Key words: Galaxy: center - evolution - ISM: clouds - ISM: kinematics and dynamics - stars: formation

\section{Introduction}

The Galactic Center (GC) and the Central Molecular Zone (CMZ) in particular represent an environment with conditions that are not to be found anywhere else on large scales in the Milky Way. The CMZ received its name from the presence of a large reservoir of dense $\left(\gtrsim 10^{4} \mathrm{~cm}^{-2}\right)$ and molecular gas of a few times $10^{7} M_{\odot}$ (Oka et al. 1998; Morris \& Serabyn 1996; Ferrière et al. 2007) and covers the central $\sim 500$ pc of the GC region. The large amount of molecular gas is found to be accompanied by a relatively high star formation rate (SFR) of $\sim 0.1 M_{\odot} \mathrm{yr}^{-1}$ (Longmore et al. 2013a; Barnes et al. 2017). Star formation (SF) laws that build on the assumption of a constant gas-depletion time of $1 \mathrm{Gyr}$ can fit the observed SFR (Bigiel et al. 2010; Leroy et al. 2008, 2015). However, these relations are derived from gas at much lower densities than observed in the CMZ. Density-dependent SF laws, on the other hand, strongly overpredict the SFR (Longmore et al. 2013a) at $\sim 0.4 M_{\odot} \mathrm{yr}^{-1}$ (Kennicutt 1998; Krumholz \& McKee 2005; Krumholz et al. 2012) to $\sim 0.8 M_{\odot} \mathrm{yr}^{-1}$ Lada et al. (2010, 2012). Thus, the star formation efficiency (SFE) in the $\mathrm{CMZ}$ is significantly lower than expected for the observed gas densities. The complex interplay of energetic processes in the GC allows for different potential answers to this problem of low SFE, but none of these processes alone can explain the discrepancy (Kruijssen et al. 2014). A general picture of episodic starbursts in gas rings in galactic centers introduced by Krumholz \& Kruijssen (2015) and Krumholz et al. (2017) might solve the SFE problem in the particular case of the CMZ and set the framework for another intriguing feature of the GC: a ring-like structure of dust and molecular gas. This structure 


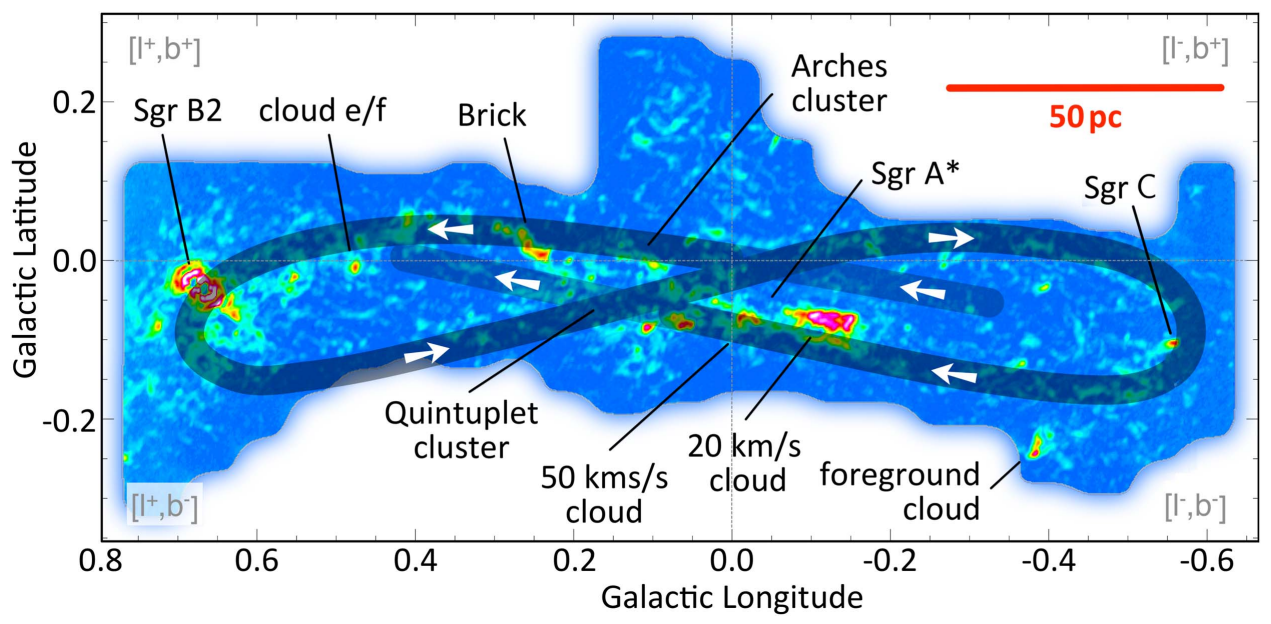

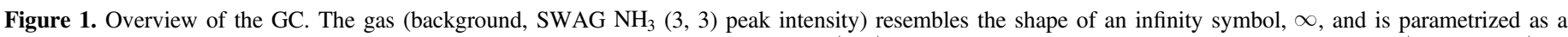

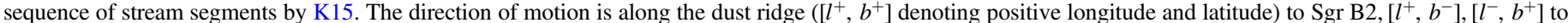

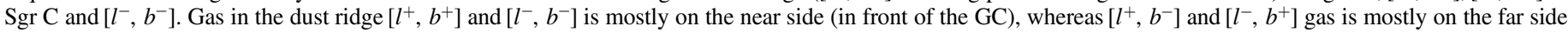
(Bally et al. 2010; Molinari et al. 2011). The most important sources are labeled.

(see Figure 1 for an overview) is projected onto an infinity $(\infty)$ shape that follows several arcs, the most prominent being the so-called "dust ridge" stretching from the massive molecular cloud G0.253+0.016 ("the Brick") to the star-forming region Sgr B2 (Lis et al. 1999). It might be continued via an $\left[l^{+}, b^{-}\right]$ arc and another loop at negative longitude $\left(\left[l^{-}, b^{+}\right],\left[l^{-}, b^{-}\right]\right)$ passing through the star-forming region Sgr C. The syntax $\left[l^{+/-}, b^{+/-}\right]$denotes the four quadrants in Galactic coordinates at positive/negative Galactic longitude (l) and latitude (b). Clouds at $\left[l^{-}, b^{-}\right]$and $\left[l^{+}, b^{+}\right]$are thought to be located in front of $\operatorname{Sgr~} \mathrm{A}^{*}$, i.e., the near side of the GC that is visible in silhouette against the background, whereas most of the $\left[l^{-}, b^{+}\right]$ and $\left[l^{+}, b^{-}\right]$gas is more likely to be on the far side, i.e., behind Sgr A* (Bally et al. 2010; Molinari et al. 2011).

Various models of the gas and dust distribution in the GC exist: a simple bar model (Morris \& Serabyn 1996); variations of a spiral arm model in which the apparent ring is formed by the inner part of two spiral arms (Sofue 1995; Sawada et al. 2004; Rodríguez-Fernández \& Combes 2008; RodríguezFernández 2011; Ridley et al. 2017); a closed, twisted elliptical ring detected in dust emission (Molinari et al. 2011), and a sequence of open-ended gas streams (Kruijssen et al. 2015, hereafter K15). K15 highlight the impossibility of closed orbits in extended gravitational potentials and provide a better fit to single-dish ammonia emission in position-position-velocity (PPV) space, as was confirmed by Henshaw et al. (2016b) for three molecular species in single-dish observations. Thus, we focus on this model and call it the "stream model" in contrast to the "ring model" and the "spiral arm" model. According to the stream model, the stream of GC molecular clouds oscillates radially and vertically, and can be traced for $\sim 1.5$ revolutions around the GC. The radial oscillation periodically brings dense molecular gas closer ( $r \sim 60 \mathrm{pc}$ at pericenter) to the gravitational center (traced by $\operatorname{Sgr} \mathrm{A}^{*}$ ) and deeper into the potential, whereas the apocenter lies at $r \sim 120 \mathrm{pc}$. As the CMZ clouds slowly evolve toward low virial ratios as the turbulent energy dissipates (Krumholz \& Kruijssen 2015; Walker et al. 2015; Henshaw et al. 2016a), it is statistically more likely that cloud collapse occurs at the pericenter where the compressive tidal forces are strongest along the orbit and thus a cloud receives the final nudge for the transition to self-gravitation. Subsequent
SF stages will then occur downstream from the pericenter passages and could potentially be observed as an SF sequence if the orbit is sufficiently sampled with molecular clouds that start to collapse at a similar point of their orbits. This model of triggered star formation was first proposed by Longmore et al. (2013b) based on the observation of different evolutionary SF stages along the dust ridge. The two young stellar clusters in the GC, Arches and Quintuplet, may also support this model as their orbits and ages are consistent with formation at a common point after collapse of their respective parent clouds at a pericenter passage (Figure 1 of Stolte et al. 2014; Kruijssen et al. 2015). In addition to stars and star formation tracers, cloud properties are also expected to show evolutionary behavior along the gas streams; this has not been thoroughly tested yet. Weak hints toward rising gas temperatures in the dust ridge are suggested by Ginsburg et al. (2016), while a recent paper by Kauffmann et al. (2017b) based on $\mathrm{N}_{2} \mathrm{H}^{+}$data from the Galactic Center Molecular Cloud Survey can neither confirm nor exclude the possibility of triggered evolution when examining mass-size relation and SF suppression. Both analyses were based on a low number of measurements $(\sim 35$ measurements in dust ridge clouds in Ginsburg et al. 2016 and six clouds in Kauffmann et al. 2017b) and lack the statistical power to detect potential evolution in the presence of scatter.

In this paper, we aim to test the prediction of the stream model by searching for coherent temporal evolution of molecular gas properties on a broad statistical basis using the Survey of Water and Ammonia in the Galactic Center (SWAG). SWAG targets 42 molecular and atomic species at $\sim 21-25 \mathrm{GHz}$, of which at least 20 are detected, and thus it offers an ideal database for such an analysis. SWAG maps the CMZ in the region of $\sim-1^{\circ}$ to $\sim+2^{\circ}$ in Galactic longitude at latitudes $|b|<0.4$ using the Australia Telescope Compact Array (ATCA) and achieves a spectral resolution of $0.4 \mathrm{~km} \mathrm{~s}^{-1}$ at $\sim 20^{\prime \prime}$ spatial resolution. One of the survey's signature targets is the ammonia molecule, the properties of which are well suited to exploring the thermal and kinematic structure of the interstellar medium (ISM; Ho \& Townes 1983). In an ammonia $\left(\mathrm{NH}_{3}\right)$ molecule, the nitrogen atom can tunnel through the plane of hydrogen atoms (inversion) at a rotational-state-dependent frequency that can be related to temperature. These inversion frequencies are closely spaced 
$\left(\Delta \nu_{(1,1) \ldots(6,6)} \sim 1.4 \mathrm{GHz}\right)$ while covering a large energy range (excitation energy $22<T_{l}[\mathrm{~K}] \lesssim 400$ ) that traces moderately dense gas of $10^{4}-10^{6} \mathrm{~cm}^{-3}$ due to the critical density being $n_{\text {crit }} \sim 10^{3} \mathrm{~cm}^{-3}$ (e.g., Shirley 2015). Detections of ammonia span a large range of sources from circumstellar envelopes (e.g., Betz et al. 1979) to galactic dark clouds (e.g., Ho et al. 1978) and nearby galaxies such as NGC 253 (e.g., Ott et al. 2005; Lebrón et al. 2011; Mangum et al. 2013; Gorski et al. 2017) to heavily star-forming galaxies like ultraluminous infrared galaxies (ULIRGS; e.g., Arp 220, Ott et al. 2011; Zschaechner et al. 2016) and even at high redshift $(z=0.9)$ as absorption against continuum emission (Henkel et al. 2008). In the GC, SWAG offers high-resolution $(\sim 0.9 \mathrm{pc})$ interferometric ammonia maps that show the intermediate-density gas structure most relevant to the stream model because large-scale diffuse emission $\gtrsim 1 ! 4(\gtrsim 3.3 \mathrm{pc} \text { at } 8.3 \mathrm{kpc})^{20}$ is filtered out by the ATCA interferometer in this setup. Spectral fitting of the ammonia hyperfine structure allows us to construct maps of lineof-sight velocity, line width, opacity, and kinetic gas temperature that can be tested for evolutionary behavior under the assumption of a kinematic model. Applying this method to SWAG data and the stream model allows us to check gas properties for absolute time dependencies that can be expected if a sequential star formation sequence is present in one or more of the stream segments.

As the structures in the GC are complex, simple names can be misleading. Throughout this paper, we refer to the "ring" as the general $\infty$-like structure of CMZ gas without being interested in the detailed structure or kinematics. "Stream," however, refers to the K15 model that introduced a stream as a set of four segments of an orbit wrapping around the GC whose subdivision does not have physical meaning but simplifies descriptions.

In this paper, we summarize the observational setup and data reduction of the SWAG survey in Section 2 on the basis of observations of one-third of the total area, covering the region between Sgr B2 and Sgr C. Section 3 shows and discusses the primary data products (channel and moment maps, spectra, position-velocity diagrams), and Section 4 describes the fitting of the ammonia hyperfine structure inversion lines. In Section 5 we use the derived thermal and kinetic properties of GC molecular clouds to discuss their properties in the context of the orbital model of $\mathrm{K} 15$ by reconstructing potential time dependencies of these properties. The resulting relations are discussed in the context of the star formation sequence as proposed by Longmore et al. (2013b). A summary in Section 6 concludes this work. Appendices show further SWAG data products (Appendix A), details on imaging (Appendix B), the ammonia thermometer (Appendix $\mathrm{C}$ ), and hyperfine structure line fitting (Appendix D), as well as further fit-derived data products (Appendices $\mathrm{E}$ and $\mathrm{F}$ ) and further time evolution plots (Appendix G).

\section{Observations and Data Reduction}

SWAG is a large survey aiming to map the entire CMZ of the Milky Way from $\sim-1^{\circ}$ to $\sim+2^{\circ}$ in Galactic longitude at latitudes $|b|<0$. 4 . The boundaries correspond to an integrated surface brightness level of $0.1 \mathrm{~K} \mathrm{~km} \mathrm{~s}^{-1}$ of $\mathrm{NH}_{3}(3,3)$ from

\footnotetext{
${ }^{20}$ We adopt this distance measurement by Reid et al. (2014) throughout the paper. The IAU recommends to use $8.5 \mathrm{kpc}$, while a recent study by Boehle et al. (2016) found $7.86 \mathrm{kpc}$.
}

Mopra single-dish observations (beam size $\sim 2 ! 4$ Ott et al. 2014). The achieved spatial resolution is $\sim 22$ !" $8(20$ " $6-25$ !" 0 in the range of $25.4-21.2 \mathrm{GHz}$ ), which at the Galactic Center distance corresponds to $\sim 0.9 \mathrm{pc}$. The velocity resolution is $\sim 0.4 \mathrm{~km} \mathrm{~s}^{-1}\left(0.37-0.45 \mathrm{~km} \mathrm{~s}^{-1}\right.$ for $\left.25.4-21.2 \mathrm{GHz}\right)$. The observations were performed in a mosaic of $\sim 6500$ pointings with the ATCA interferometer during $\sim 525 \mathrm{hr}$ spread over three years (2014-2016). At a wavelength of around $1.3 \mathrm{~cm}, 42$ spectral lines were targeted in the range of $21.2-25.4 \mathrm{GHz}$; among these are six metastable and three non-metastable ammonia inversion lines, water maser and atomic radio recombination lines, and complex organic molecules. At least 20 of the targeted lines are unambiguously detected.

\subsection{Observations}

The observations for SWAG were carried out with the $\mathrm{ATCA}^{21}$ interferometer. Achieving the maximum sensitivity to extended emission requires the most compact array configuration, H75, with baselines of 31-89 $\mathrm{m}$ between five dishes. A sixth antenna that is not considered in this work provides baselines of $\sim 4.4 \mathrm{~km}$ to the inner antennas. The primary beam FWHM of the $22 \mathrm{~m}$ dishes at $\nu=23 \mathrm{GHz}$ is $\sim 2$ ! 4. Every position in the targeted area is covered by the primary beam of at least three pointings. In the radio $K$-band regime, the $\mathrm{H} 75$ array configuration is not sensitive to emission extending over more than $\sim 1$ ! 4 $(3.3 \mathrm{pc})$ because of interferometric spatial filtering.

Between 40 and 220 pointings are combined into strips of $\sim 4$.5 width, as shown in Figure 2, which are observed between subsequent observations of the phase calibrator. Long strips are split into Galactic northern and southern parts to ensure frequent phase calibrations. In order to maximize the $u, v$ coverage, pointings are not observed one after the other, but in rows of even and odd numbers, as explained in Ott et al. (2014). The rows are aligned parallel to Galactic longitude ("l-scan" in Ott et al. 2014), while the first element of each row determines its parity (odd, even). Rows of odd parity are observed consecutively, followed by the left-out rows of even parity at somewhat later local sidereal times (LST) and therefore other projected baselines, which increases the $u, v$ coverage. The resulting typical $u, v$ coverage of a single pointing is shown in Figure 3.

The Compact Array Broadband Backend (CABB, Wilson et al. 2011) provides two IF bands, each of $2 \mathrm{GHz}$ bandwidth. In the CFB 64M-32k mode adopted for these observations, each $2 \mathrm{GHz}$ band was divided into $32 \times 64 \mathrm{MHz}$ "continuum" channels with 16 "zoombands" of $2048 \times 32 \mathrm{kHz}$ channels selected in each IF band for spectral line observations. The integration time of each pointing was set to $8 \times 30 \mathrm{~s}=$ 4 minutes as a trade-off between low noise and total project time, but it varied due to reobservations and flagging. The eight individual integrations were scheduled for best $u, v$ coverage to be observed over the whole available range of LST from about 15:00 to $22: 00 \mathrm{hr}$ as much as possible. Owing to pointing overlap, the actual sensitivity is higher by a factor of $\sqrt{3}(\sqrt{2}$ for edge pointings) than can be expected from the integration time of a single pointing. The expected noise calculated from the radiometer equation $\sigma \propto \frac{T_{\text {sys }}}{\sqrt{\Delta \nu \tau}}$ as a function of system temperature $T_{\text {sys }}$, bandwidth $\Delta \nu$, and integration time $\tau$ is

\footnotetext{
21 The ATCA is part of the Australia Telescope National Facility, which is funded by the Australian Government for operation as a National Facility managed by CSIRO.
} 


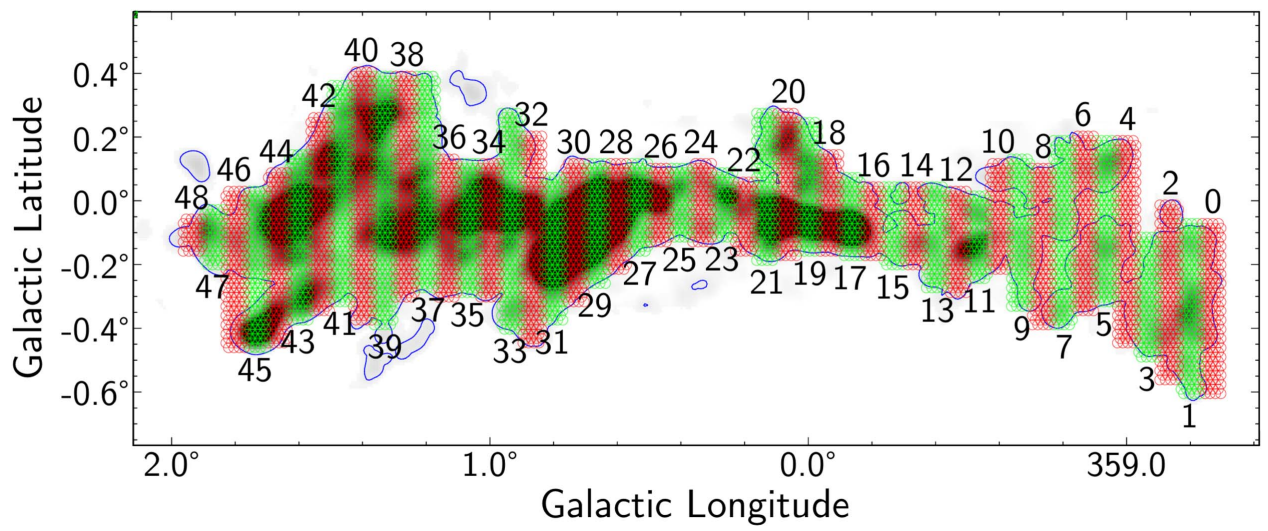

Figure 2. SWAG observing layout. The spatial coverage of SWAG is derived from the $0.1 \mathrm{~K} \mathrm{~km} \mathrm{~s}^{-1}$ contour (blue) of the Mopra single-dish CMZ survey by Ott et al. (2014; background gray-scale image). Between 40 to 220 pointings are included in an observation strip (numbered from west to east and alternately colored). This work includes data of the inner CMZ covered by strips 10 to 30 .

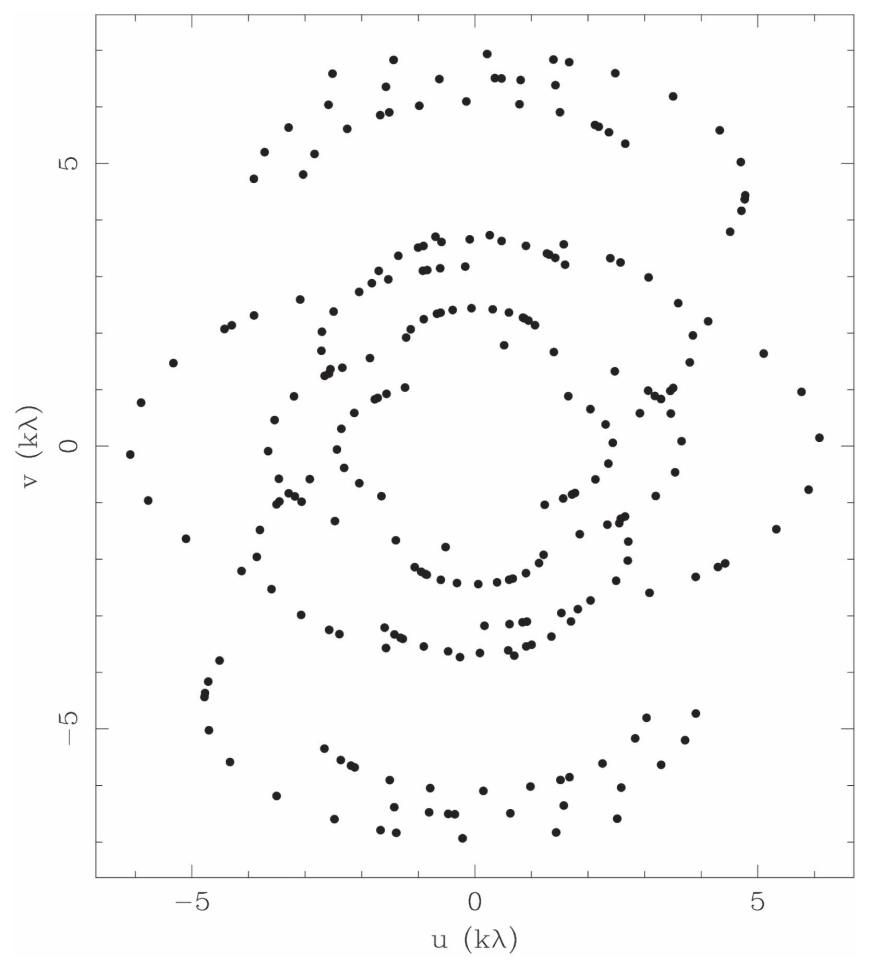

Figure 3. Typical $u, v$ coverage of a single pointing of SWAG using the inner array of five antennas (excluding the far out antenna ca06). Note that overlapping pointings increase the $u, v$ coverage by a factor of $\sqrt{3}(\sqrt{2}$ for edge pointings), which is not included in this plot. The $u, v$ coverage was optimized by the scanning technique ("l-scan") shown in Figure 1 of Ott et al. (2014).

$\sigma=28 \mathrm{mK}$ for a typical $T_{\text {sys }}=80 \mathrm{~K}$ in one channel of $\Delta \nu=32 \mathrm{kHz}\left(\sim 0.4 \mathrm{~km} \mathrm{~s}^{-1}\right.$ at $\left.23 \mathrm{GHz}\right)$ and $t=240 \mathrm{~s}$. This value corresponds to $\sigma \sim 6.0$ mJy beam $^{-1}$ for $\mathrm{NH}_{3}(3,3)$ $(\nu=23.87 \mathrm{GHz}$, beam 26 !" $03 \times 17$ !" 71$)$, which is about half of the measured noise of $\sim 13.1 \mathrm{mJy}^{-1}$ beam $^{-1}$ (Section 2.3.1) because the deconvolution is imprefect owing to the sparsely sampled $u, v$ plane.

Observations were scheduled in three-week blocks in the southern hemisphere winter months of July/August in each year from 2014 to 2016 . The total project time including calibration data sums up to $\sim 720 \mathrm{hr}(\sim 204 \mathrm{hr}$ for the data presented in this paper), including additional time needed to reobserve some regions whose observations were affected by bad weather, resulting in increased noise. The total on-source time was $\sim 525 \mathrm{hr}(\sim 150 \mathrm{hr}$ shown in this paper $)$.

Bandpasses and delay calibrations for each antenna are derived from daily 10-minute integrations on PKS 1253-055 (3C 279). Its flux of $\sim 16-20 \mathrm{Jy}$ at frequencies around $23 \mathrm{GHz}$ is bright enough to derive accurate bandpass solutions. The gain/phase (complex gain) calibrator PKS 1710-269 was observed for 2 minutes every 20-30 minutes, depending on the field, after each l-scan and in between l-scans if necessary. Daily flux calibrations of 5-minute integration time are performed on the radio galaxy PKS 1934638, which is the standard primary flux density calibrator for the ATCA below $50 \mathrm{GHz}$.

\subsection{Calibration}

All steps from data import to imaging are performed with the ATCA specific version of the package MIRIAD ${ }^{22}$ (Sault et al. 1995). The necessary steps were developed into a data reduction pipeline that provides the base for reducing the entire survey.

\subsubsection{Flagging}

Raw data are imported via atlod with options birdie, nocacal, noif, and opcorr to automatically flag resonant instrument modes (birdies), initial array calibration data (cacal), set correct intermediate-frequency mapping behavior, and to apply atmospheric opacity corrections. Some of the array setup data were not detected by the automatic routine and had to be flagged manually with uvflag.

Resonant modes in ATCA's backend system cause additional birdies in channels $n \times 1024+1(n=1,2, \ldots)$ that are not identified during data import. The missing channels are interpolated later when averaging and Doppler-correcting the visibilities to a common resolution of $2 \mathrm{~km} \mathrm{~s}^{-1}$. To remove additional correlator artifacts and extreme RFI, calibrator data were clipped at $200 \mathrm{Jy}$ and $\mathrm{CMZ}$ observations at $10 \mathrm{Jy}$. Additional flagging of bad data was made by eye in the interactive task blflag. Polarization measurements were not required for the presented analysis, and all crosshands/crosspolarizations (XY and YX for ATCA's linear feeds) were flagged. Antenna ca06 offers high spatial resolution at baselines of $>4300 \mathrm{~m}$, but was also flagged due to lack of

\footnotetext{
${ }^{22}$ http://www.atnf.csiro.au/computing/software/miriad
} 


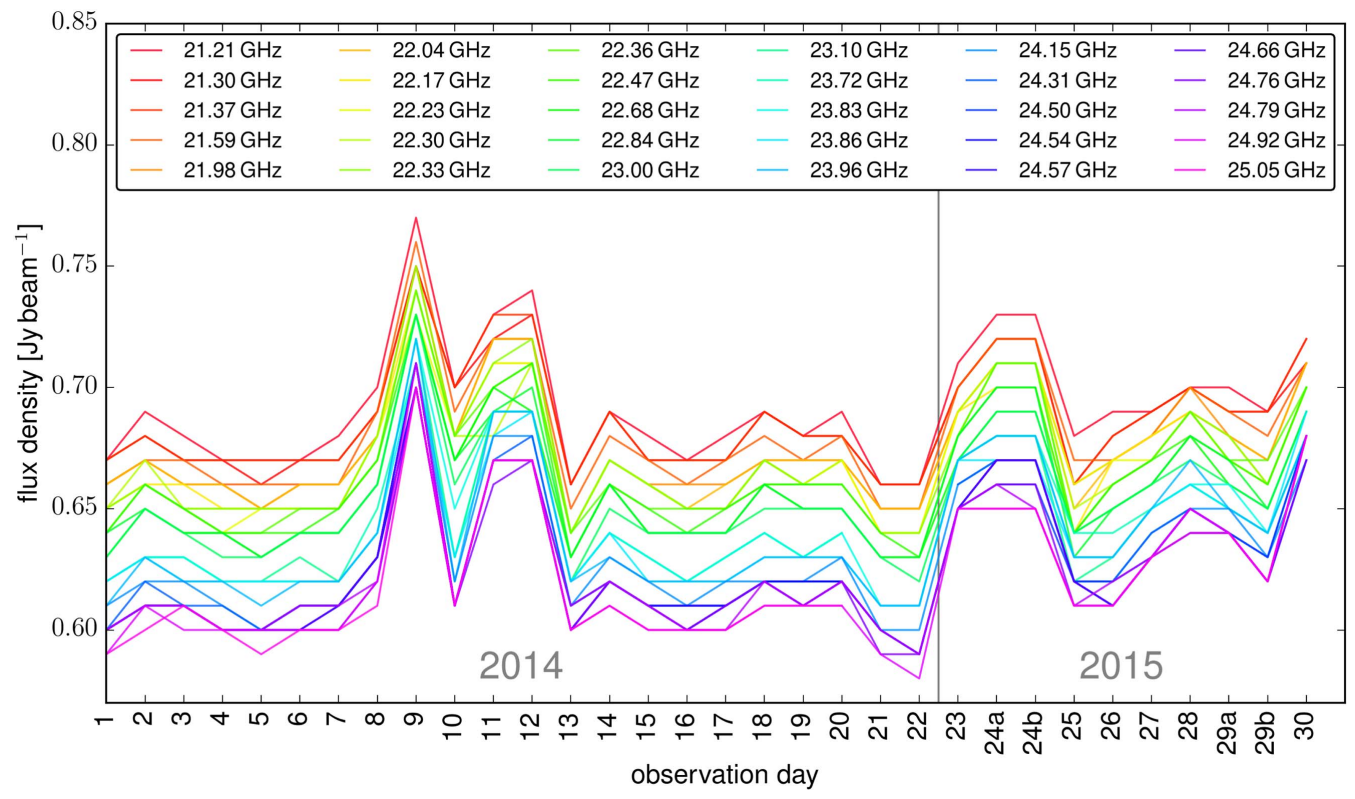

Figure 4. Flux density of the phase calibrator PKS 1710-269 per observation day after all calibration steps for the 30 frequency bands of CABB. On observation days 24 and 29, correlator crashes required new calibrations, which are labeled a and b and correspond to flux densities before and after the crash, respectively. Flux densities are constant across observation days within $\sim 15 \%$. The increasing values toward lower frequency is an intrinsic property of the source: a quasar with spectral index $\sim-0.5$. The precision of the MIRIAD task imstat is two decimal places, which leads to quantized data.

intermediate $u, v$ points, which drastically down-weights these long baselines. Except for the instrumental birdies we described, little radio frequency interference (RFI) is present at frequencies of $\sim 23 \mathrm{GHz}$.

\subsubsection{Complex Gain Calibration}

Bandpass amplitudes and phases are calculated on the designated bandpass calibrator PKS 1253-055. The resulting correction is then applied to the gain/phase calibrator PKS 1710-269 and flux density calibrator PKS 1934-638, and complex antenna gains are derived with mfcal.

Calibration solutions were inspected visually to identify remaining problems that were subsequently flagged. Recalibrations were performed until well-behaved solutions were found.

\subsubsection{Flux Density Calibration}

The calibration quality is assessed with Figure 4. The phase calibrator PKS 1710-269 was imaged including all calibration corrections for all observation days and all zoombands and the peak flux density listed. Measured flux densities per zoomband are constant within $\sim 15 \%$, which is typical for radio observations at this frequency. The origin of this variability cannot be identified without absolute measurements and can be due to changing atmospheric conditions or intrinsic luminosity variation in the QSO PKS 1710-269. Thus, $15 \%$ is an upper limit on the flux density variation across observation days, which is similar to the absolute flux uncertainty.

Spectral lines observed in the same zoomband, however, share a common flux density uncertainty, and thus flux ratios and derived quantities are more accurate. This provides a robust basis for the derivation of the kinetic gas temperature, which is primarily determined from ammonia line ratios.

\subsubsection{Continuum Subtraction}

Over the relatively small spectral range of a zoomband of $\sim 64 \mathrm{MHz}$, a linear approximation captures the spectral index of any continuum emission well enough. Line-free channels could not be identified in visibility spectra because the brightness of most of the spectral lines is too low; instead, they are calculated from the line's rest frequency and a window of \pm 400 channels (corresponding to $\sim 178-150 \mathrm{~km} \mathrm{~s}^{-1}$ at $21.0-25.0 \mathrm{GHz}$ ), which is large enough to exclude the typical velocity range of molecular gas in the CMZ of $\pm 150 \mathrm{~km} \mathrm{~s}^{-1}$. Any possible emission outside these line-free windows is weak and does not affect the continuum fit. Fit and subtraction were made using the uvlin task, forcing a first-order polynomial.

Rest frequencies are set according to Table 1, which lists values from splatalogue. ${ }^{23}$

Merging five channels to obtain a spectral resolution of $2 \mathrm{~km} \mathrm{~s}^{-1}$ increases the signal-to-noise ratio $(\mathrm{S} / \mathrm{N})$ by a factor of $\sqrt{5} \sim 2.2$, while the spectral resolution is still adequate for the expected typical line widths of $5-25 \mathrm{~km} \mathrm{~s}^{-1}$ in ammonia emission.

\subsection{Imaging}

\subsubsection{Deconvolution}

Fourier transformation from the visibility to the image domain is performed with the task invert, which includes primary beam correction and combines the visibilities of single pointings into a mosaic. The Briggs weighting parameter is set to +2 , which is close to natural weighting. The cell size (pixel size) is set to $5^{\prime \prime}$ in R.A. and decl., which oversamples the minor axis of the synthesized beam $\left(24\right.$ ! $^{\prime} 8 \times 16^{\prime \prime} .9,89^{\circ} .3$ at $25.1 \mathrm{GHz}$ ) by a factor $>3$. At lower frequency, the beam size grows up to 26 ". $2 \times 17$ ". 8 at $23.7 \mathrm{GHz}$, which results in a

\footnotetext{
${ }^{23}$ http://www.splatalogue.net
} 
Table 1

For Each Ammonia Transition (1), We Detail the (2) Rest Frequency, (3) Excitation Energy of the Upper Transition State, (4) Natural

Weighted Beam Sizes $\left(b_{\text {maj }} \times b_{\text {min }}\right)$, (5) Beam Position Angle, rms Noise for a Channel Width of $2 \mathrm{~km} \mathrm{~s}^{-1}$ in the Two Regions of (6) Normal $\left(\sigma_{\text {normal }}\right)$ and $(7)$ Elevated Noise $\left(\sigma_{\text {high }}\right)$

\begin{tabular}{lcccccc}
\hline \hline Transition & $\begin{array}{c}\text { Frequency } \\
(\mathrm{GHz})\end{array}$ & $\begin{array}{c}E_{u} / k_{\mathrm{B}} \\
(\mathrm{K})\end{array}$ & $\begin{array}{c}\text { Beam Size } \\
\left({ }^{\prime \prime}\right)\end{array}$ & $\begin{array}{c}\text { PA } \\
\left({ }^{\circ}\right)\end{array}$ & $\begin{array}{c}\sigma_{\text {normal }} \\
\left(\mathrm{mJy}^{2} \text { beam }^{-1}\right)\end{array}$ \\
\hline $\mathrm{NH}_{3}(1,1)$ & 23.69451 & 24.4 & $26.2 \times 17.8$ & 89.3 & 13.4 & 19.4 \\
$\mathrm{NH}_{3}(2,2)$ & 23.72263 & 65.6 & $26.2 \times 17.8$ & 89.3 & 13.1 & 19.3 \\
$\mathrm{NH}_{3}(3,3)$ & 23.87013 & 124.7 & $26.0 \times 17.7$ & 89.3 & 13.1 & 18.6 \\
$\mathrm{NH}_{3}(4,4)$ & 24.13942 & 201.7 & $25.7 \times 17.5$ & 89.3 & 12.9 & 16.1 \\
$\mathrm{NH}_{3}(5,5)$ & 24.53299 & 296.5 & $25.3 \times 17.2$ & 89.3 & 12.0 & 16.2 \\
$\mathrm{NH}_{3}(6,6)$ & 25.05603 & 409.2 & $24.8 \times 16.9$ & 89.3 & 13.2 & 16.2 \\
mean value & & & & & 13.0 & 17.6 \\
\hline
\end{tabular}

Note. $\sigma_{\text {normal }}$ is relevant for most of the maps beside the two outermost strips (see Figure 2) on each side that are described by $\sigma_{\text {high. Given values are an }}$ average over $20\left(\sigma_{\text {normal }}\right)$ and $12\left(\sigma_{\text {high }}\right)$ measurements in two channels at $\pm 150 \mathrm{~km} \mathrm{~s}^{-1}$ relative to the rest frequency.

variation of linear size of $\sim 5 \%$ between $\mathrm{NH}_{3}(1,1)$ and $(6,6)$. These beam size are $\sim 1.7$ and $\sim 1.2$ times the naively expected size of $\sim 15^{\prime \prime} \times 15^{\prime \prime}$ due to weighting. Table 1 lists the obtained beam sizes for $\mathrm{NH}_{3}(1,1)$ to $(6,6)$.

Each pointing is imaged over 512 pixel (FOV 42!7) and then integrated linearly into the mosaic with another weighting function that smooths noise across regions of differing pointing coverage (MIRIAD default method, Sault et al. 1996).

The extended emission of most spectral lines is generally better deconvolved by an algorithm that also uses extended sources for modeling. The only possibility of extended modeling that MIRIAD offers is the maximum entropy method (MEM). Our tests with the mosaic versions of MEM (mosmem) and clean (mossdi) on SWAG data confirmed that more extended emission was reconstructed by MEM over regular clean.

A first run with up to 10 iterations deconvolves the dirty image well enough to construct a mask that prevents treating regions without significant emission. Image restoration is done with restor. A mask is calculated to contain all pixels with $\mathrm{S} / \mathrm{N}$ above $\sim 5$, which corresponds to $65.0 \mathrm{mJy} \mathrm{beam}^{-1}$ in the semi-cleaned image.

Deconvolution is then repeated with 50 iterations in mosmem only inside the pixels that were set as relevant by the mask. The restored images still contain sidelobe structures, especially around strong sources and in strong lines. The difficulty of obtaining better images with mosmem is described in more detail in Appendix B.

The root mean square (rms) noise is not constant within and across the data cubes, but increases slightly with decreasing frequency because of the proximity to an atmospheric water line that increases the telescope's system temperature. At a level of $\sim 13.0 \mathrm{mJy}^{-1}$ beam $^{-1}$, the difference between the channels at $-150 \mathrm{~km} \mathrm{~s}^{-1}$ and $+150 \mathrm{~km} \mathrm{~s}^{-1}$ relative to rest frequency is $0.1-0.6 \mathrm{mJy}$ beam $^{-1}$ for the ammonia lines listed in Table 1. Thus, a common value is calculated as the mean of minimum and maximum noise measurements. The spatial variation of noise across pointings is negligible except for the two outermost strips of the data considered in this work $(10 / 11$ and 30/31, see Figure 2), which show increased noise levels in the 2014/2015 data that are due to bad weather. Hence, two values $\sigma_{\text {normal }}$ and $\sigma_{\text {high }}$ are needed to describe the noise properties in the inner map and the $l^{+} / l^{-}$edges, respectively. Table 1 lists these noise levels for the six metastable ammonia lines. Any further mention of noise refers to $\sigma_{\text {normal }}$ as only two major molecular clouds (Sgr B2 and Sgr C) are located in the region of elevated noise, and they are strong sources with high $\mathrm{S} / \mathrm{N}$.

\subsubsection{Masking of the Convolved Data Cubes}

The outer edges of all data cubes exhibit a $\sqrt{3}$ higher noise than the rest of the maps because of non-overlapping pointings. These edges are blanked from the edge inward by $\sim 50^{\prime \prime}$ and $\sim 100^{\prime \prime}$ ( one-third and two-thirds of the primary beam, respectively) as the smaller radius of $50^{\prime \prime}$ proved to be enough to exclude any visible higher noise edges in ammonia distribution maps, whereas spectral fitting is affected by increased noise to $\sim 90^{\prime \prime}$ from the edge.

\subsubsection{Moment Maps}

Data cubes were collapsed along the spectral axes with the task moment in MIRIAD to obtain moment maps. Map edges are blanked with the $50^{\prime \prime}$ edge mask and noise is excluded at levels of $3 \sigma$ and $5 \sigma$ for intensity maps (peak intensity; moment 0 , integrated intensity) and velocity maps (moment 1 , intensity-weighted mean velocity; moment 2 , velocity dispersion), respectively.

\subsubsection{Position-Velocity Diagrams}

Position-velocity cuts through the restored datacube edge masked at $100^{\prime \prime}$ were calculated with the task impv in the CASA software package ${ }^{24}$ (McMullin et al. 2007) along Galactic longitude, averaging over all emission in Galactic latitude.

\section{Results}

Figures in this section show $\mathrm{NH}_{3}(3,3)$ as representative of all six observed metastable ammonia inversion lines as it is typically the brightest ammonia line observed with SWAG. The corresponding figures for $\mathrm{NH}_{3}(1,1),(2,2),(4,4),(5,5)$, and $(6,6)$ can be found in Appendix A.

\subsection{Data Cubes}

Channel maps of $2 \mathrm{~km} \mathrm{~s}^{-1}$ width in steps of 15 channels $\left(30 \mathrm{~km} \mathrm{~s}^{-1}\right)$ are shown in Figure 5. All velocities are specified in the LSRK frame using the radio approximation. Emission can be detected in the range of $\sim-180 \mathrm{~km} \mathrm{~s}^{-1}$ to $\sim 150 \mathrm{~km} \mathrm{~s}^{-1}$ with emission related to the ring-like feature between $\sim-150 \mathrm{~km} \mathrm{~s}^{-1}$ and $\sim 120 \mathrm{~km} \mathrm{~s}^{-1}$. Extended gas clumps at $l \sim 0^{\circ}, b \sim 0.15$ are offset in velocity from the surrounding gas and are detected among the highest velocity gas in the field of view. Typical flux density values for molecular clouds in the $\mathrm{NH}_{3}(3,3)$ line are on the order of a few hundred $\mathrm{mJybeam}^{-1}$ with peaks of $4.23 \mathrm{Jy} \mathrm{beam}^{-1}(19.6 \mathrm{~K})$ in the $20 \mathrm{~km} \mathrm{~s}^{-1}$ cloud and $5.28 \mathrm{Jy} \mathrm{beam}^{-1}(24.8 \mathrm{~K})$ in Sgr B2. The spatial distribution is very similar among the ammonia lines, despite the varying flux density levels (Sections 5 and A.1).

\footnotetext{
${ }^{24}$ Common Astronomy Software Applications, https://casa.nrao.edu/.
} 


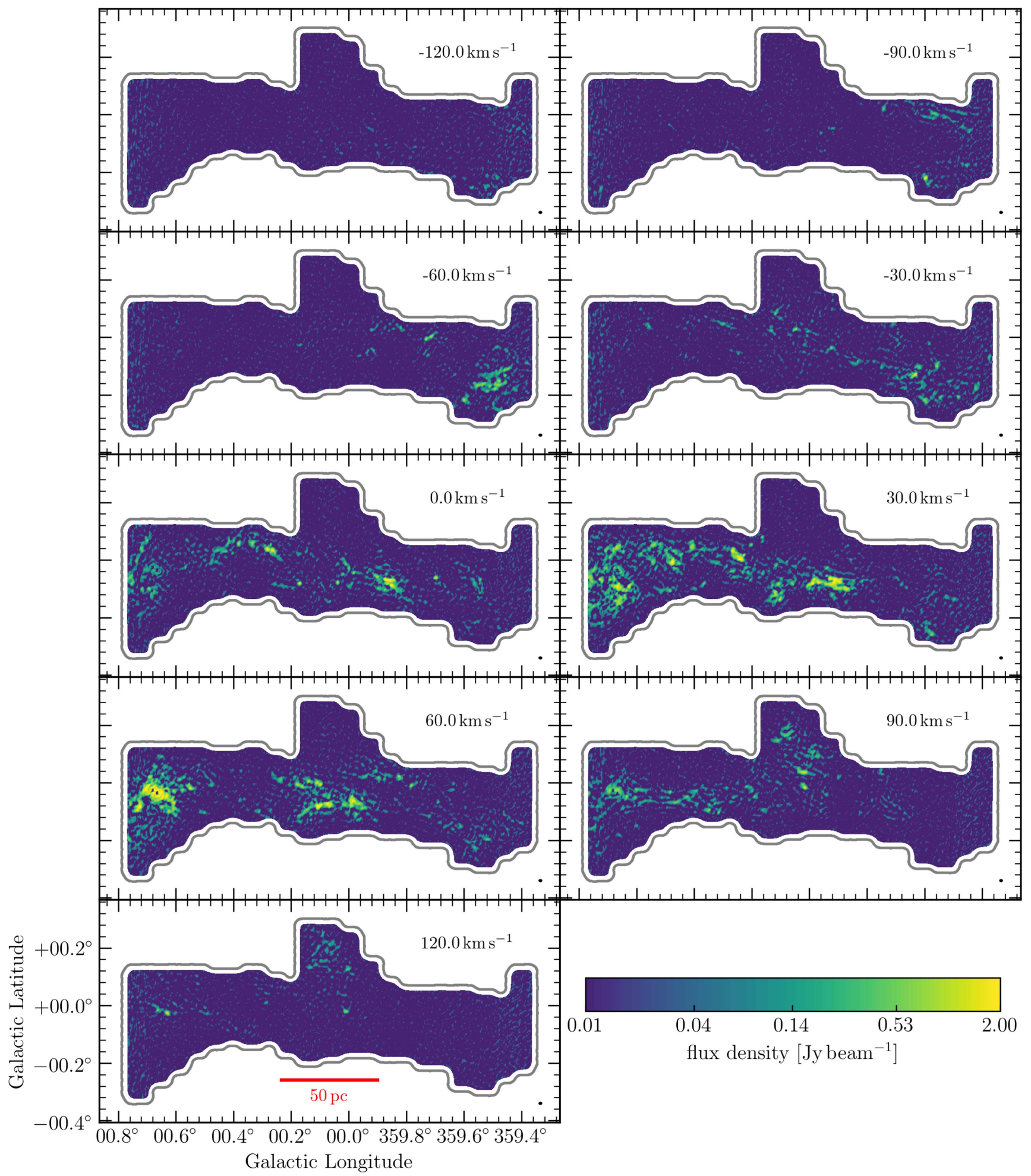

Figure 5. Channel maps of $\mathrm{NH}_{3}(3,3)$. Every fifteenth channel of $2 \mathrm{~km} \mathrm{~s}^{-1}$ (separated by $30 \mathrm{~km} \mathrm{~s}^{-1}$ ) in the range of $-120 \mathrm{~km} \mathrm{~s}^{-1}$ to $+120 \mathrm{~km} \mathrm{~s}^{-1}$ is shown. Velocity and beam $\left(26^{\prime \prime}, 03 \times 17^{\prime \prime} .71,89^{\circ} .3\right)$ are indicated in the top and bottom right corners, respectively. The conversion factor from flux density to brightness temperature for this beam size at $23.87 \mathrm{GHz}$ is $T[\mathrm{~K}] / S\left[\mathrm{Jy} \mathrm{beam}^{-1}\right]=4.65$.

\subsection{Sample Spectra}

Depending on the structure of the source and the spectral line, hyperfine satellite components and multiple components along the line of sight are detected. A bright single-component example spectrum of $\mathrm{NH}_{3}(3,3)$ is shown in Figure 6 toward the Brick
(G0.253+0.016). Absorption against continuum supposedly from free-free emission is detected toward the $50 \mathrm{~km} \mathrm{~s}^{-1}$ cloud, the Brick, clouds $\mathrm{c}$ and $\mathrm{f}$, and some smaller clouds in the $l^{+}, b^{-}$and $l^{-}, b^{+}$portions of the "ring" (cf. Figure 1).

Further spectra at the same position in the Brick for $\mathrm{NH}_{3}(1,1)$ to $(6,6)$ are shown in Appendix A.2. 


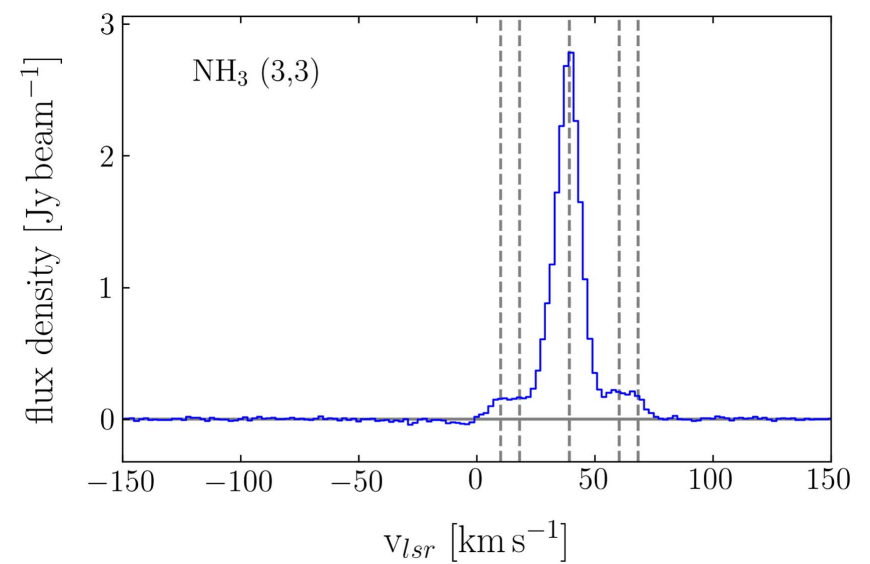

Figure 6. Sample spectrum toward the Brick $(l=0.2328, b=0.0107)$ extracted from the $\mathrm{NH}_{3}(3,3)$ cube. rms noise is $13.1 \mathrm{mJy}^{\text {beam }}{ }^{-1}(0.061 \mathrm{~K})$. The positions of the hyperfine satellites according to Table 3 are indicated by dashed vertical lines. Note the broad line width that blends the outer hyperfine components (which have separations of $\pm 21.1 \mathrm{~km} \mathrm{~s}^{-1}$ and $\pm 29.1 \mathrm{~km} \mathrm{~s}^{-1}$ from the main line) into a pedestal.

\subsection{Image Moments}

Image moment maps are shown in Figure 7 for orders 0 (integrated intensity), 1 (velocity centroid), and 2 (velocity dispersion); the top panel shows the peak intensity.

Peak intensity (Figure 7(a)) and integrated intensity (Figure 7(b)) show an almost identical distribution of emission, with Sgr $\mathrm{C}$ being a notable exception of strong peak but low integrated intensity, indicating narrow line widths. Sgr B2, the arching structure of the dust ridge $(0.2<l<0.6)$ and the sequence of massive clouds at $-0^{\circ} .2<l<0.15$ with a bifurcation $^{25}$ at $l \sim 0^{\circ} .1$ are most prominent in both intensity maps. The line-of-sight velocity (Figure 7(c)) shows a complex pattern at $0.0<l<0$. 2 of multiple partially overlapping emission components along the line of sight. At negative longitudes, the coherent structure of negative velocities is intermingled with clouds at positive velocities. In total, the molecular arcs trace an apparently ring-like structure. As the interferometer filters out diffuse emission, the molecular clouds in this ring-like structure must be rather clumpy, which has been reported previously (Bally et al. 2010; Molinari et al. 2011; Ott et al. 2014; Ginsburg et al. 2016).

Using the definition $l^{+/-}$and $b^{+/-}$denoting positive/negative values of Galactic longitude and latitude, the general sense of rotation in the CMZ is consistent with the scenario $\left[l^{-}, 0\right] \rightarrow$ $\left(\left[l^{-}, b^{-}\right] \rightarrow[0,0] \rightarrow\left[l^{+}, b^{+}\right] \rightarrow\left[l^{+}, 0\right] \rightarrow\left[l^{+}, b^{-}\right] \rightarrow[0,0] \rightarrow\right.$ $\left[l^{-}, b^{+}\right]$found in previous studies (e.g., Molinari et al. 2011; Kruijssen et al. 2015; Ginsburg et al. 2016). This, however, does not necessarily mean that arcs are physically connected, e.g., note the $50 \mathrm{~km} \mathrm{~s}^{-1}$ jump between $\left[l^{-}, b^{-}\right]$and $\left[l^{+}, b^{+}\right]$, but indicates that the bulk of the gas is following the same sense of rotation. Typical velocity dispersions in the moment- 2 map are found to be $5-25 \mathrm{~km} \mathrm{~s}^{-1}$, but velocity dispersions for individual clouds are expected to be lower because of multiple components along the line of sight, and for the lower excitation states, blending of hyperfine components.

Moment maps of $\mathrm{NH}_{3}(1,1)$ to $(6,6)$ are shown in Appendix A.3.

\footnotetext{
${ }^{25}$ This term is adopted from K15 and denotes the apparent splitting after the $50 \mathrm{~km} \mathrm{~s}^{-1}$ cloud when moving toward positive longitudes, best seen in Figure 5 at $60 \mathrm{~km} \mathrm{~s}^{-1}$.
}

\subsection{Structure in Position-Velocity Space}

A position-velocity diagram along Galactic longitude integrated over Galactic latitude traces the ring-like structure in the CMZ (Figure 8), which can be seen as roughly parallel structures traversing the diagram diagonally. A detailed analysis of the spatial and kinematic structure is given in Section 4.2 .2 based on fit-derived kinematics.

\section{Analysis: Pixel-by-pixel Hyperfine Structure Fitting}

Several basic cloud properties can be derived from the metastable ammonia inversion lines (Ho \& Townes 1983). The tunneling frequency of the nitrogen atom through the plane of three hydrogen atoms (inversion) depends on the rotational state $(J, K)$ of the molecule. The population of these states is temperature dependent according to a Boltzmann law. For each rotational state $J$ (angular momentum), there exists another ladder of states with quantum number $K$ (angular momentum projected onto the symmetry axis). Each state decays quickly down the $K$-ladder to the lowest energy state $(J, K=J)$, e.g., $\mathrm{NH}_{3}(1,1)$. These states are metastable as they can only deexcite through forbidden transitions if the density is too low for collisional deexcitation and thus live long enough to be commonly observable in the ISM. The inversion lines are further split into hyperfine structure lines by the interaction of the electric quadrupole moment of the nitrogen atom with the electric field of the molecule's electrons, which allows the derivation of gas opacity from the ratio of hyperfine components. Fits of the entire line structure of at least two metastable ammonia lines thus yield opacity-corrected column density, line-of-sight velocity, line width, and rotational temperature. The rotational temperature is a lower limit and can be used to estimate the kinetic gas temperature up to approximately the line excitation energy via model-derived conversion functions.

Details on the ammonia thermometer, the derivation of opacity-corrected column density, rotational temperature, and its conversion into kinetic gas temperature are described in Appendix C.

\subsection{Hyperfine Structure Fitting in CLASS}

In order to derive gas temperatures without having to assume optically thin emission, we need to know line temperatures, line widths, and opacities. We derive these quantities by fitting the ammonia hyperfine structure with the $\operatorname{CLASS}^{26}$ package in GILDAS. $^{27}$ The $100^{\prime \prime}$ edge-masked data cubes were masked at $3 \sigma$ per channel and $5 \mathrm{Jy} \mathrm{beam}^{-1} \mathrm{~km} \mathrm{~s}^{-1}$ in integrated flux density to ensure sufficient $\mathrm{S} / \mathrm{N}$ for successful fits. Fitting is made with the CLASS functions method $\operatorname{nh} 3(j, j)$ and minimize for $J=1,2,3$. The higher transitions $J=4,5$, 6 are not implemented but can be fitted with the method hfs using the relative positions and strengths of the ammonia hyperfine structure components of Townes \& Schawlow (1975; listed in Table 3). The fitting algorithm is constrained to line opacities in the range $0.1 \leqslant \tau \leqslant 30$, which introduces a factor of

$$
\lim _{\tau \rightarrow 0} \frac{0.1}{\tau} \frac{1-e^{-\tau}}{1-e^{-0.1}}=1.051
$$

\footnotetext{
26 http://iram.fr/IRAMFR/GILDAS/doc/html/class-html/

27 https://www.iram.fr/IRAMFR/GILDAS/
} 

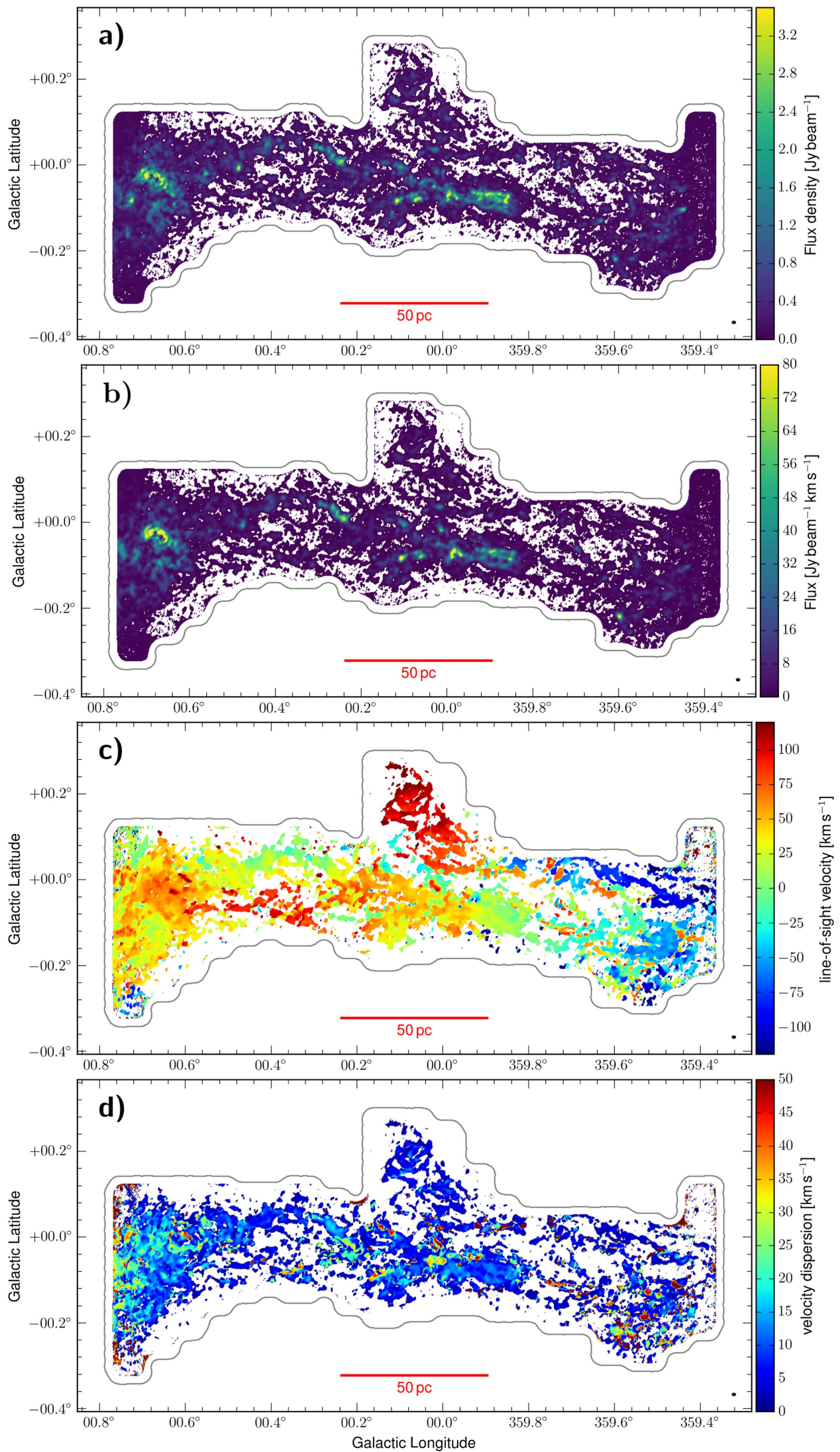

Figure 7. $\mathrm{NH}_{3}(3,3)$ moment maps: (a) peak intensity, (b) integrated intensity (moment 0 ), (c) intensity-weighted mean velocity (moment 1 ), and (d) intensityweighted velocity dispersion (moment 2). The intensity maps (a) and (b) are masked at $3 \sigma$, and velocity maps (c) and (d) are masked at $5 \sigma$ with an rms noise of $\sigma=13.1 \mathrm{mJy}$. The beam of $26 !{ }^{\prime \prime} 0 \times 17 !{ }^{\prime \prime}(1.05 \mathrm{pc} \times 0.71 \mathrm{pc})$ is indicated in the lower right-hand corner of each panel. 


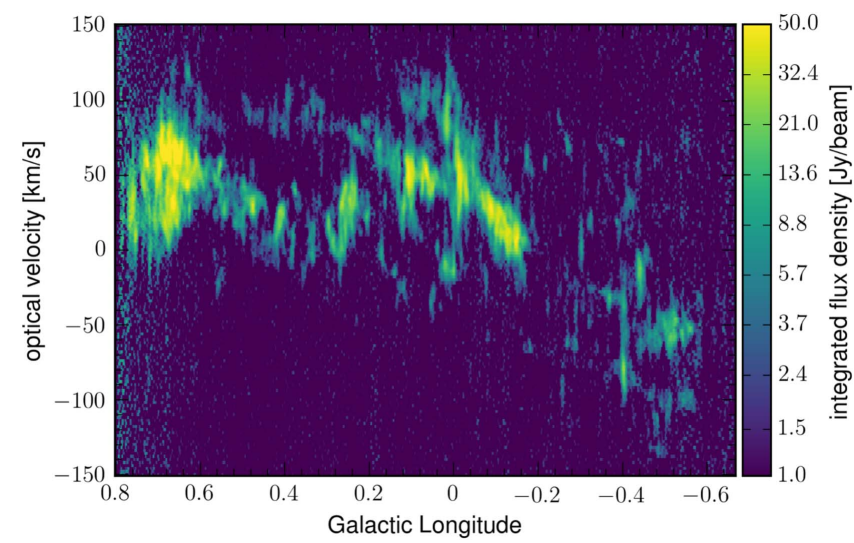

Figure 8. Position-velocity diagram along Galactic longitude, integrated over Galactic latitude.

relative to the line temperature of optically thin emission $(\tau \rightarrow 0)$. This deviation by up to $5.1 \%$ is small compared to the general flux density uncertainties of $\sim 15 \%$ (Figure 4 ) and can be neglected. The fit is further constrained to the strongest emission component along the line of sight. Occasionally, unsuccessful fits occur through blending of closely spaced emission components. These are discarded based on reduced $\chi^{2}$ (CLASS parameter lineRMS $>0.1)$, physically implausible values $(\Delta v(\mathrm{FWHM})>$ $\left.50.0 \mathrm{~km} \mathrm{~s}^{-1}\right)$, and the very large error of the fitted parameter $\left(\Delta\left(v_{\text {los }}\right)>10.0 \mathrm{~km} \mathrm{~s}^{-1}, \Delta(\Delta v)>10.0 \mathrm{~km} \mathrm{~s}^{-1}\right)$. Examples and statistics of the hyperfine structure fitting can be found in Appendix D.

\subsubsection{Sample Boltzmann Plot}

A Boltzmann plot (Appendix C.1, cf. Goldsmith \& Langer 1999), typical for the ammonia emission in the GC, is shown in Figure 9. The observed shapes do not follow a single linear relation, but become more shallow around $\mathrm{NH}_{3}$ $(3,3)$ to $\mathrm{NH}_{3}(4,4)$, which can be due to the nonlinear $T_{\text {kin }}-T_{\text {rot }}$ conversion or be indicative of a multiple temperature medium, as is already known in the literature (e.g., Hüttemeister et al. 1993; Mills \& Morris 2013). Errors in derived column density are typically small $\left(\Delta N_{\mathrm{u}} / N_{\mathrm{u}}\right.$ on the order of a few percent) but larger (a few tens of percent) at the edges of clouds because of the lower $\mathrm{S} / \mathrm{N}$, which allows the derivation of accurate temperatures of typically $5 \%-10 \%$ relative error $\left(T_{\text {rot }}\right)$ and $10 \%-25 \%$ after conversion into kinetic temperature. Additionally, the $\sim 15 \%$ flux density error (Figure 4) contributes to the column density errors.

\subsection{Results of the Hyperfine Structure Fitting}

As in Section 3, only $\mathrm{NH}_{3}(3,3)$ is shown to illustrate features common in all observed ammonia lines. As a representative temperature measure, we choose to show $T_{24}$ calculated from $\mathrm{NH}_{3}(2,2)$ and $\mathrm{NH}_{3}(4,4)$. The strongest line, $\mathrm{NH}_{3}(3,3)$, yields the temperature $T_{36}$, which suffers larger relative errors than $T_{24}$ because of the low intensity of the $\mathrm{NH}_{3}$ $(6,6)$ emission. We also calculate $T_{12}$ and $T_{45}$. Other combinations, like $T_{23}$, cannot be calculated because $\mathrm{NH}_{3}$ $(2,2)$ is a para state (one hydrogen nuclear spin antiparallel), while $\mathrm{NH}_{3}(3,3)$ is an ortho state (all hydrogen nuclear spins parallel). Generally, $\mathrm{NH}_{3}$ (i, i) with $i=3 n$ ( $n$ integer) are ortho,

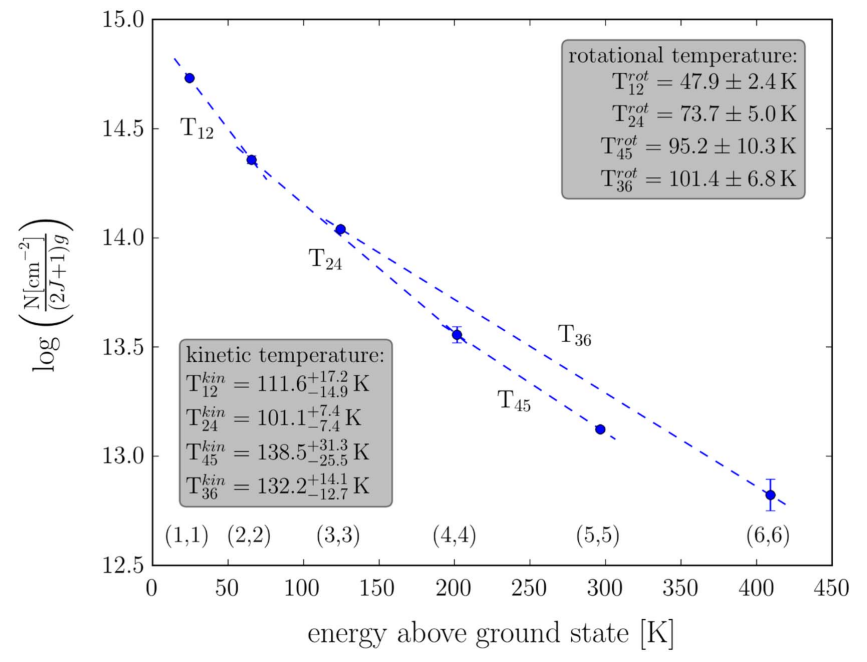

Figure 9. Typical Boltzmann plot (excitation state-scaled column density as a function of excitation temperature or energy above ground state) of ammonia emission in the Brick. Four temperature estimates (proportional to the inverse slope) are sketched and listed in the two boxes. Typical errors are 5\%-10\% in rotational and $10 \%-25 \%$ in kinetic temperature with increasing uncertainty for higher kinetic temperatures owing to the flattening of the $T_{\text {kin }}-T_{\text {rot }}$ conversion (Table 4; Morris et al. 1973; Ott et al. 2011; Gorski et al. 2017).

the others are para states. As the relative abundance of ortho versus para ammonia is not known, temperatures can only be estimated from two para states or two ortho states. For $T_{12}$, the $T_{\text {rot }}-T_{\text {kin }}$ conversion (Table 4 and Figure 5 in Ott et al. 2011; see also Morris et al. 1973) flattens, which prevents a reliable derivation of temperatures above $\sim 50-60 \mathrm{~K} . T_{24}, T_{45}$, and $T_{36}$ do not suffer such problems (Gorski et al. 2017) and yield very similar results. The corresponding maps for $T_{12}, T_{45}$, and $T_{36}$ can be found in Appendix E.

\subsubsection{Column Density}

The $\mathrm{NH}_{3}(3,3)$ column density $N_{l}$ (Figure 33) traces much of the ring-like gas stream by construction of the mask applied before hyperfine structure fitting. A peak of $N_{33}=7.7 \times 10^{15}$ $\mathrm{cm}^{-2}$ is reached in Sgr B2. Massive clouds like the Brick, $\mathrm{G}+0.10-0.08$, and the $20 \mathrm{~km} \mathrm{~s}^{-1}$ cloud are also found to have high column densities $\left(N_{33} \gtrsim 1.5 \times 10^{15} \mathrm{~cm}^{-2}\right)$, while $\mathrm{NH}_{3}$ $(3,3)$ is not largely excited and detected at column densities $\lesssim 1.5 \times 10^{15} \mathrm{~cm}^{-2}$. The $\mathrm{NH}_{3}(1,1)$ column density reaches a maximum of $1.2 \times 10^{16} \mathrm{~cm}^{-2}$ in $\operatorname{Sgr~B} 2$, where $\mathrm{NH}_{3}(6,6)$ is still detected at up to $2.6 \times 10^{15} \mathrm{~cm}^{-2}$.

The total ammonia column density as derived from Equation (22) (Figure 10) shows a similar picture as the $\mathrm{NH}_{3}$ $(3,3)$ column density. The highest column densities are reached in Sgr B2 $\left(6.8 \times 10^{16} \mathrm{~cm}^{-2}\right)$, the Brick $\left(3.9 \times 10^{16} \mathrm{~cm}^{-2}\right)$, and the $20 \mathrm{~km} \mathrm{~s}^{-1}$ cloud $\left(3.0 \times 10^{16} \mathrm{~cm}^{-2}\right)$, whereas most smaller clouds along the "ring" have $N_{\text {tot }} \lesssim 1.0 \times 10^{16} \mathrm{~cm}^{-2}$. The clouds at $l \sim 0.1, b \sim 0.2$ show the lowest detected column densities $\left(1.0 \times 10^{15} \lesssim N_{\text {tot }} \mathrm{cm}^{-2} \lesssim 2.5 \times 10^{15}\right)$ of clouds that do not fall below the fitting thresholds. Other clouds of this size typically exhibit column densities that are higher by a factor of 2-3. A small cloud of high column density is located at $l=-0.40, \quad b=-0.22$ with $N_{\text {tot }}$ up to $2.6 \times 10^{16} \mathrm{~cm}^{-2}$ $\left(N_{33}=6.4 \times 10^{15} \mathrm{~cm}^{-2}\right)$ and surrounded by gas of $N_{\text {tot }} \sim$ $10^{16} \mathrm{~cm}^{-2}$. This cloud is assumed to lie in the foreground (Longmore et al. 2013b) and is discussed to be influenced by an 

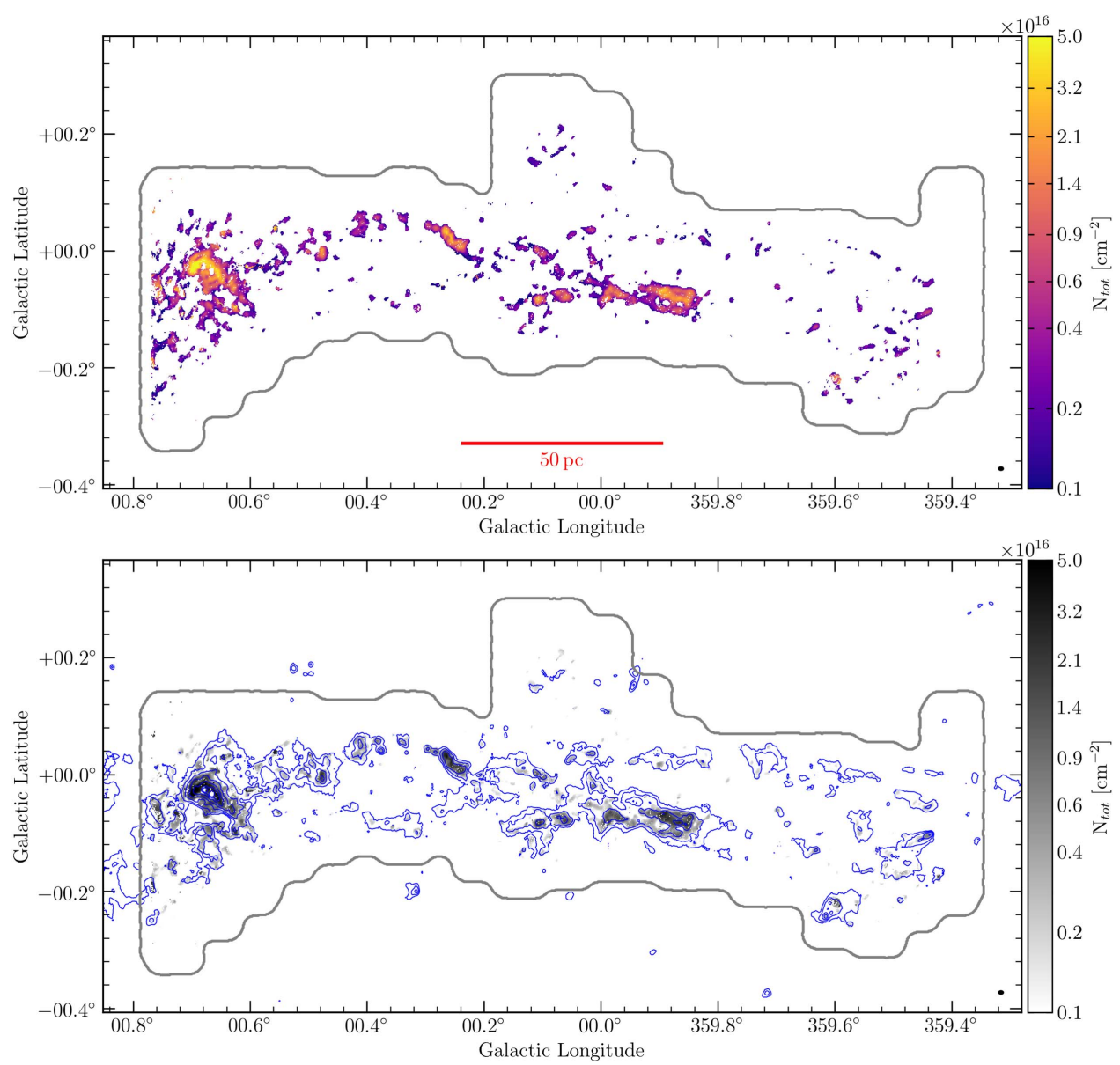

Figure 10. Top: total ammonia column density according to Equation (22). The dynamical range is $\sim 5 \times 10^{15} \mathrm{~cm}^{-2}$ to $\sim 6.8 \times 10^{16} \mathrm{~cm}^{-2}$. In warm clouds like Sgr B2, this figure represents a lower limit as the unobserved states $J>6$ can be populated in non-negligible fractions (Mills \& Morris 2013). Bottom: the same map of the total column density overlaid with $870 \mu \mathrm{m}$ dust emission from ATLASGAL (Schuller et al. 2009) at contours of powers of two $(1,2, \ldots, 64)$ Jy beam ${ }^{-1}$. The ammonia distribution closely follows dust emission in regions of high ammonia column density $\left(\gtrsim 1 \times 10^{16} \mathrm{~cm}^{-2}\right)$.

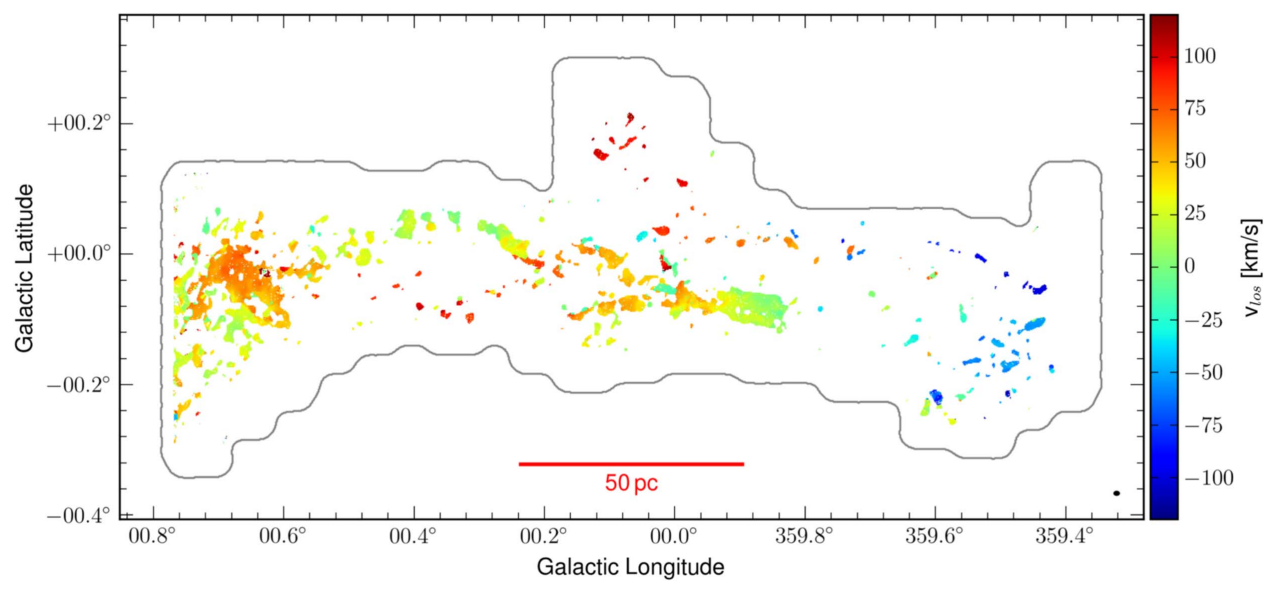

Figure 11. $\mathrm{NH}_{3}(3,3)$ line-of-sight velocity derived from fitting the hyperfine structure.

intermediate mass black hole (Oka et al. 2016) because of its unusually high $\mathrm{CO}$ velocity dispersion.

The ammonia distribution as traced by the $\mathrm{NH}_{3}$ column density map closely follows $870 \mu$ m dust emission obtained by ATLASGAL (Schuller et al. 2009), as can be seen in Figure 10 (bottom). The correlation is most pronounced for gas at higher column density than $\sim 1 \times 10^{16} \mathrm{~cm}^{-2}$. Lower column density gas is mostly found in regions of weak dust emission, but the presence of dust does not imply the detection of ammonia at $N_{\text {tot }} \gtrsim 2 \times 10^{15} \mathrm{~cm}^{-2}$. Almost all (dense) ammonia gas is accompanied by dust emission. Notable exceptions from the overall good correlation are found in Sgr B2 and Sgr A*. Sgr B2 (N) and Sgr B2 (M) are detected in absorption in ammonia, whereas the dust emission peaks at these locations. 


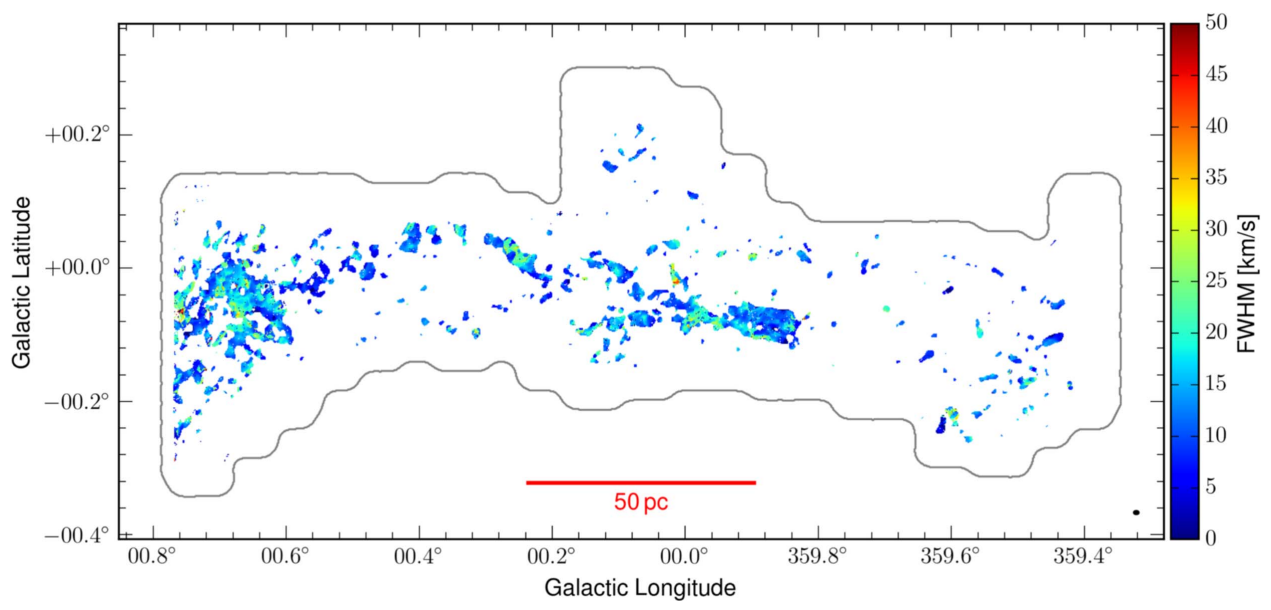

Figure 12. $\mathrm{NH}_{3}(3,3)$ line width (FWHM) derived from fitting the hyperfine structure.

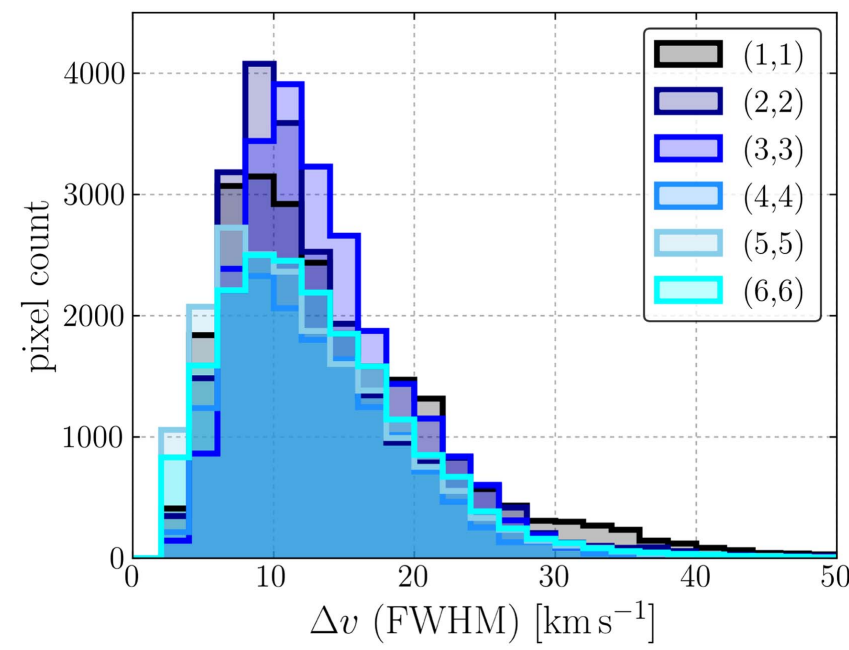

Figure 13. Distribution of FWHM of metastable ammonia hyperfine lines in the GC. The bin size of $2 \mathrm{~km} \mathrm{~s}^{-1}$ corresponds to the spectral resolution of the data cubes.

All of Sgr B2 that is detected in emission, however, does correlate very well with the $870 \mu \mathrm{m}$ contours. At Sgr A*, the $870 \mu \mathrm{m}$ emission locally peaks, but the ammonia column density is $\ll 1 \times 10^{15} \mathrm{~cm}^{-2}$ as this region falls below the selection thresholds for hyperfine structure fitting. The emission from $\mathrm{Sgr} \mathrm{A}^{*}$ at submm is contributed by optically thick synchrotron emission rather than thermal dust emission, and no correlation is observed.

\subsubsection{Kinematics}

The fit-derived line-of-sight velocity of $\mathrm{NH}_{3}(3,3)$, shown in Figure 11, generally agrees well with the moment-1 map (Figure 7(c)). Owing to the constraints for successful fitting (Section 4.1), the mapped area is considerably smaller. Significant differences between the two maps are not detected, which confirms that "successful" fits are indeed correct and potential "bad" fits were excluded.

The line widths of the fitted map (Figure 12) and the moment-2 map (Figure 7(d)) qualitatively agree about regions of higher or lower line width, but show very different quantitative results. After correcting for the conversion between FWHM (fitted map) and velocity dispersion $\sigma$ (moment map), a typical factor of $\sim 1.5-2$ (coresponding to differences of $<10 \mathrm{~km} \mathrm{~s}^{-1}$ ) remains, but extended regions all across the CMZ display factors of 3-5 (differences of $20 \mathrm{~km} \mathrm{~s}^{-1}$ up to $>50 \mathrm{~km} \mathrm{~s}^{-1}$ ). The larger line widths are derived from the moment map in all cases, meaning the image moment analysis overestimates the line width with respect to line fitting. This is partly due to unaccounted blending of hyperfine structure and mostly caused by multiple line-of-sight components in the moment map. Henshaw et al. (2016b) find similar differences between these two line width estimates in the CMZ based on HNCO data. Depending on the number of detected emission components along the line of sight, their median difference varies between $3.4 \mathrm{~km} \mathrm{~s}^{-1}$ (single component) and $18.8 \mathrm{~km} \mathrm{~s}^{-1}$ when four components are present. The maps derived from hyperfine structure fitting are thus considered to be more reliable than the simple moment analysis when investigating the kinematics, although fitting discards all but the strongest component along the line of sight, introducing a bias toward strong sources.

The distribution of fitted line widths for all ammonia transitions is shown in Figure 13 and shows a typical line width of $8-16 \mathrm{~km} \mathrm{~s}^{-1}$ consistently for all six observed ammonia lines. The peak of the distribution is located at $8-10 \mathrm{~km} \mathrm{~s}^{-1}$, and only $\mathrm{NH}_{3}$ (3, 3) has its peak at a slightly higher velocity of $10-12 \mathrm{~km} \mathrm{~s}^{-1}$.

\subsubsection{Opacity}

The opacity map of $\mathrm{NH}_{3}(3,3)$ (Figure 14) shows that a significant number of fitted pixels of the six ammonia species exhibit opacities $\tau \gtrsim 1$, where the often used assumption of optically thin emission does not hold anymore. This is the case not only in the very high column density clouds (like Sgr B2 and the Brick), but also in other dust ridge clouds and the inner regions of smaller clouds at negative longitudes and the far side of the "ring" $\left(l^{+}, b^{-}\right)$. A weak correlation between high column density (Figure 33) and high opacity (Figure 14) is found in the Brick, $\mathrm{G}+0.10+0.00$, the 20 and $50 \mathrm{~km} \mathrm{~s}^{-1}$ clouds, and the foreground cloud G-0.40-0.20. Other clouds are optically thin at low column density or do show high opacity despite having low column density $\left(N_{u, 33}<2.5 \times 10^{15} \mathrm{~cm}^{-2}\right.$, e.g., G0.50 +0.05 or G-0.60-0.09), which is indicative of a smaller filling fraction of clumpy gas. High opacities, especially in very small regions, must be taken with care as the edge of a region corresponds to a low $\mathrm{S} / \mathrm{N}$ ( $\gtrsim 3 \sigma$, see Section 4.1 for details), 


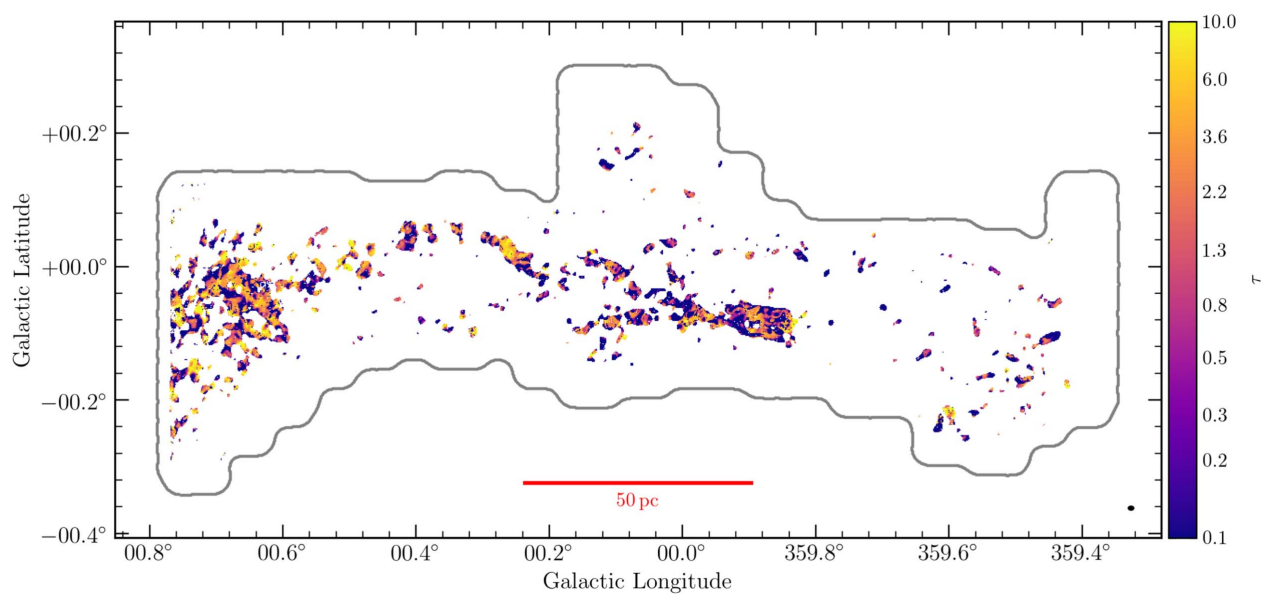

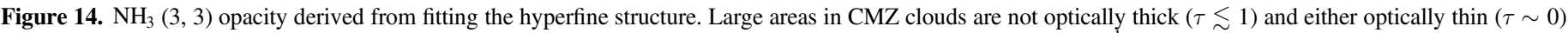
or marginally thick $(\tau<1)$. Several extreme exceptions of $\tau \gtrsim 10$ exist in the Brick, an extension to the $20 \mathrm{~km} \mathrm{~s} \mathrm{~s}^{-1}$ cloud, and the surrounding of Sgr B2.

and thus random noise peaks may be mistaken as hyperfine structure satellite components by the fitting routine. Because of the low flux of these pixels, the derived column density is only marginally affected, but opacity, which depends on the flux ratio of hyperfine structure components, can be artificially elevated. This opacity distribution is consistent with Hüttemeister et al. (1993), who observed $\mathrm{NH}_{3}(1,1),(2,2),(4,4)$, and $(5,5)$ in selected clouds in the GC. In the $(1,1)$ line, they find "strong" sources at $\tau_{11} \sim 4$ and an average $\bar{\tau}_{11}=2.3$ for "weak" sources, which matches our observations of $\bar{\tau}_{11}=2.0$ (median 1.2) and $\tau_{11} \gg 1$ in clouds when considering that SWAG also observed the less dense ISM in between clouds. We can also confirm the trend to lower opacity in Hüttemeister et al. (1993) for higher- $J$ lines as the median opacity decreases to the lower fitting limit $(\tau=0.1)$ for $\mathrm{NH}_{3}(4,4)$ and higher.

\subsubsection{Temperature}

The ammonia gas temperature as traced by kinetic temperature $T_{24}$, which is derived from $\mathrm{NH}_{3}(2,2)$ and $\mathrm{NH}_{3}(4,4)$, is shown in Figure 15. The typical temperature range observed in the GC is $50-120 \mathrm{~K}$, only Sgr B2 is considerably hotter with $\sim 200 \mathrm{~K}$.

A histogram of temperature measures $T_{12}, T_{24}, T_{45}$, and $T_{36}$ is shown in Figure 16 to illustrate the temperature distribution of the CMZ. $T_{12}$ cannot reliably trace temperatures $\gtrsim 50-60 \mathrm{~K}$ (see excitation energies listed in Table 1), and thus its distribution peaks at $\sim 35 \mathrm{~K}$, tracing cooler molecular gas. The other three temperature measures of higher lines are reliable to at least $\sim 200 \mathrm{~K}$ before the rotational-kinetic conversion starts to introduce large uncertainties, and they are therefore easier to interpret. Their distributions are similar in peak temperature and spread, with $T_{24}$ yielding higher values by $\sim 15 \mathrm{~K}$. Regarding the spatial distribution, most of the gas is found at intermediate temperatures at $60-100 \mathrm{~K}$ and in a few hot regions $T>150 \mathrm{~K}$.

The detection of two temperature components in the molecular gas is consistent with Hüttemeister et al. (1993), who found gas at rotational temperatures $T_{\text {rot }} \sim 25 \mathrm{~K}$ and $T_{\text {rot }} \sim 200 \mathrm{~K}$. Our cold $\left(T_{\text {kin }}=25-50 \mathrm{~K}\right)$ component is thus compatible with Hüttemeister et al. (1993) considering the rotational-kinetic temperature conversion. However, the temperature of the warm components differ significantly by a factor of $\sim 2-2.5$ in rotational temperature. Ao et al. (2013) and
Ginsburg et al. (2016) derived kinetic temperatures of dense molecular gas over large areas of the CMZ based on APEX observations of $\mathrm{H}_{2} \mathrm{CO}$ (formaldehyde). They find the gas to be warm at $50 \mathrm{~K}$ to $>100 \mathrm{~K}$ (Ao et al. 2013) and $\sim 60 \mathrm{~K}$ to $>100 \mathrm{~K}$ (Ginsburg et al. 2016), which is consistent with our detection of a warm gas component. Regarding the spatial distribution, the temperatures known in the literature match our results: Sgr B2 is hot at $\sim 200 \mathrm{~K}$ (this work), $>150 \mathrm{~K}$ (Ginsburg et al. 2016); the $20 \mathrm{~km} \mathrm{~s}^{-1}$ and $50 \mathrm{~km} \mathrm{~s}^{-1}$ cloud and the Brick show temperatures of $80-100 \mathrm{~K}$ (this work, Güsten et al. 1981; Mauersberger et al. 1986; Ao et al. 2013; Ginsburg et al. 2016).

We estimate the fraction of cold $(\sim 35 \mathrm{~K})$ and warm $(\sim 80 \mathrm{~K})$ gas of the total detected ammonia column density by fitting the Boltzmann plot (Figure 9) with two components. This allows the calculation of the column density of cold and warm ammonia independently and hence the relative abundances. Over the whole CMZ, we find the mean column density fractions to be $\sim 55 \%$ and $\sim 45 \%$ for the cold and warm component, respectively. In the 20 and $50 \mathrm{~km} \mathrm{~s}^{-1}$ clouds, the cold component dominates at 50\%-70\% (mean: 68\%) the relative contribution to the observed ammonia column density. In Sgr B2, a larger fraction of the column density is in the warm component, which results in both components being almost equally strong ( $\sim 50 \%$ cold and warm). Mean values of $\sim 60 \%$ of the cold gas fraction are found in the dust ridge and $~ 53 \%$ in clouds at negative longitudes (excluding the $20 \mathrm{~km} \mathrm{~s}^{-1}$ and $50 \mathrm{~km} \mathrm{~s}^{-1}$ clouds). The "Three Little Pigs" (G+0.05-0.07 "sticks," G+0.10-0.08 "stones," and G+0.15-0.09 "straw") are a group of clouds of similar global properties but very different substructure. They are also striking in the cold gas fraction $f_{\text {cold }}$ as $\mathrm{G}+0.10-0.08$ ("sticks") shows the highest $f_{\text {cold }}$ over an extended region at $\sim 81 \%$, whereas its neighbor $\mathrm{G}+0.05-0.07$ ("stones"; $f_{\text {cold }} \sim 65 \%$ ) seems inconspicuous and much closer to the CMZ mean. G+0.15-0.09, "straw," does not show up as its ammonia emission was to low to be hyperfine structure fitted. These results show that the typical gas temperature is not the same over the whole CMZ, but is either $\sim 35 \mathrm{~K}$ or $\sim 80 \mathrm{~K}$ depending on the considered region.

The relation between Hi-GAL dust temperature (Molinari et al. 2011) and ammonia gas temperature is shown in Figure 17 for $T_{24}$. Further tracers are shown in Appendix F. $T_{24}$ is offset well above the one-to-one relation by a factor of $\sim 3$ to $>5$. A linear best fit, calculated in bins of $1 \mathrm{~K}$ for 


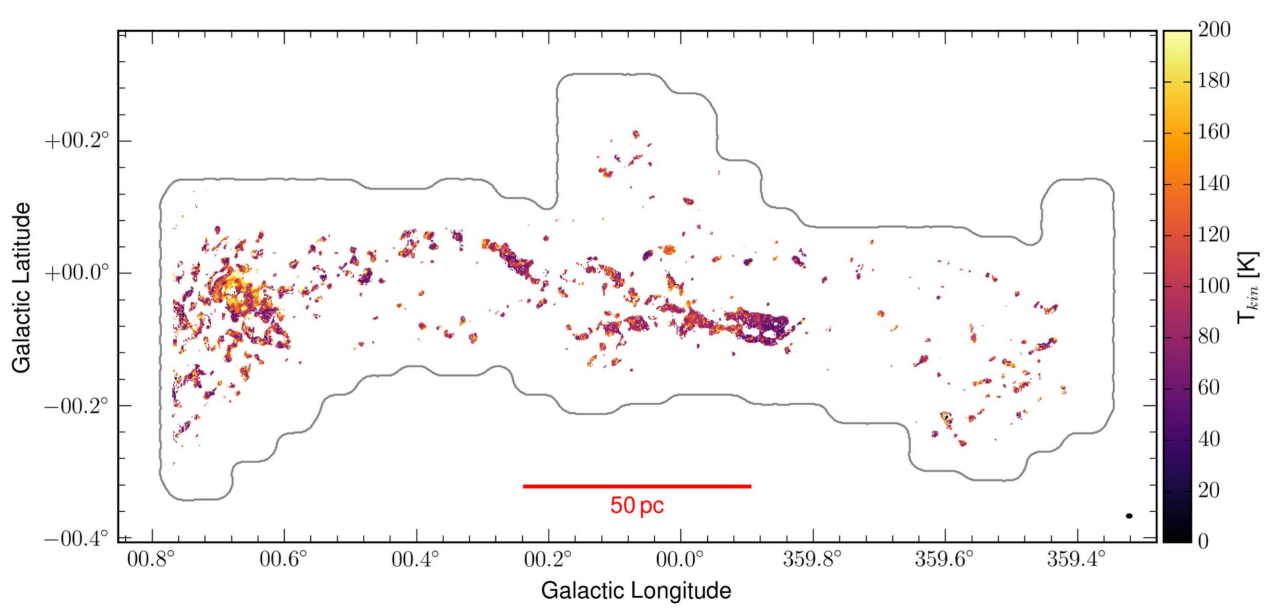

Figure 15. Kinetic ammonia gas temperature map $\left(T_{24}\right)$ derived from $\mathrm{NH}_{3}(2,2)$ and $\mathrm{NH}_{3}(4,4)$ hyperfine structure fits.

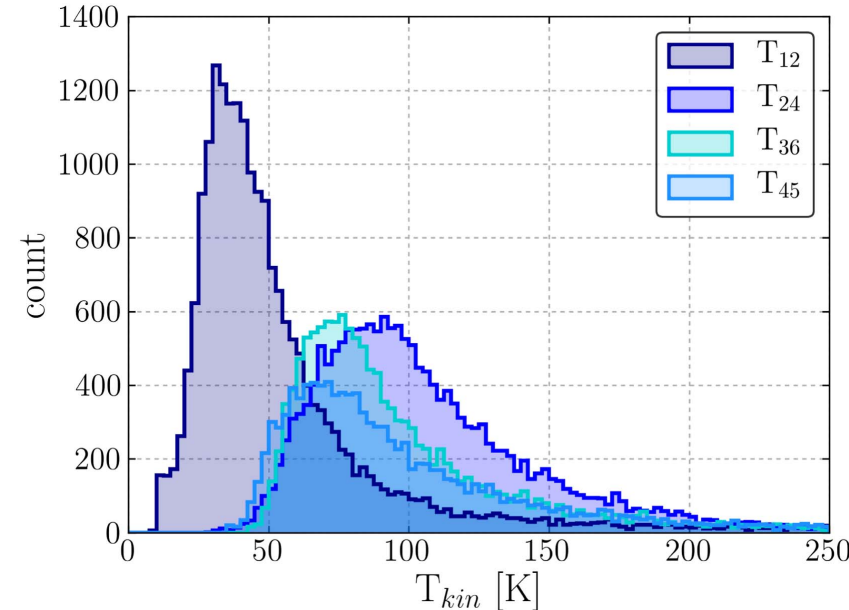

Figure 16. Distribution of temperatures in the GC as derived for four kinetic ammonia gas temperatures. The conversion of rotational to kinetic temperature is taken from Ott et al. (2011), as described in Section C. $T_{i j}$ denotes the temperature measure derived from the lines $\mathrm{NH}_{3}(\mathrm{i}, \mathrm{i})$ and $\mathrm{NH}_{3}(\mathrm{j}, \mathrm{j}) . T_{12}$ is sensitive to cold gas, whereas higher lines need warmer conditions to be excited and are thus succeptible only to increasingly warm gas. $T_{24}, T_{45}$, and $T_{36}$ trace similarly warm gas of $>50 \mathrm{~K}$.

$18 \mathrm{~K} \leqslant T_{\text {dust }} \leqslant 30 \mathrm{~K}$, yields a gradient of $d T_{24} / d T_{\text {dust }}=1.8$, and fitting without binning results in no correlation (gradient $\sim 0.0$ ) because of cutoff effects of the limited temperature reliability range. This result implies that in the CMZ, the same heating mechanism is likely to be responsible for both dust and gas heating although their temperatures are not coupled to the same value. This hint toward a common heating source contradicts the prediction by Clark et al. (2013), who explain the temperature structure of the Brick $(\mathrm{G} 0.253+0.016)$ by independent heating of gas and dust by cosmic rays and an interstellar radiation field, respectively. The observed correlation is compatible with heating by turbulence on large scales and compression on cloud scales (cf. Section 5), as was also suggested by Ginsburg et al. (2016). The fact that dust acts as a coolant in the CMZ has been known in the literature for a long time (e.g., Güsten et al. 1981; Molinari et al. 2011; Ginsburg et al. 2016) and can be confirmed by our new data. Similar results are obtained for warm molecular gas traced by $T_{45}$ and $T_{36}$ $\left(d T_{45} / d T_{\text {dust }}=2.4, d T_{36} / d T_{\text {dust }}=1.4\right)$. The cold molecular gas component $\left(T_{12}\right)$ only weakly correlates with dust temperatures

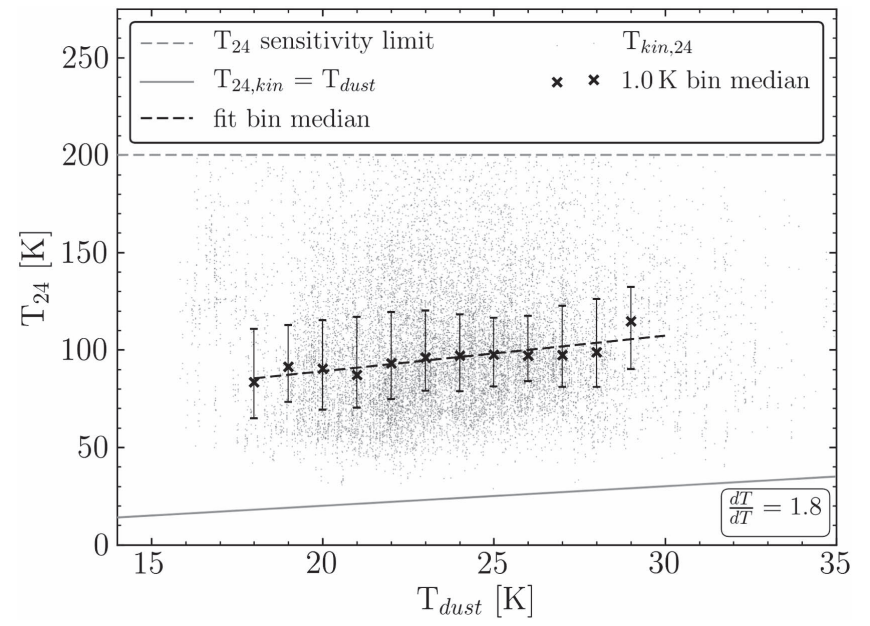

Figure 17. Correlation of Hi-Gal dust temperature (Molinari et al. 2011) and kinetic ammonia gas temperature $T_{24}$. Black crosses denote median $T_{24}$ temperature in bins of $1.0 \mathrm{~K}$ dust temperature, which are linearly fitted (black dashed line). The errors are the range that includes $50 \%$ of measurements in the respective bin. Median temperatures are correlated despite the offset relative to $T_{24}=T_{\text {dust }}$.

$\left(d T_{12} / d T_{\text {dust }}=0.5\right)$, which implies a temperature component that is not reflected by dust measurements.

\subsubsection{Estimation of the Beam Filling Factor}

A simple estimation of the beam filling factor $\eta_{f}$ can be made based on the comparison of $T_{\text {kin }}$ and brightness temperature $T_{b}$. For $\eta_{f}=1$, both temperatures must be equal, but if the beam is partly covered by emission of temperature $T_{\text {kin }}$ and partly by weak (i.e., cold) background, the observed $T_{b}$ is lower. A firstorder estimate is given by $\eta_{f}=T_{b} / T_{\text {kin }}$ under the assumptions of $\eta_{f}$ being identical for the two lines $\mathrm{NH}_{3}(\mathrm{i}, \mathrm{i})$ and $\mathrm{NH}_{3}(\mathrm{j}, \mathrm{j})$ that define temperature $T_{i j}$. This furthermore assumes high optical depth and the line excitation temperatures being approximately given by $T_{\text {kin }}$. Typical values of dense clouds (the $20 \mathrm{~km} \mathrm{~s}^{-1}$ cloud, the Brick, cloud d, Sgr B2) are in the range $0.10-0.15$, Sgr $\mathrm{C}$ and less dense clouds are covered at $\eta_{f}=0.05-0.10$ (e.g., the "Three Little Pigs" G0.05-0.07, G0.10-0.08, and G0.15-0.09). G-0.40-0.25 sticks out from all other clouds at negative longitudes with $\eta_{f} \sim 0.10$, which is the third highest $\eta_{f}$ observed after Sgr B2 and the $20 \mathrm{~km} \mathrm{~s}^{-1}$ 


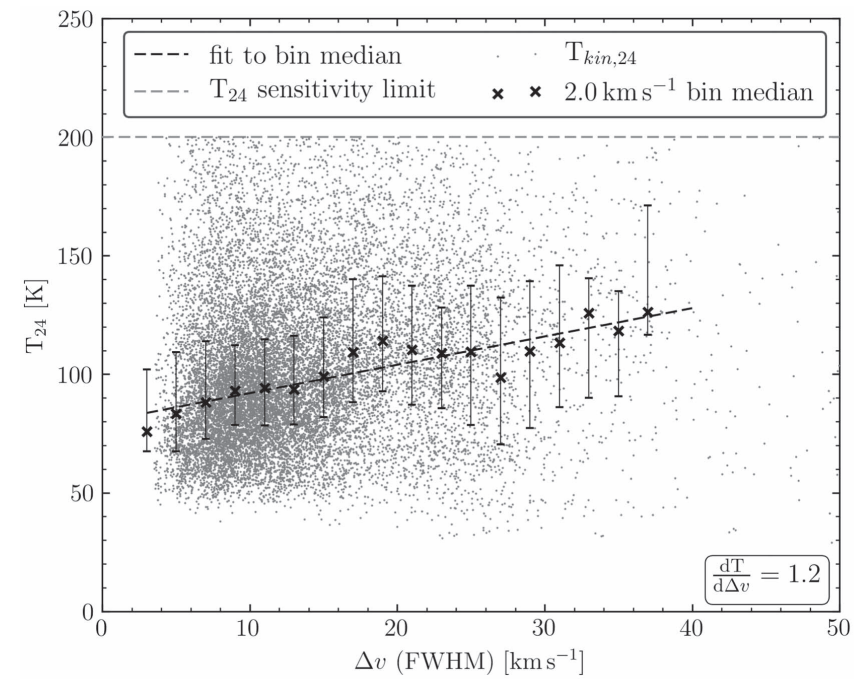

Figure 18. Kinetic ammonia temperature $T_{24}$ as a function of line width of $\mathrm{NH}_{3}$ $(2,2)$. Median temperatures in bins of $2.0 \mathrm{~km} \mathrm{~s}^{-1}$ width are marked by black crosses and fitted linearly. The errors are the range that includes $50 \%$ of measurements in the respective bin. A cutoff at $200 \mathrm{~K}$ is applied, above which the conversion of rotational into kinetic temperature becomes increasingly unreliable (Table 4, Morris et al. 1973; Ott et al. 2011; Gorski et al. 2017).
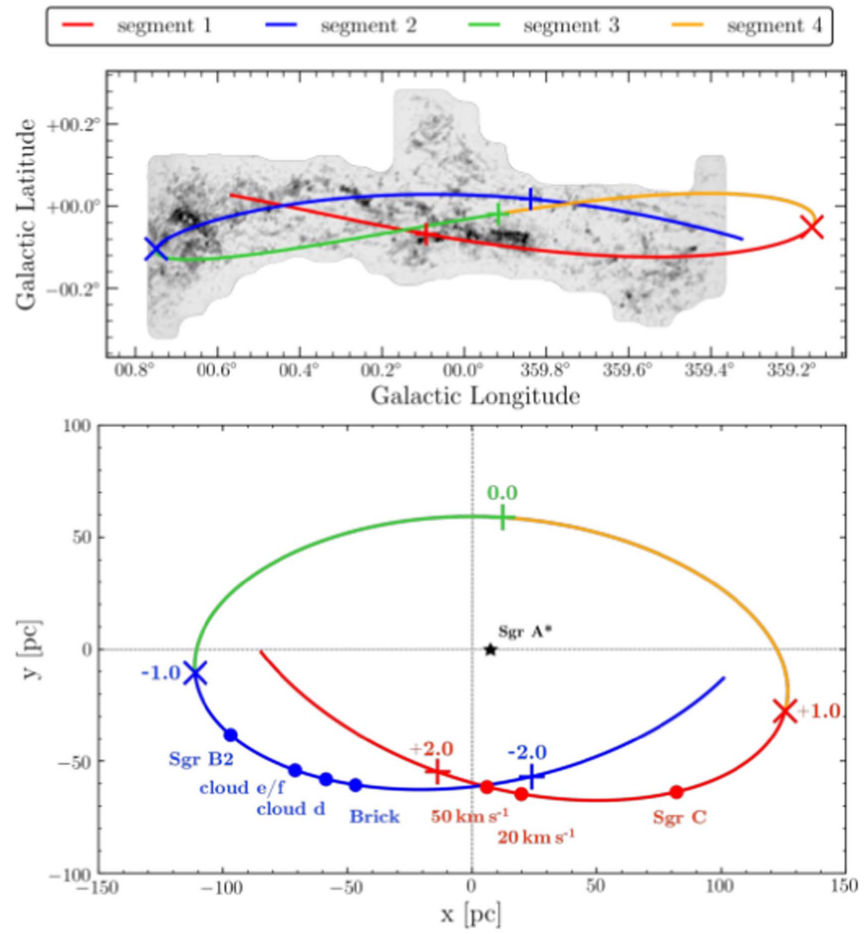

Figure 19. Overview of the stream orbit according to K15. Pericenters are marked by plus symbols, crosses denote apocenters. The bottom of the gravitational potential at Sgr A* is marked by a star. Top: projected view of the stream orbit in Galactic coordinates. Background image: SWAG $\mathrm{NH}_{3}(3,3)$ integrated intensity. Bottom: top-down view in physical coordinates. The observer is situated in negative $y$-direction. Major molecular clouds are labeled. Time since far-side pericenter passage is given for peri- and apocenters in Myr.

cloud. All other clouds show beam filling factors below 0.05 . The average size of cloud substructure (clump size) for $\eta_{f}=0.10(0.050 .15)$ is then $0.27 \mathrm{pc}(0.19 \mathrm{pc}, 0.33 \mathrm{pc})$. This is consistent with recent interferometer measurements with the SMA and ALMA (Kauffmann et al. 2013, 2017a; Rathborne et al. 2014b; Lu et al. 2017).

\subsubsection{Correlation of Fitted Parameters}

Figure 18 shows the relation between line width and temperature for $T_{24}$ and the corresponding line $\mathrm{NH}_{3}(2,2)$. As before, temperatures scatter between $50 \mathrm{~K}$ and the sensitivity cutoff at $200 \mathrm{~K}$ for line widths of $\sim 5 \mathrm{~km} \mathrm{~s}^{-1}$ to $30-40 \mathrm{~km} \mathrm{~s}^{-1}$ with no obvious correlation. Temperature medians in bins of $2 \mathrm{~km} \mathrm{~s}^{-1}$ width, however, follow a linear relation at $d T / d(\Delta v)=1.2$. The increase in line width due to thermal broadening scales sub-linearly as $\Delta v \propto \sqrt{T}$ and is about one order of magnitude $\left(\Delta v_{\text {thermal }} \sim 2 \mathrm{~km} \mathrm{~s}^{-1}\right)$ weaker than the observed linear relation. Assuming that the line width is not contaminated by blending of clouds along the line of sight, this implies that on a statistical basis, the more turbulent gas is warmer but large variations between clouds do exist, as has also previously been detected by Immer et al. (2016) and Ginsburg et al. (2016).

\section{Discussion}

We now compare the observations to models proposed in the literature with a focus on the stream model by K15, as introduced in Section 1. In practice, this means that positions on the sky are mapped to times and examined for consistent sequential behavior. Figure 19 offers an overview of the orbit's 3D structure, of the relation to major molecular clouds, and of evolutionary timescales.

\subsection{Orbital Fitting and Statistical Analysis}

The mapping of cloud positions to times in the K15 model is made in PPV space. Within $\pm 20 \mathrm{~km} \mathrm{~s}^{-1}$ in line-of-sight velocity and 0.15 projected distance, a cloud is matched to the nearest point along the orbit for which time and other orbital parameters are known. The line-of-sight velocity of clouds is given by the mean velocity derived from the $\mathrm{NH}_{3}$ hyperfine structure fits (Section 4.2.2). Clouds farther from the stream than 0.15 in the Galactic longitude/latitude plane are considered to not belong to the stream as this is approximately the maximum spatial separation found by K15 (see their Figure 4). Out of the 24383 fitted pixels, we match 19453 pixels to the stream (79.8\%), while 4930 pixels (20.2\%) contain emission from clouds that are not related to the gas stream. $^{28}$

We examine the kinetic ammonia temperatures $T_{24}, T_{45}$, and $T_{36}$, as well as column density and line width of the six metastable inversion lines covered by SWAG along the stream. Medians are calculated in bins of $0.01 \mathrm{Myr}$ if the bin contains more than 10 members, and therefore the sample size is large enough for the median to be considered robust against outliers. Errors to the medians are given by the range that includes the central $50 \%$ of all measurements in the respective bin. The resulting time dependencies of physical parameters are assessed by fitting linear functions to the medians if a trend is apparent.

\subsection{Time Dependencies}

The time dependencies of kinetic temperature $T_{24}$, column density, and line width of $\mathrm{NH}_{3}(3,3)$ are shown in the following sections to represent results that appear in all of the ammonia temperature measures $\left(T_{24}, T_{45}, T_{36}\right)$ or ammonia inversion lines $(J=1, \ldots, 6)$ if not explicitly noted otherwise. Plots of the other

\footnotetext{
28 This gas is mostly located on the polar spur at $l \sim 0$ and in the surrounding of Sgr B2 and thus too far $(>0.15)$ from the stream.
} 


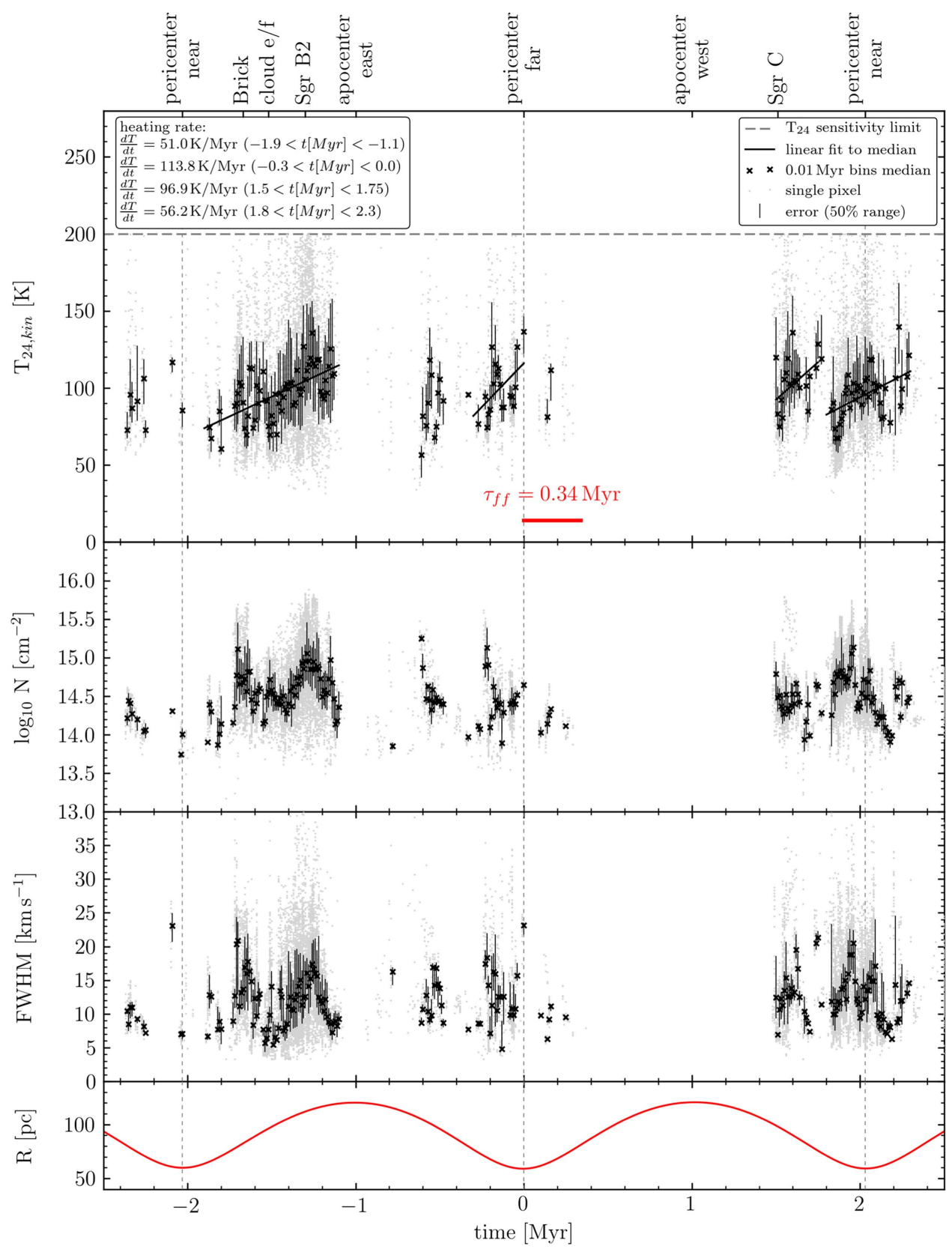

Figure 20. Kinetic ammonia temperature $T_{24}$ (top), $\mathrm{NH}_{3}(3,3)$ column density (middle top), and $\mathrm{NH}_{3}(3,3)$ line width (middle bottom) as a function of time since farside pericenter passage (bottom $x$-axis, top $x$-axis shows important dynamical points in the K15 model and massive molecular clouds). The bottom panel shows the radial distance from the center of the Galactic potential. Measurements for individual pixels (light gray points) are overlaid with black crosses denoting medians in bins of $0.01 \mathrm{Myr}$ and error intervals that include $50 \%$ of the data in each bin. Linear fits to the median temperature and corresponding heating rates are shown for four regions of consistent time evolution.

temperature measures and ammonia lines can be found in Appendix G. The origin of the timescale in plots versus time is chosen to match K15 and as such identifies $0.0 \mathrm{Myr}$ $( \pm 2.03 \mathrm{Myr})$ with far (near) side pericenter passages, respectively (as labeled in Figure 19).

Although ammonia hyperfine structure fits were derived at the projections of all four stream segments, several time ranges are not well sampled. Especially, $0.3 \lesssim t[\mathrm{Myr}] \lesssim 1.5$ is not covered. This time range corresponds to stream segment 4 , where little gas is found because of the large-scale $1^{+} / 1^{-}$ asymmetry of the CMZ. The time ranges most interesting to this study, the dust ridge and around the pericenter passages at $0 \mathrm{Myr}$ and $+2 \mathrm{Myr}$, are well sampled.
The potential evolution can be fit in up to four regions of significant data density: in the dust ridge, -1.90 $<t[\mathrm{Myr}]<-1.1$, at the far-side pericenter passage, -0.30 $<t$ [Myr] $<0.05$, at the near-side pericenter passage, at $1.75<t[\mathrm{Myr}]<2.25$, and in clouds near Sgr C, 1.50 $<t[\mathrm{Myr}]<1.75$.

\subsubsection{Gas Temperature}

The top panel of Figure 20 shows the kinetic ammonia temperature $T_{24}$ as a function of time. Median kinetic temperatures of $\sim 55 \mathrm{~K}$ to $\sim 135 \mathrm{~K}$ are detected with typical error margins of $[-11 \mathrm{~K},+15 \mathrm{~K}]$. Four sequences of consistently increasing temperature are found in the time intervals $[-1.9 \mathrm{Myr}$, 
Table 2

Ammonia Kinetic Temperature Gradients (Heating Rates) of the Four Recovered Temporal Sequences (Figure 20) and of the Orbital Phase without and with Adjusting the Zero-point (Figure 21)

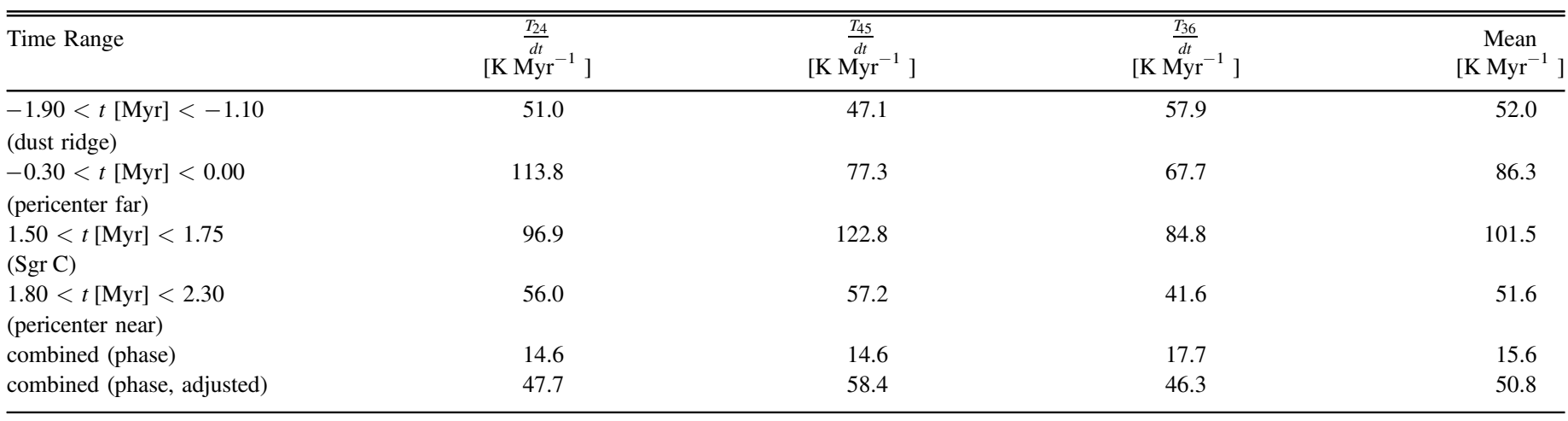

Note. Errors are $\sim 100 \%$ for individual heating rates and $\sim 58 \%$ for the mean.

$-1.1 \mathrm{Myr}], \quad[-0.3 \mathrm{Myr}, 0.05 \mathrm{Myr}], \quad[1.5 \mathrm{Myr}, 1.8 \mathrm{Myr}]$, and [1.8 Myr, 2.3 Myr]. Positive linear temperature gradients of 51 and $56 \mathrm{~K} \mathrm{Myr}^{-1}$ are derived in the dust ridge and around the near-side pericenter, whereas steeper gradients of $114 \mathrm{~K} \mathrm{Myr}^{-1}$ at pericenter far and $97 \mathrm{~K} \mathrm{Myr}^{-1}$ at pericenter near are fitted. Owing to the short sampled time range and the few temperature measurements, the sequence at pericenter far must be taken with caution. The error intervals are large because of the chosen conservative error estimation (Section 5.2), which causes the heating rate errors derived from $\chi^{2}$-fitting to also show a substantial error of typically $\sim 100 \%$, which is likely overestimated given the consistent trend. The mean heating rate of the three individual measurements $\left(T_{24}, T_{45}, T_{36}\right)$ thus has a relative error of approximately $58 \%$.

In the dust ridge (Brick to Sgr B2, $-1.9<t$ [Myr] $<-1.1$ ), the covered time range $(0.8 \mathrm{Myr})$ and statistics $(\sim 9200$ measurements) yield reliable heating rates of $47.1 \mathrm{~K} \mathrm{Myr}^{-1}$ to $57.9 \mathrm{~K} \mathrm{Myr}^{-1}$ (mean of $52.0 \mathrm{~K} \mathrm{Myr}^{-1}$ ) in the three partly independent temperature measures $T_{24}, T_{45}$, and $T_{36}$ (Table. 2). The absolute increase is from $75 \mathrm{~K}$ to $110 \mathrm{~K}\left(T_{24}\right), 60 \mathrm{~K}$ to $105 \mathrm{~K}\left(T_{45}\right)$, and $60 \mathrm{~K}$ to $100 \mathrm{~K}\left(T_{36}\right)$ or $\sim 40 \mathrm{~K}$ on average.

The short sequence at the far-side pericenter passage $(-0.30<t[\mathrm{Myr}]<0.00)$ is affected by low number statistics and has heating rate errors larger than $100 \%$. Any conclusion for this time range should thus be treated merely as a qualitative but not as a quantitative result.

Between the western apocenter of the orbit and the pericenter on the near side, two independent sequences of increasing temperature are found, separated by a discontinuity at $1.8 \mathrm{Myr}$, where the median temperature sharply drops from $\sim 120 \mathrm{~K}$ to $\sim 85 \mathrm{~K}$. This is again seen at a very similar level in $T_{24}, T_{45}$ and $T_{36}$. Heating rates differ between the sequence at $\mathrm{Sgr} \mathrm{C}$ and pericenter, the former being consistently higher at $\sim 102 \mathrm{~K} \mathrm{Myr}^{-1}$ than the latter $\left(\sim 52 \mathrm{~K} \mathrm{Myr}^{-1}\right.$, mean heating rates).

An overview of derived heating rates in the four sequences is given in Table 2.

\subsubsection{Column Density}

The median column densities show no evidence of time dependence (Figure 20, middle top, Appendix G) in the dust ridge. No consistent trend is apparent as column densities increase strongly in the Brick and Sgr B2 above a flat or, at most, very weakly rising base level. These clouds are already known to be denser than other dust ridge clouds and thus are expected to be more massive outliers to any potential underlying relation (Lis \& Carlstrom 1994; Lis et al. 1994; Molinari et al. 2011; Longmore et al. 2012; Pillai et al. 2015). Given that the column densities in between the Brick and Sgr B2 show no rise and also drop to the same level after Sgr B2, we conclude that there is no evolutionary column density sequence in the dust ridge.

The clouds around near-side pericenter passage show weak evidence for decreasing column densities where the median column density drops for $J=1,2,3$ but can be considered constant within the scatter for $J=4,5$, and 6 . This relation is likely also influenced by two individual objects, the $20 \mathrm{~km} \mathrm{~s}^{-1}$ and $50 \mathrm{~km} \mathrm{~s}^{-1}$ clouds, although density bumps are not as prominent as in Sgr B2.

At far-side pericenter, medians scatter by up to $\sim 1$ dex and no conclusion can be drawn. The column densities in the Sgr C region are constant for all six ammonia tracers, with very low scatter compared to the rest of the covered time ranges.

Qualitatively and quantitatively very similar results are derived for opacity due to the correlation of opacity and column density, and this is hence not discussed further.

\subsubsection{Line Width}

The evolution of the median line width (FWHM) is similar to that of the column density, but shows an even less consistent behavior, as can be seen in Figure 20 (middle bottom) for $\mathrm{NH}_{3}$ $(3,3)(J=1, \ldots, 6$ in Appendix G). In the dust ridge, line widths vary by a factor of $\sim 2.5$ with peaks in the Brick and Sgr B2. There is no sign that these could be just outliers to an underlying trend as line widths are at similar levels of 6-10 $\mathrm{km} \mathrm{s}^{-1}$ before the Brick, in between Brick and Sgr B2, and after Sgr B2. Any potential time dependency of the line width thus cannot be stronger than a few $\mathrm{km} \mathrm{s}^{-1} \mathrm{Myr}^{-1}$ to be hidden within the observed scatter. Results are very similar for the other ammonia lines $(J=1,2,4,5,6)$ at slightly varying absolute level, as could already be seen in the line width histogram (Figure 13).

In the other time ranges that show temperature evolution, no sign of consistent line width trend can be found. As for column density, the 20 and $50 \mathrm{~km} \mathrm{~s}^{-1}$ clouds are weak outliers toward higher values.

The increase in line width at the position of large and massive clouds can be caused by scaling relations ("Larson 


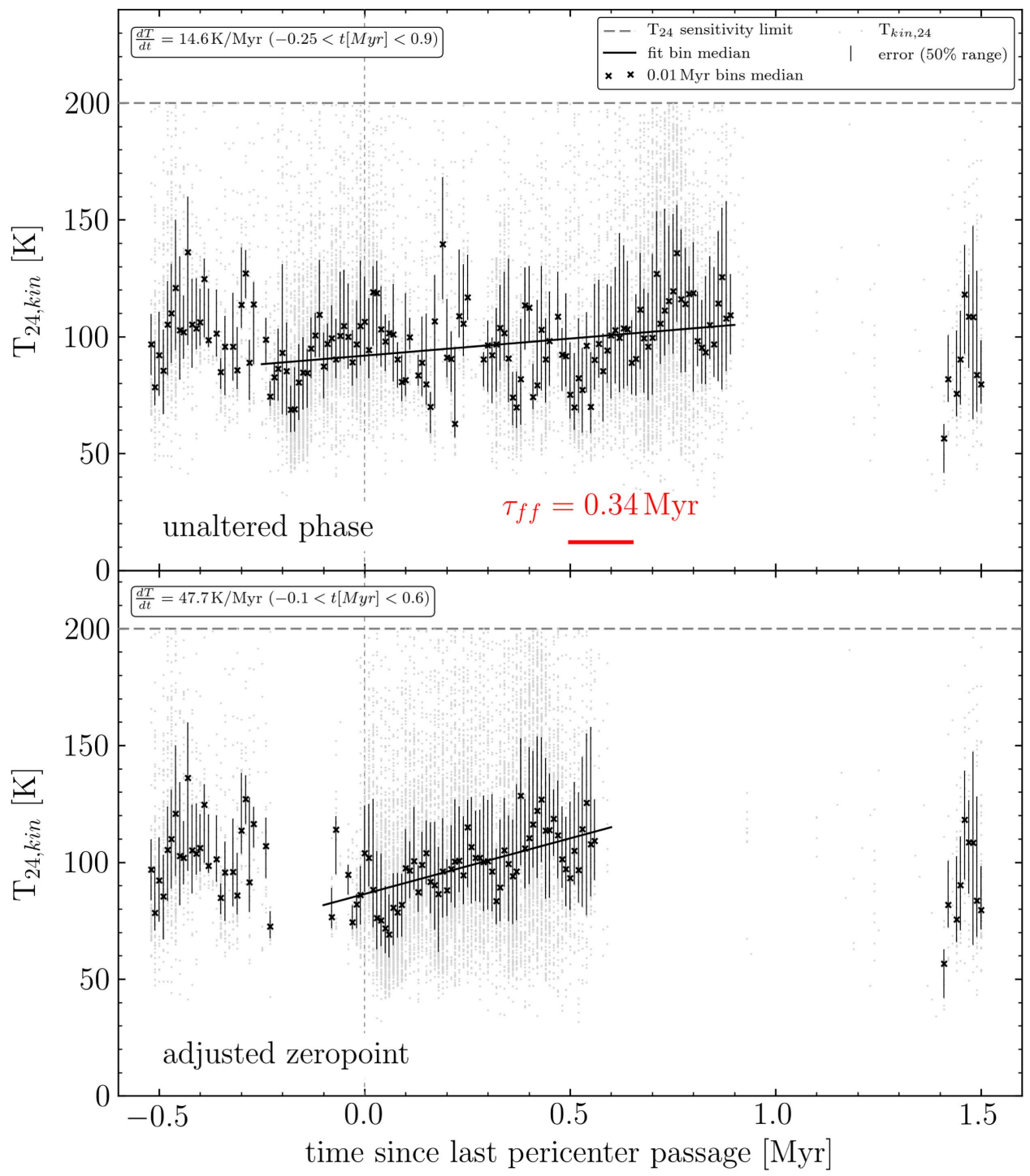

Figure 21. Kinetic ammonia temperature $T_{24}$ as a function of time since the last occurrence of pericenter passage (orbital phase). Symbols and error bars are the same as in Figure 20. The top panel shows the unaltered data as in Figure 20 (top panel), whereas in the bottom panel, the zero-point of the sequences in the dust ridge, pericenter near, and pericenter far are adjusted to maximize the heating rate. Within a range of reasonable offsets in zero-point of $\pm 0.3 \mathrm{Myr}$, the best fit is achieved for $-0.3 \mathrm{Myr}$ (dust ridge), $+0.2 \mathrm{Myr}$ (pericenter near), and $+0.2 \mathrm{Myr}$ (pericenter far). The fitted time ranges of $-0.25 \mathrm{Myr}$ to $+0.9 \mathrm{Myr}$ and $-0.1 \mathrm{Myr}$ to $0.6 \mathrm{Myr}$ result from the sum of the fits in the time domain.

laws," Larson 1981), be an effect of hyperfine structure fitting, or an effect of projection. CMZ clouds are known to follow a steep scaling relation of line width $\sigma$ with cloud radius $R$ : $\sigma \propto R^{0.6-0.7}$ (Shetty et al. 2012; Kruijssen \& Longmore 2013; Kauffmann et al. 2017b), and thus line widths are expected to increase by a factor of $\sim 1.5$ between the small clouds $\mathrm{b} / \mathrm{c}$ and larger clouds like the Brick and cloud e/f (Immer et al. 2012). The observed variation in line width of a factor $\sim 2.5$ is thus only partly due to variations in cloud size and other sources of scatter must be present, such as unresolved blending of multiple emission components along the line of sight, as shown in Figure 36(d)). Spectra with two close components with separations of a few to a few tens of $\mathrm{km} \mathrm{s}^{-1}$ are more likely to be present in larger clouds and thus cause the same signature as the scaling relation does, increasing the overall scatter. Projection effects may also increase the apparent line width through pile-up along the line of sight at the tangent points of the orbit. This can play a role in Sgr B2, but is not expected to affect the other clouds (cf. Figure 19).

\subsection{Discussion in the Context of the Stream Model and Tidal Triggering}

As shown above, sequences of increasing temperature are found, but no such sign is recovered for line width and column density. At near-side pericenter passage, an anticorrelation of increasing temperature and potentially decreasing column density is found, whereas the positive-temperature gradients in the dust ridge at far-side pericenter and around $\mathrm{Sgr} \mathrm{C}$ do not have a detectable counterpart in column density or line width. Based on the concept of tidally triggered SF (Longmore et al. 2013b), we discuss the evolution at and after pericenter passage separately in Sections 5.3.1 and 5.3.2. By the time-tagging method used in this work, the interpretation is furthermore linked to the stream model of K15, but general conclusions can be transferred to other orbital models. If a model includes radial oscillation, it can trigger cloud collapse and may give rise to a sequence of star formation. If the clouds were on circular orbits, our time-tagging method would still result in accurate 


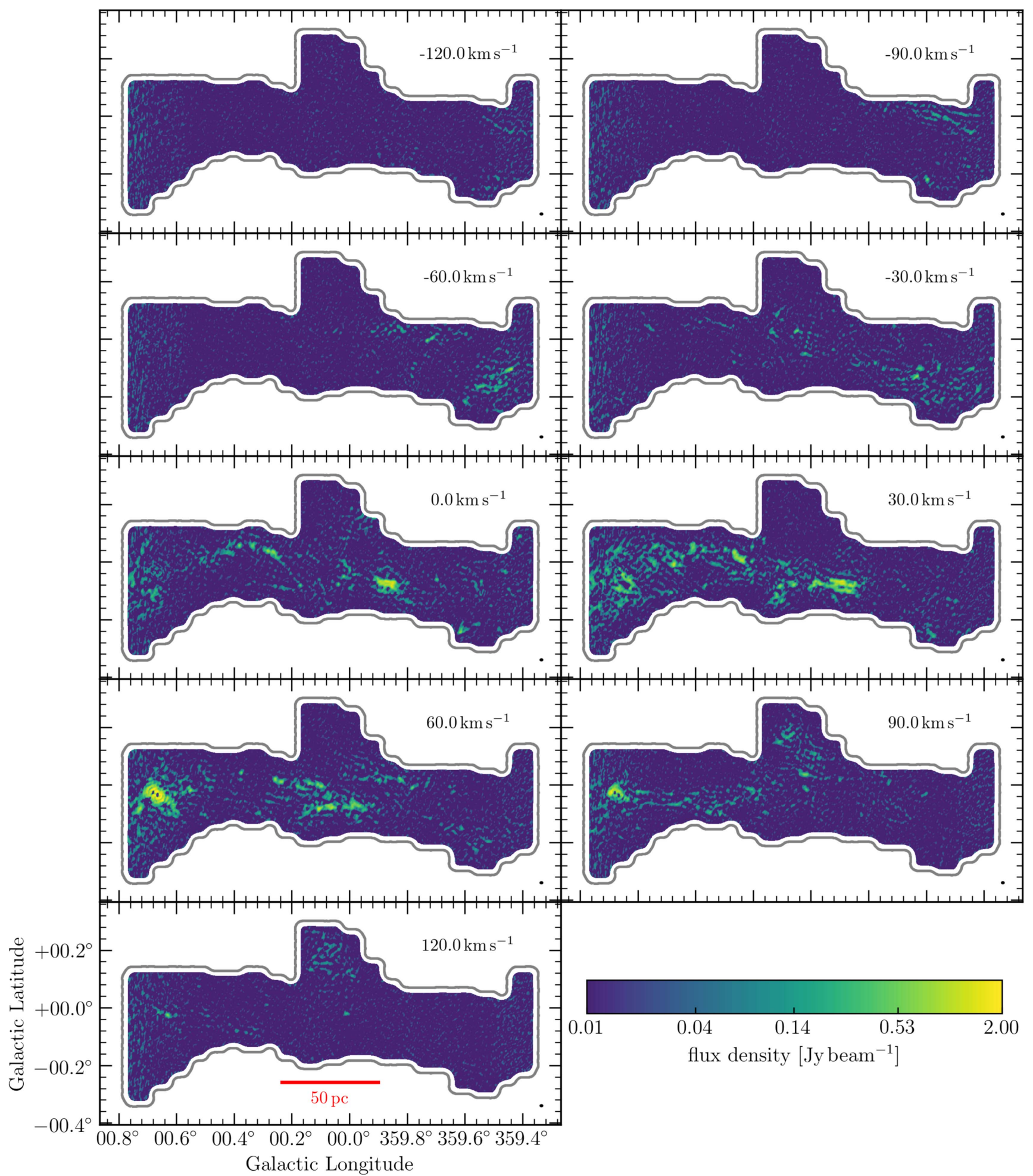

Figure 22. Channel maps of $\mathrm{NH}_{3}(1,1)$. Every fifteenth channel of $2 \mathrm{~km} \mathrm{~s}^{-1}$ (separated by $30 \mathrm{~km} \mathrm{~s}^{-1}$ ) in the range of $-120 \mathrm{~km} \mathrm{~s}^{-1}$ to $+120 \mathrm{~km} \mathrm{~s}^{-1}$ is shown. Velocity and beam area $\left(26^{\prime \prime} .22 \times 17^{\prime \prime} .84,89^{\circ} 3\right)$ are indicated in the top and bottom left corners, respectively. The conversion factor from flux density in Jy beam ${ }^{-1}$ to brightness temperature in $\mathrm{K}$ for this beam size at $23.69 \mathrm{GHz}$ is $T[\mathrm{~K}] / S\left[\mathrm{Jy} \mathrm{beam}^{-1}\right]=4.65$.

measurements, which means that the basic conclusions are not sensitive to the orbital model that is used.

\subsubsection{Sequences at Pericenter Passage}

Qualitatively, increasing temperatures during pericenter passage can occur by converting the gravitational energy released by cloud compression or collapse into heat through a turbulence cascade if cooling is not efficient enough to keep the cloud at a constant temperature (e.g., Ginsburg et al. 2016). The effect on the line width of such a process is not immediately clear and likely needs detailed simulations. In general, turbulence should increase the observed line width, which would result in a rising sequence of line width following the 


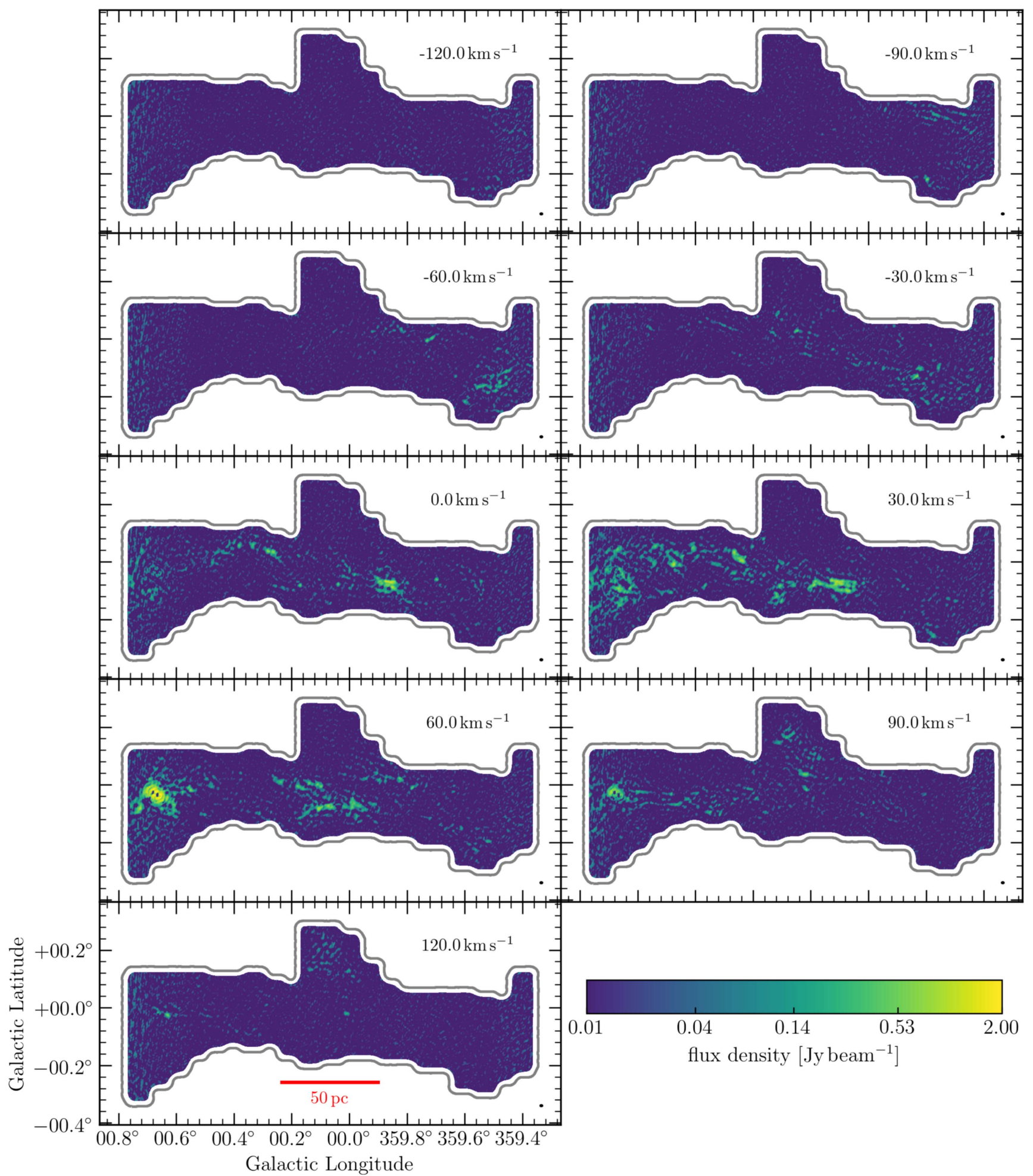

Figure 23. Channel maps of $\mathrm{NH}_{3}(2,2)$. Every fifteenth channel of $2 \mathrm{~km} \mathrm{~s}^{-1}$ (separated by $30 \mathrm{~km} \mathrm{~s}^{-1}$ ) in the range of $-120 \mathrm{~km} \mathrm{~s}^{-1}$ to $+120 \mathrm{~km} \mathrm{~s}$ is shown. Velocity and beam $\left(26^{\prime \prime} 19 \times 17^{\prime \prime} 82,89^{\circ} .3\right)$ are indicated in the top and bottom left corners, respectively. The conversion factor from flux density in Jy beam ${ }^{-1}$ to brightness temperature in $\mathrm{K}$ for this beam size at $23.72 \mathrm{GHz}$ is $T[\mathrm{~K}] / S\left[\mathrm{Jy}\right.$ beam $\left.{ }^{-1}\right]=4.65$.

rise in temperature. However, as the energy makes its way through the cascade to smaller scales $R$, the dissipation rate $\epsilon \propto \sigma / R$ also increases, allowing for little or even no change in the effective line width $\sigma$. If the turbulent line width does increase, it will still be difficult to detect because of variations among clouds (Section 5.2.3).
Radiative cooling generally is an efficient process (e.g., Juvela et al. 2001), and it is not obvious why it could be so inefficient for temperatures to rise by $>50 \mathrm{~K} \mathrm{Myr}^{-1}$. As shown by Figure 14, a significant fraction of clouds in the CMZ are optically thick for line emission at $\sim 25 \mathrm{GHz}$, which can trap cooling photons inside clouds and diminish cooling 


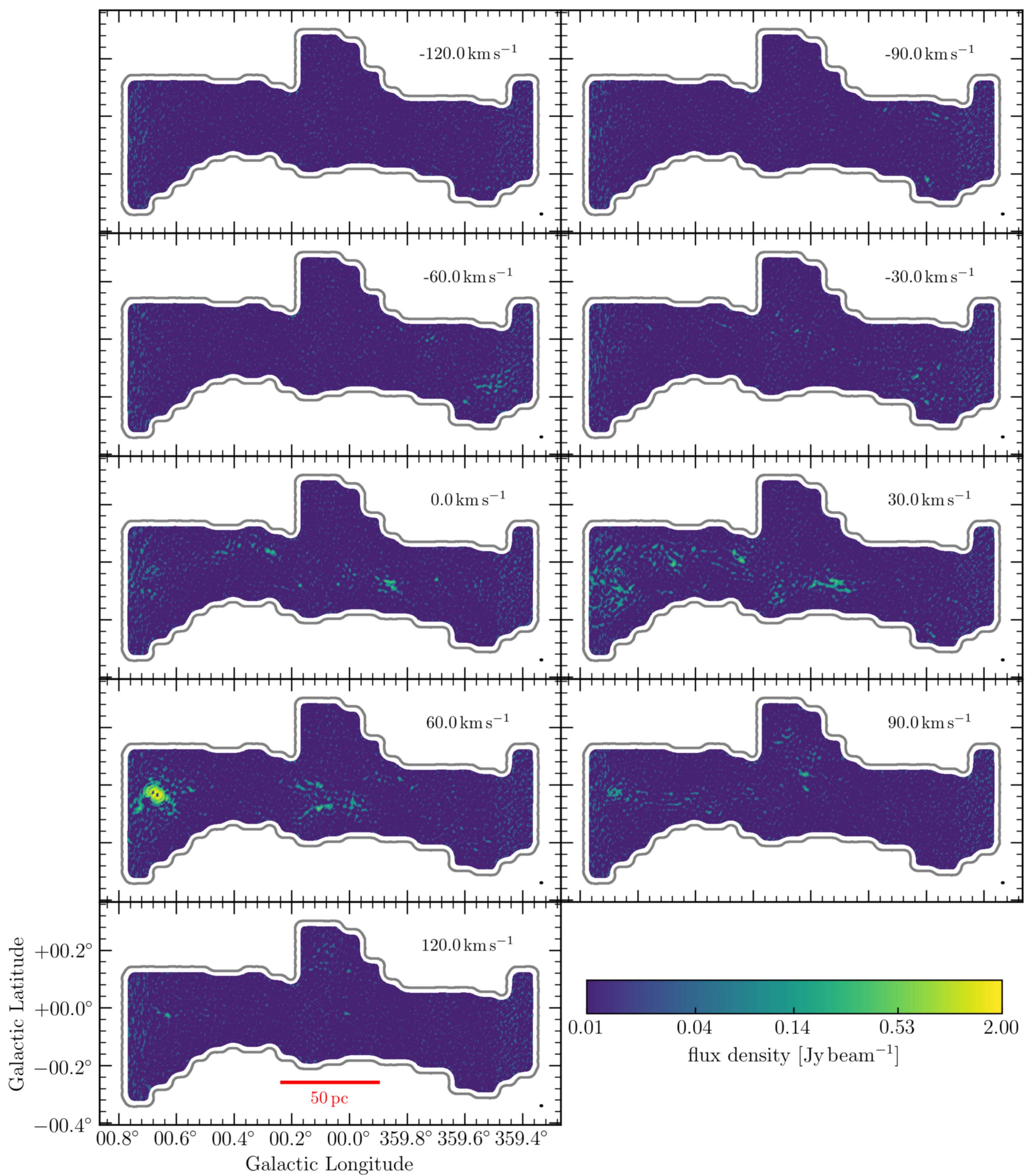

Figure 24. Channel maps of $\mathrm{NH}_{3}(4,4)$. Every fifteenth channel of $2 \mathrm{~km} \mathrm{~s}^{-1}$ (separated by $30 \mathrm{~km} \mathrm{~s}^{-1}$ ) in the range of $-120 \mathrm{~km} \mathrm{~s}^{-1}$ to $+120 \mathrm{~km} \mathrm{~s}$ is shown. Velocity and beam $\left(25^{\prime \prime} 74 \times 17^{\prime \prime} 51,89^{\circ} .3\right)$ are indicated in the top and bottom left corners, respectively. The conversion factor from flux density in Jy beam ${ }^{-1}$ to brightness temperature in $\mathrm{K}$ for this beam size at $24.14 \mathrm{GHz}$ is $T[\mathrm{~K}] / S\left[\mathrm{Jy}\right.$ beam $\left.^{-1}\right]=4.65$.

efficiency. Ammonia is not a dominant cooling line, but other lines that are more important for cooling $\left(\mathrm{CO}, \mathrm{C}^{+}\right.$, and, at least in the Brick, neutral oxygen; Clark et al. 2013) can be expected to be even more optically thick than $\mathrm{NH}_{3}$, and the same argument applies. If true, the hint toward decreasing column density might also be an effect of opacity because in optically thick media, the observed emission originates from an optical depth of $\tau \sim 1$, which is the envelope rather than the core of the cloud. The outer layers of a cloud moving through the pericenter along the stream orbit are sheared and can even become unbound, while the inner cloud is pushed into self-gravity and collapses, as shown observationally by 


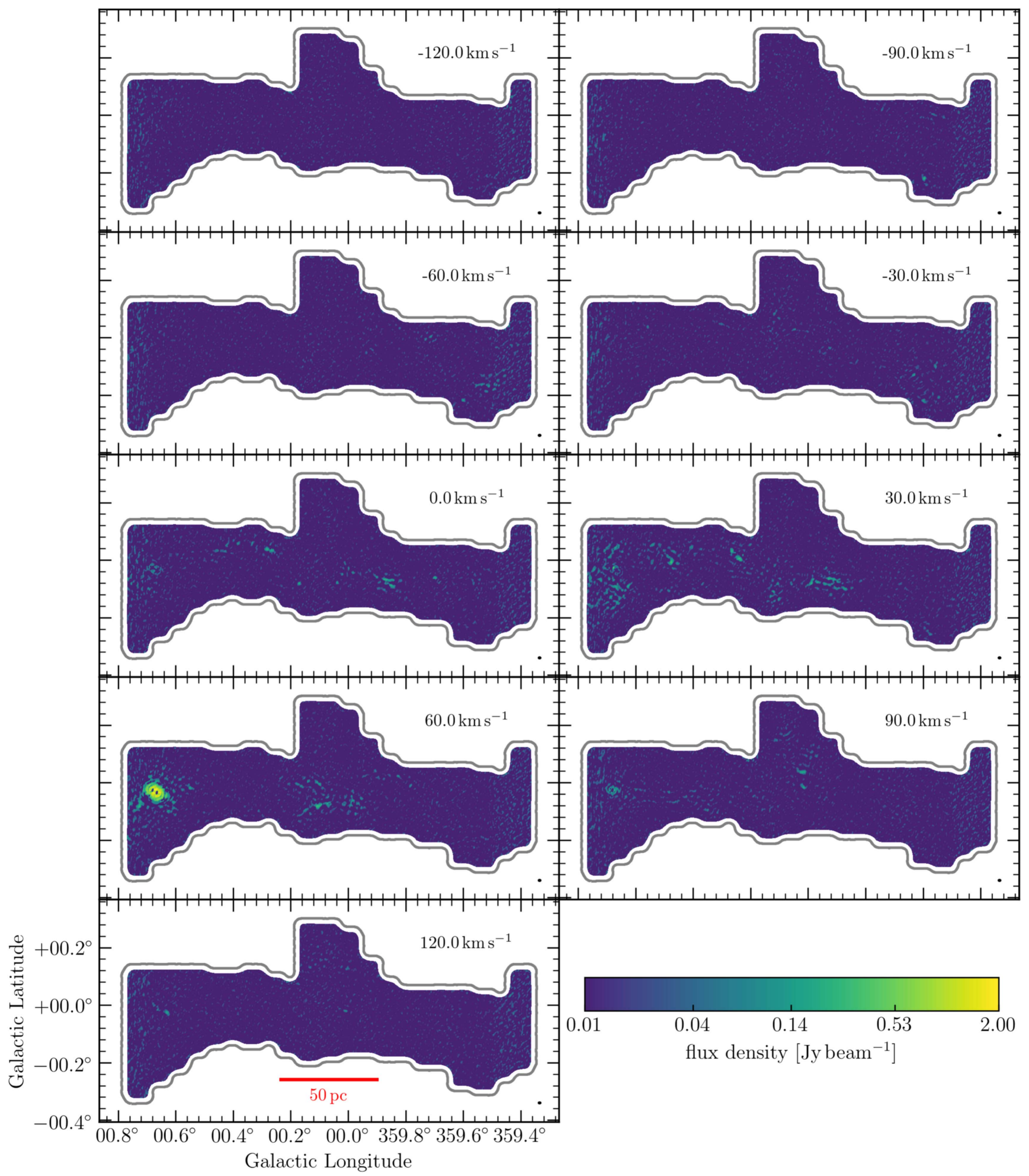

Figure 25. Channel maps of $\mathrm{NH}_{3}(5,5)$. Every fifteenth channel of $2 \mathrm{~km} \mathrm{~s}^{-1}$ (separated by $30 \mathrm{~km} \mathrm{~s}^{-1}$ ) in the range of $-120 \mathrm{~km} \mathrm{~s}^{-1}$ to $+120 \mathrm{~km} \mathrm{~s}^{-1}$ is shown. Velocity and beam $\left(25^{\prime \prime} \cdot 32 \times 17\right.$ !' $\left.23,89^{\circ} .3\right)$ are indicated in the top and bottom left corners, respectively. The conversion factor from flux density in Jy beam ${ }^{-1}$ to brightness temperature in $\mathrm{K}$ for this beam size at $24.53 \mathrm{GHz}$ is $T[\mathrm{~K}] / S\left[\mathrm{Jy}\right.$ beam $\left.^{-1}\right]=4.65$.

Rathborne et al. (2014a), Federrath et al. (2016), and modeled by Kruijssen (2017) and Kruijssen et al. (2017, in preparation). Hence, a trend of decreasing column density can be observed.

The observed mean heating rates at pericenter passage of $\sim 52$ and $\sim 86.3 \mathrm{~K} \mathrm{Myr}^{-1}$ are high, but cannot be easily compared to theoretic predictions because the complex interplay of dynamic and hydrodynamic processes requires simulations that include radiative transfer. Without these simulations, it is also not known at which time before pericenter passage tidal effects start to become non-negligible and affect temperature. The same applies to the time after pericenter passage, when compression and shear 


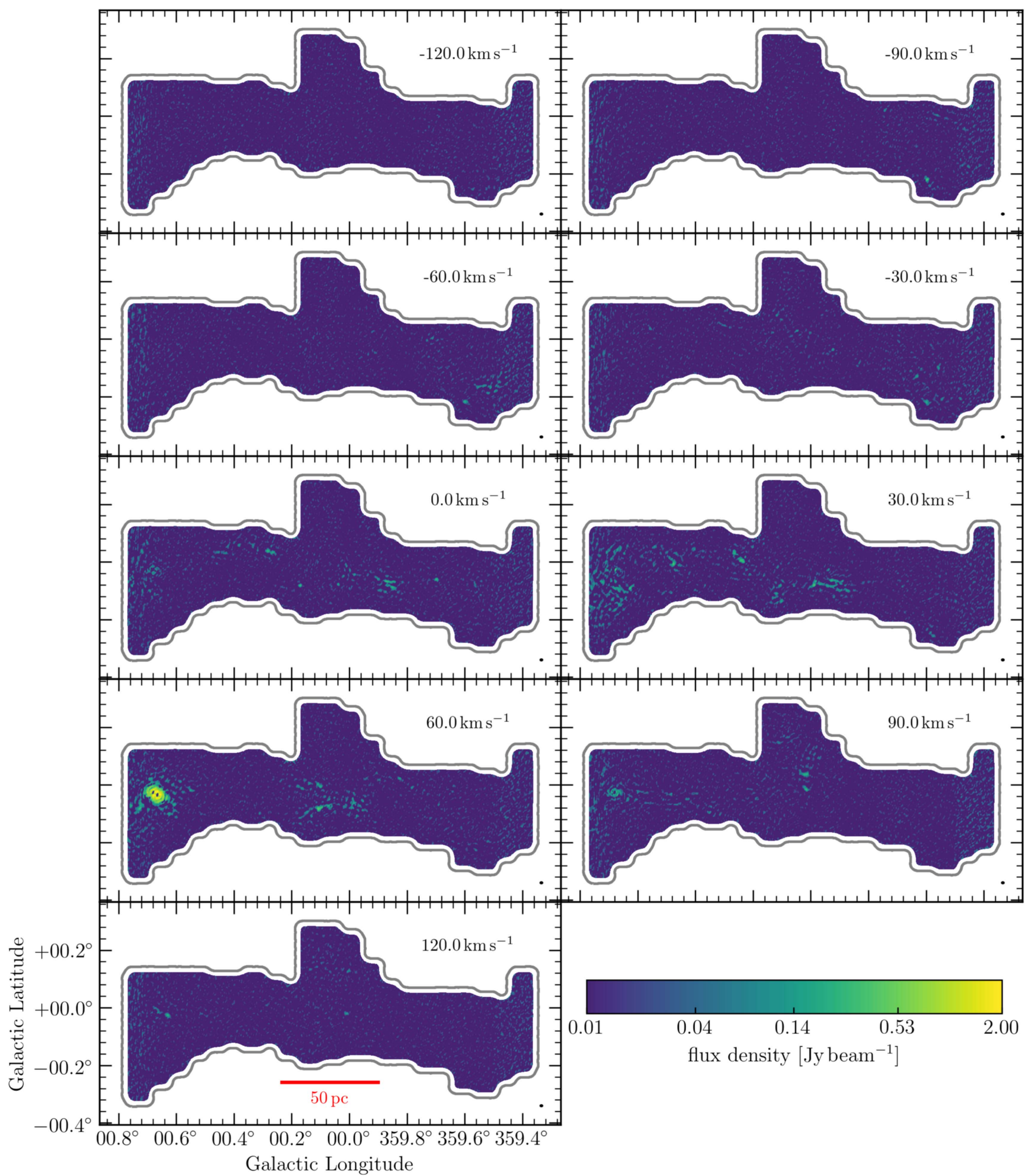

Figure 26. Channel maps of $\mathrm{NH}_{3}(6,6)$. Every fifteenth channel of $2 \mathrm{~km} \mathrm{~s}^{-1}$ (separated by $30 \mathrm{~km} \mathrm{~s}^{-1}$ ) in the range of $-120 \mathrm{~km} \mathrm{~s}^{-1}$ to $+120 \mathrm{~km} \mathrm{~s}$ is shown. Velocity and beam $\left(24 !^{\prime \prime} 80 \times 16^{\prime \prime} 87,89^{\circ} 3\right)$ are indicated in the top and bottom left corners, respectively. The conversion factor from flux density in Jy beam ${ }^{-1}$ to brightness temperature in $\mathrm{K}$ for this beam size at $25.06 \mathrm{GHz}$ is $T[\mathrm{~K}] / S\left[\mathrm{Jy}\right.$ beam $\left.^{-1}\right]=4.65$.

are reduced, which could in principle also lead to cooling, but this is not observed. Detailed simulations can be expected in the near future as the general feasibility was already demonstrated (Clark et al. 2013; Kruijssen 2017). Without simulations, we can estimate the time range of strong tidal influence on the clouds from Figure 20. The indication of increasing temperature in
Figure 20 suggests that a temperature change is detectable at least $0.25 \mathrm{Myr}$ before reaching pericenter. A change in temperature might occur earlier than $0.25 \mathrm{Myr}$ before pericenter passage, but unfortunately, our data do not cover that time range, therefore we cannot check if temperatures are flat before heating sets in. At $0 \mathrm{Myr}$ and $2 \mathrm{Myr}$, the deepest point in the potential is 

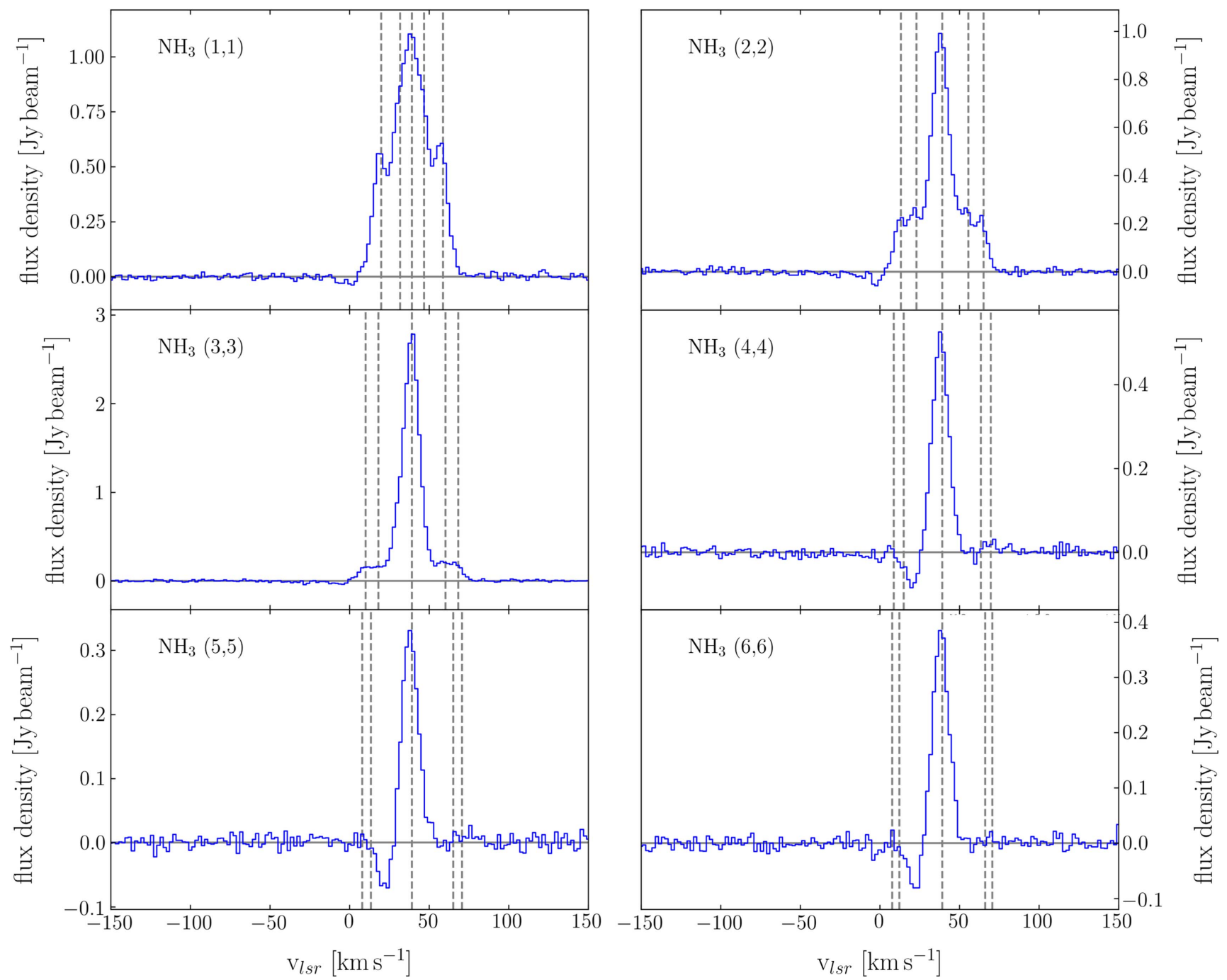

Figure 27. Example spectra with independent scaling of the $y$-axis toward the Brick extracted from the $\mathrm{NH}_{3}(1,1)$ to $(6,6)$ data cubes. The rms noise is $0.062 \mathrm{~K}$, $0.061 \mathrm{~K}, 0.061 \mathrm{~K}, 0.060 \mathrm{~K}, 0.056 \mathrm{~K}$, and $0.061 \mathrm{~K}$, respectively. The positions of the hyperfine satellites according to Table 3 are indicated by dashed vertical lines. Note the complex structure of broad line widths blending hyperfine components and an absorption component at $\sim 20 \mathrm{~km}^{-1}$ in the higher lines.

reached at $R_{\text {peri }} \sim 59 \mathrm{pc}$, while $0.25 \mathrm{Myr}$ corresponds to $R \sim 1.22 R_{\text {peri }}=72 \mathrm{pc}$. Apocenter is reached at $R \sim 120 \mathrm{pc}$, so tidal effects becoming relevant at $\sim 75-80 \mathrm{pc}$ from the center of the potential is plausible. In the spherically symmetric enclosed mass distribution in the GC (Launhardt et al. 2002), the gravitational potential at $\lesssim 0.25 \mathrm{Myr}$ before reaching pericenter ${ }^{29}$ is symmetric to the respective range after pericenter, i.e., tidal effects are expected to play a role until at least $0.25 \mathrm{Myr}$ after pericenter passage. After pericenter passage, some processes reverse, i.e., tidal pressure is released, which can allow cooling, but cloud collapse is ongoing and early star formation may set in. This complex interplay of physical processes makes it very difficult to define an end point of the strong tidal influence from the observational data. Generally, observations show that the temperatures are consistent with turbulent energy dissipation (Ginsburg et al. 2016), as would be expected from triggered cloud collapse.

$\overline{29}$ Or $\sim 50 \mathrm{pc}$ before reaching pericenter at $\sim 200 \mathrm{~km} \mathrm{~s}^{-1}$ orbital velocity, which is appropriate near pericenter; see Kruijssen et al. (2015).

\subsubsection{Sequences after Pericenter Passage}

This observed sequence in the dust ridge starts about one free-fall time $\tau_{\mathrm{ff}}=0.34 \mathrm{Myr}$ after pericenter passage, whereas the other sequences start before. This means that if cloud collapse was triggered, a cloud core collapses, whereas previously sheared but still bound gas is reaccreted. As the collapse of outer cloud layers proceeds, the release of gravitational energy declines, which implies flattening temperature gradients. However, no obvious decrease in heating rate is found in the dust ridge, meaning that cloud collapse is still ongoing at a similar intensity as at pericenter passage, or alternatively, that a second heating source starts to contribute. In the framework of triggered SF, star formation could be a second source of heating. As an estimate, star formation can set in as early as one free-fall time after the cloud itself started to collapse, which is $0.34 \mathrm{Myr}$ for a typical CMZ cloud, and this coincides with the time since the Brick passed pericenter (K15). Accordingly, early signs of embedded star formation have been found in one region of the Brick (Longmore et al. 2012; Johnston et al. 2014; Rathborne et al. 2014a), i.e., heating by star formation is just about to set in. 

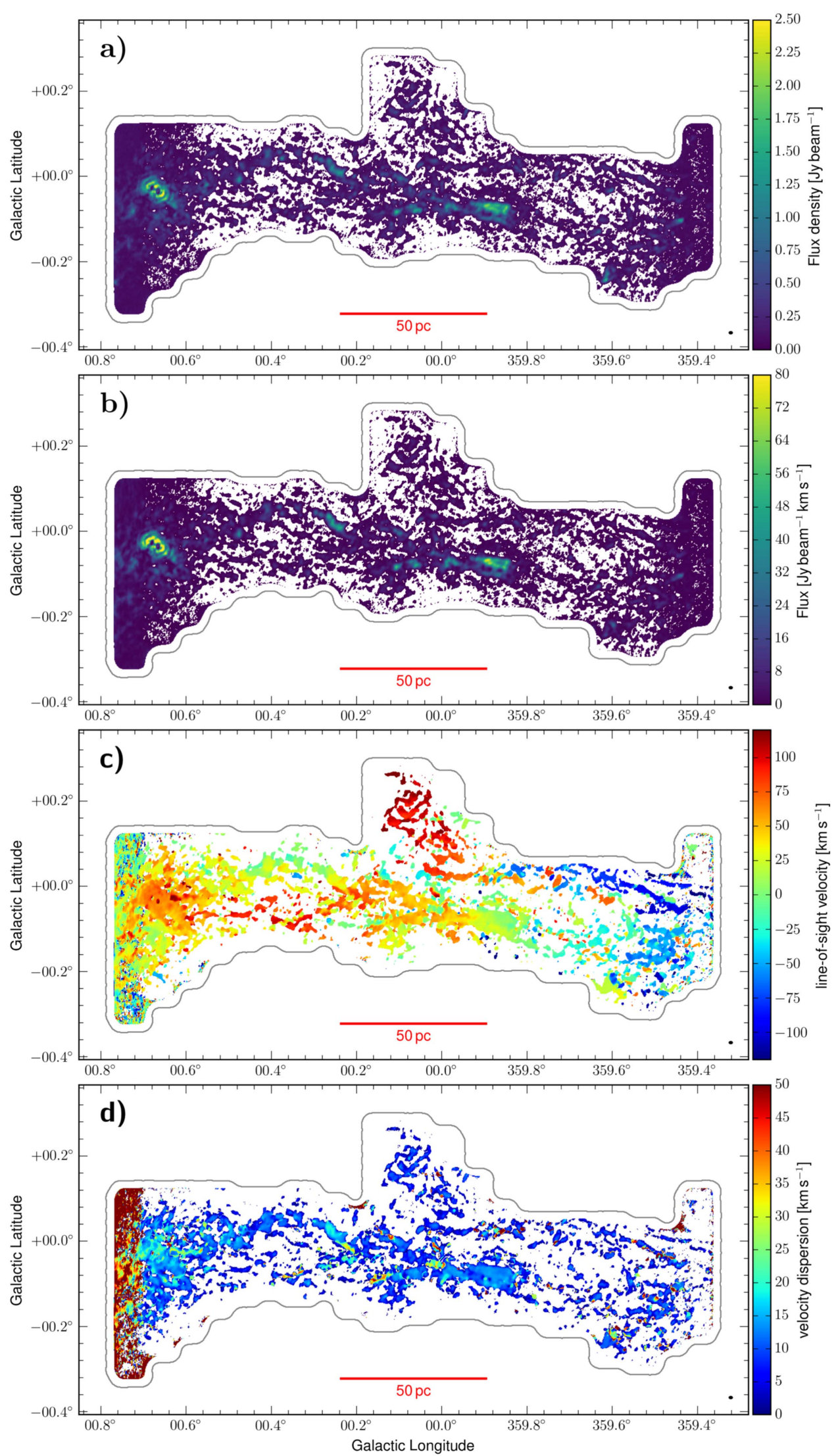

Figure 28. $\mathrm{NH}_{3}(1,1)$ moment maps: (a) peak intensity, (b) integrated intensity (moment 0 ), (c) intensity-weighted mean velocity (moment 1), and (d) intensityweighted velocity dispersion (moment 2). The intensity maps (a) and (b) are masked at $3 \sigma$, velocity maps (c) and (d) are masked at $5 \sigma$ with an rms noise of $\sigma=13.4 \mathrm{mJy}$. The beam of $26^{\prime \prime}, 2 \times 17$ !! $8(1.06 \mathrm{pc} \times 0.72 \mathrm{pc})$ is indicated in the lower right corner of each panel. 

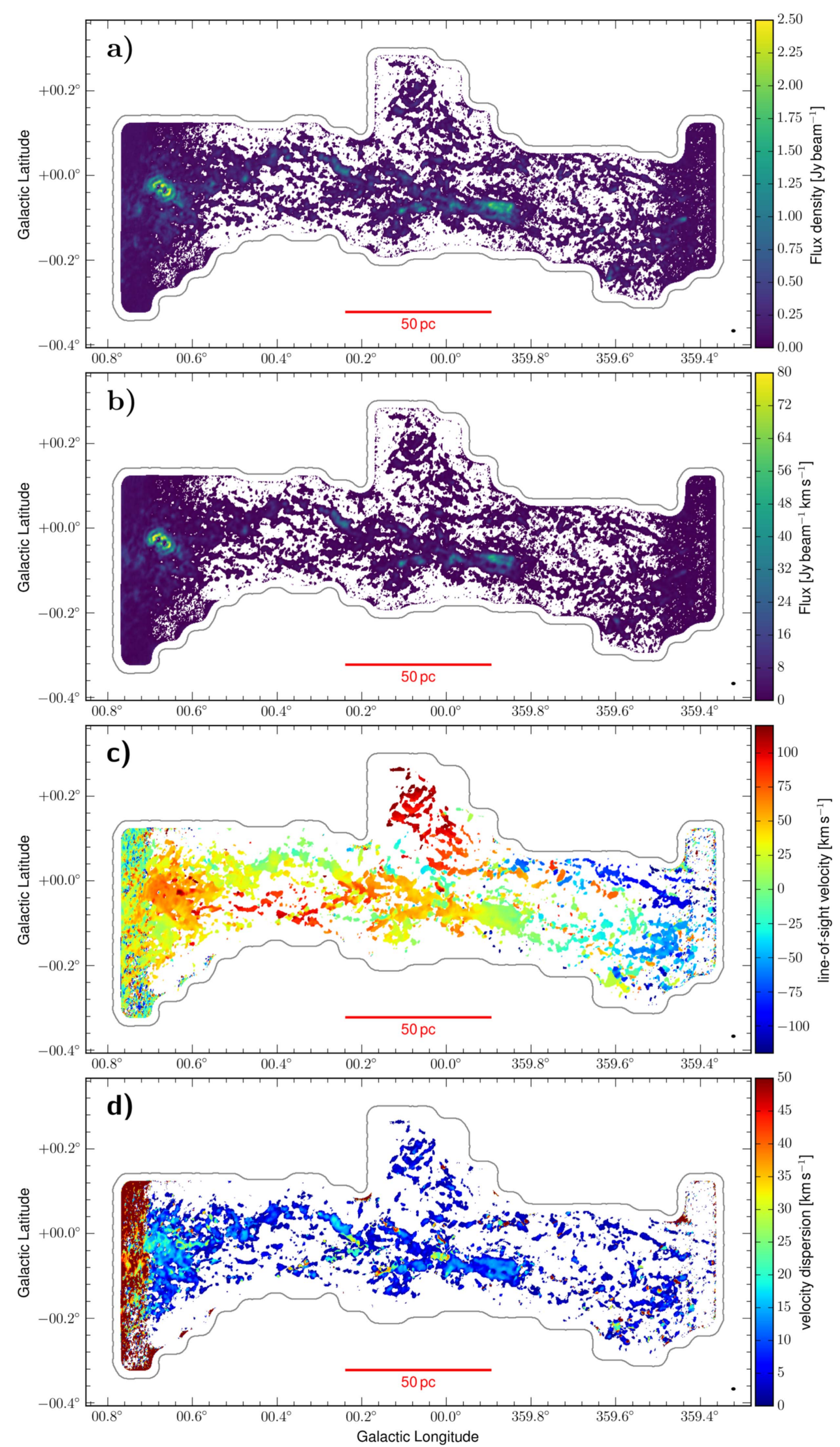

Figure 29. $\mathrm{NH}_{3}(2,2)$ moment maps: (a) peak intensity, (b) integrated intensity (moment 0 ), (c) intensity-weighted mean velocity (moment 1 ), and (d) intensityweighted velocity dispersion (moment 2). The intensity maps (a) and (b) are masked at $3 \sigma$, velocity maps (c) and (d) are masked at $5 \sigma$ with an rms noise of $\sigma=13.1 \mathrm{mJy}$. The beam of $26.2 \times 17$ !' $8(1.05 \mathrm{pc} \times 0.72 \mathrm{pc})$ is indicated in the lower right corner of each panel. 



Figure 30. $\mathrm{NH}_{3}(4,4)$ moment maps: (a) peak intensity, (b) integrated intensity (moment 0 ), (c) intensity-weighted mean velocity (moment 1 ), and (d) intensity-weighted velocity dispersion (moment 2). The intensity maps (a) and (b) are masked at $3 \sigma$, velocity maps (c) and (d) are masked at $5 \sigma$ with an rms noise of $\sigma=12.9$ mJy. The beam of $25^{\prime \prime} .7 \times 17 ! \prime 5(1.04 \mathrm{pc} \times 0.70 \mathrm{pc})$ is indicated in the lower right corner of each panel. 

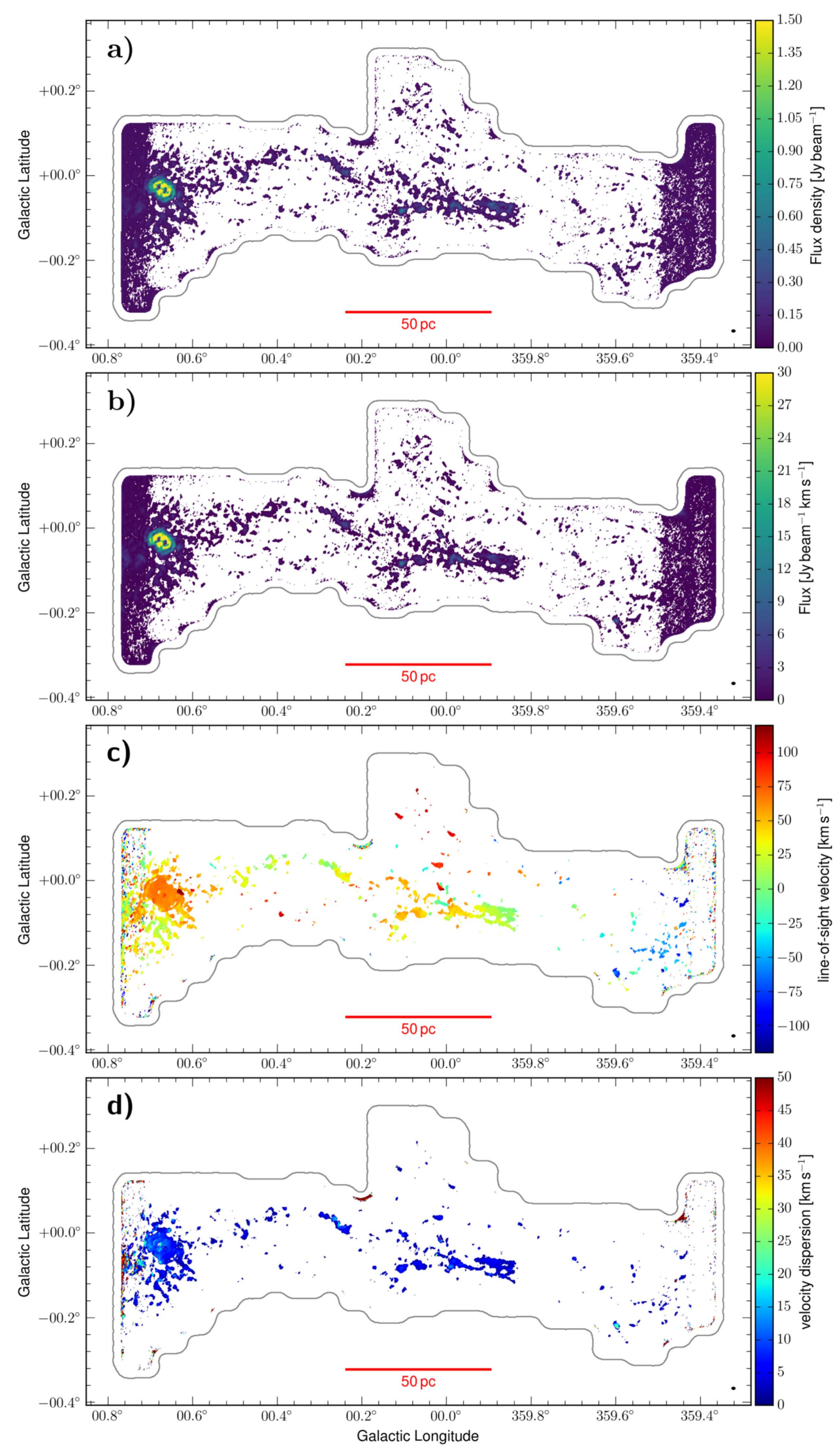

Figure 31. $\mathrm{NH}_{3}(5,5)$ moment maps: (a) peak intensity, (b) integrated intensity (moment 0 ), (c) intensity-weighted mean velocity (moment 1$)$, and (d) intensity-weighted velocity dispersion (moment 2). The intensity maps (a) and (b) are masked at $3 \sigma$, velocity maps (c) and (d) are masked at $5 \sigma$ with an rms noise of $\sigma=12.0$ mJy. The beam of $25^{\prime \prime} .3 \times 17$ !' $2(1.02 \mathrm{pc} \times 0.69 \mathrm{pc})$ is indicated in the lower right corner of each panel. 

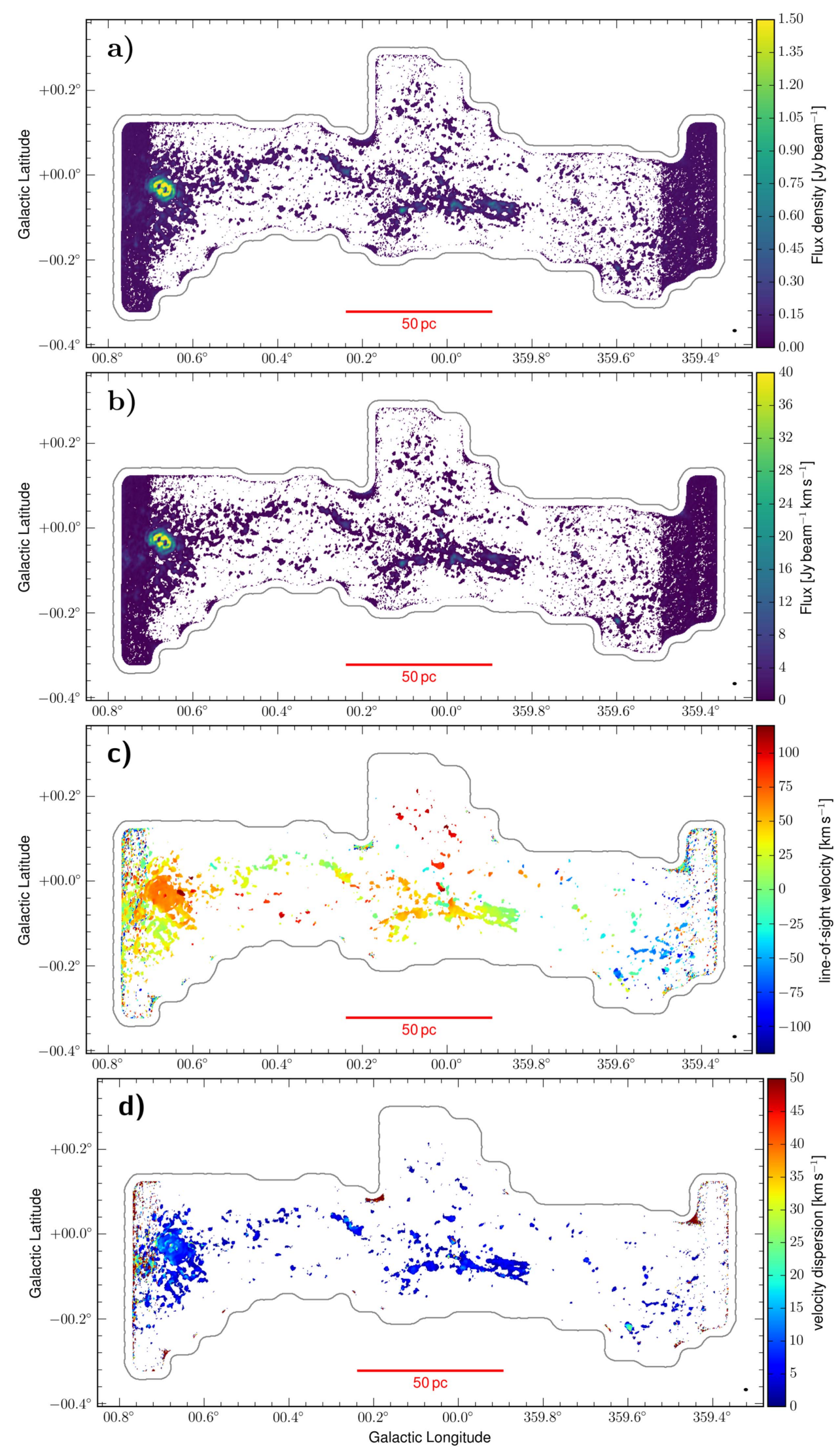

Figure 32. $\mathrm{NH}_{3}(6,6)$ moment maps: (a) peak intensity, (b) integrated intensity (moment 0 ), (c) intensity-weighted mean velocity (moment 1), and (d) intensity-weighted velocity dispersion (moment 2). The intensity maps (a) and (b) are masked at $3 \sigma$, velocity maps (c) and (d) are masked at $5 \sigma$ with an rms noise of $\sigma=13.2 \mathrm{mJy}$. The beam of $24 ! \prime 8 \times 16^{\prime \prime} 9(1.00 \mathrm{pc} \times 0.68 \mathrm{pc})$ is indicated in the lower right corner of each panel. 
These effects increase the temperature if the gas cannot cool efficiently enough, which apparently is the case as gas temperatures are known to be significantly offset from dust temperatures (Figure 17 and e.g., Güsten et al. 1981; Molinari et al. 2011; Ginsburg et al. 2016). Therefore, the increase in temperature cannot simply be attributed to a single of these processes, but to a combination at varying relative strengths, which is not known at the moment for GC conditions and cannot be inferred from our observations. In order to disentangle the contribution of star formation from the kinematic and gravitational effects, a complex hydrodynamic gas simulation including star formation feedback is needed.

Collapsing clouds are expected to show a signature of increasing column density that is not observed, which might be a consequence of shearing outer cloud layers during pericenter passage. It might also be an observational effect of too low resolution to trace the collapse. Collapse occurs quickest on the highest density, lowest virial ratio scales. It is known in the literature that the column density profiles of the Brick and bricklet clouds are centrally concentrated and that the volume density of the Brick increases toward the center (Walker et al. 2015; Rathborne et al. 2016). The collapse will therefore occur quickest at the center, on scales smaller than the SWAG beam, which will wash out potential small-scale effects. In higher resolution observations, Walker et al. (2015) found evidence for increasing gas and (proto-) stellar density along the stream orbit from the Brick to Sgr B2.

As discussed in Section 5.2.1, the sequence of increasing temperature around $\mathrm{Sgr} \mathrm{C}$ is likely not connected to the sequence at near-side pericenter because of the sharp discontinuity and differing heating rate. The observation of $24 \mu \mathrm{m}$ emission in this region and the higher temperature compared to the pericenter sequence suggest that these clouds already underwent tidal triggering of collapse at far-side pericenter (see also Kruijssen et al. (2015) regarding the position of Sgr C). This places $\mathrm{Sgr} \mathrm{C}$ as a successor to the dust ridge sequence in terms of evolution, but not in time. Finding no column density trend in Sgr C matches the picture, as this cloud is supposedly collapsed already and is actively forming stars (Kendrew et al. 2013; Lu et al. 2017), which powers the rise in temperature.

\subsubsection{Connection of Sequences at and after Pericenter Passage}

According to the K15 model, the empirically found sequences are directly associated in the sense that the phasse dominated by star formation (dust ridge and Sgr C) follows the phase dominated by tidal compression (near and far pericenter passage). The orbital phase, however, cannot be the only parameter controlling the physical state of gas along the stream, as discussed in Section 5.2.1. It seems likely that the clouds at and after pericenter passage are similar but not identical in properties, i.e., all clouds may follow the same evolution, but the gas in the dust ridge was initially cooler or could cool more efficiently when cloud collapse was triggered. The offset in temperature that is required to match the sequences is small $(\sim 15-20 \mathrm{~K})$ compared to the observed heating rates $\left(\sim 52 \mathrm{~K} \mathrm{Myr}^{-1}\right)$. As we do not know the initial cloud properties, this remains speculation, and we can only state that the observed sequences can be the result of tidally triggered collapse assuming a scatter of initial cloud temperature of $\sim 20 \mathrm{~K}$. On this note, it should be kept in mind that individual cloud properties as well as collapse triggering are statistically distributed and are not identical for subsequent occurrences.
Table 3

Ammonia Hyperfine Components as Calculated from Tabulated Data in Townes \& Schawlow (1975) and splatalogue.net

\begin{tabular}{|c|c|c|c|c|c|c|}
\hline \multirow{2}{*}{ Line } & \multicolumn{5}{|c|}{ Component } & \\
\hline & -2 & -1 & 0 & 1 & 2 & \\
\hline \multirow{2}{*}{$\begin{array}{l}\mathrm{NH}_{3} \\
\quad(1,1)\end{array}$} & -19.48 & -7.46 & 0 & 7.59 & 19.61 & {$\left[\mathrm{~km} \mathrm{~s}^{-1}\right]$} \\
\hline & 0.111 & 0.139 & 0.500 & 0.139 & 0.111 & \\
\hline \multirow{2}{*}{$\begin{array}{l}\mathrm{NH}_{3} \\
\quad(2,2)\end{array}$} & -25.91 & -16.30 & 0 & 16.43 & 26.03 & {$\left[\mathrm{~km} \mathrm{~s}^{-1}\right]$} \\
\hline & 0.052 & 0.054 & 0.789 & 0.054 & 0.052 & \\
\hline \multirow{2}{*}{$\begin{array}{l}\mathrm{NH}_{3} \\
\quad(3,3)\end{array}$} & -29.14 & -21.10 & 0 & 21.10 & 29.14 & {$\left[\mathrm{~km} \mathrm{~s}^{-1}\right]$} \\
\hline & 0.027 & 0.029 & 0.888 & 0.029 & 0.027 & \\
\hline \multirow{2}{*}{$\begin{array}{l}\mathrm{NH}_{3} \\
\quad(4,4)\end{array}$} & -30.43 & -24.22 & 0 & 24.22 & 30.43 & {$\left[\mathrm{~km} \mathrm{~s}^{-1}\right]$} \\
\hline & 0.016 & 0.016 & 0.935 & 0.016 & 0.016 & \\
\hline \multirow{2}{*}{$\begin{array}{l}\mathrm{NH}_{3} \\
\quad(5,5)\end{array}$} & -31.41 & -25.91 & 0 & 25.91 & 31.41 & {$\left[\mathrm{~km} \mathrm{~s}^{-1}\right]$} \\
\hline & 0.011 & 0.011 & 0.956 & 0.011 & 0.011 & \\
\hline \multirow{2}{*}{$\begin{array}{l}\mathrm{NH}_{3} \\
\quad(6,6)\end{array}$} & -31.47 & -26.92 & 0 & 26.92 & 31.47 & {$\left[\mathrm{~km} \mathrm{~s}^{-1}\right]$} \\
\hline & 0.008 & 0.008 & 0.969 & 0.008 & 0.008 & \\
\hline
\end{tabular}

Note. For each ammonia species, the top row lists spectral offset in $\mathrm{km} \mathrm{s}^{-1}$, the bottom row shows the fractional line strength.

Estimates for the variation of physical parameters from cloud to cloud can be drawn from the analysis of velocity corrugations in the $\left[l^{-}, b^{-}\right]$segment of the stream, which is expected to be a precursor to the dust ridge (Henshaw et al. 2016a). For this analysis, Kruijssen (2017) report standard deviations of initial cloud parameters of $0.19 \mathrm{dex}$ (mass), $0.09 \mathrm{dex}$ (radius), 0.16 dex (density), and 0.08 dex (free-fall timescale) among the potential precursors to the dust ridge only. The standard deviations would naturally be larger when the progenitors to the other temperature sequences identified in this paper were also included. If the variations of initial cloud temperature are at a similar level as these properties, the expected temperature scatter is $\gtrsim 20 \mathrm{~K}$, larger than the observed offsets of $15-20 \mathrm{~K}$. These numbers show that the observed temperature sequences at and after pericenter passage may be causally related as the temperature deviation is within the expected scatter.

\subsubsection{Orbital Phase and Variation of the Trigger Point}

The periodic occurrence of pericenter passages every $\sim 2.0$ Myr suggests that temperatures can also be plotted against orbital phase, which is shown in Figure 21 (top panel). Although four temperature sequences could be defined as a function of absolute time, no global sequence is found. Only the time range 0.5 to $0.9 \mathrm{Myr}$ shows a consistent trend of rising temperatures as the data in this region originate almost exclusively from the dust ridge. The other sequences are lost because differing temperature levels are superposed, which also smears the discontinuity at $t_{\text {peri }}=1.75 \mathrm{Myr}$ that occurs at a phase of $t_{\text {last }}=-0.25 \mathrm{Myr}$. However, fitting a linear relation in the time range -0.25 to $0.90 \mathrm{Myr}$ (sum of the four ranges identified in Section 5.2) still results in a positive heating rate of $14.6 \mathrm{~K} \mathrm{Myr}^{-1}$. Almost identical results are obtained for $T_{45}$ and $T_{36}$ with relative errors of $\sim 100 \%$, as listed in Table 2 . These results are in line with Kauffmann et al. (2017b), who find a trend in density gradients of six CMZ clouds, but no clear trend in star formation activity per unit dense gas as a function of orbital phase. 


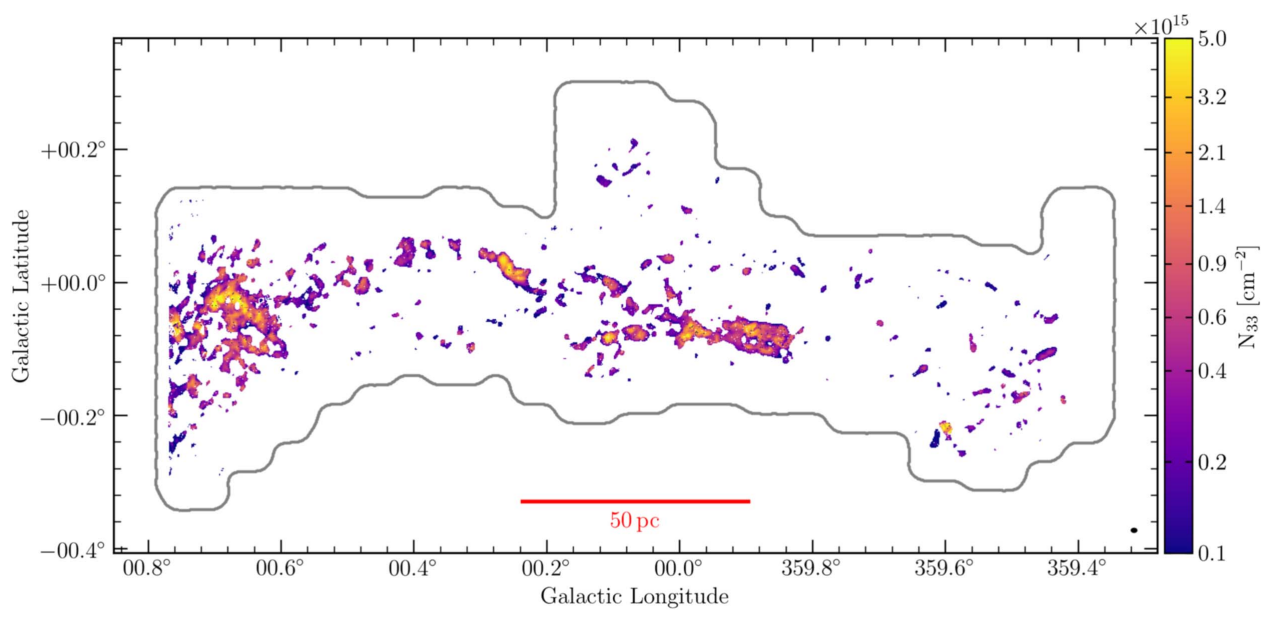

Figure 33. $\mathrm{NH}_{3}(3,3)$ column density $N_{l}$ under consideration of non-zero opacity as derived by Equation (18) from fitting the hyperfine structure.

The signature of rising temperature could be expected to become stronger by transforming into orbital phase if the four sequences are samples of a single consistent evolution. However, it is highly unlikely that all clouds are triggered to collapse exactly at pericenter, this is expected to occur rather within some time range around pericenter. By allowing the zero-point $t_{\text {start }}$ of each sequence to vary by up to $\pm 0.3 \mathrm{Myr}$, the sequential behavior can be recovered (Figure 21 bottom panel). The heating rate is maximized at a value of $\sim 48 \mathrm{~K} \mathrm{Myr}^{-1}$ for zero-points of $t_{\text {start }}=-0.3 \mathrm{Myr}$ (dust ridge), $t_{\text {start }}=+0.2 \mathrm{Myr}$ (pericenter near) and $t_{\text {start }}=+0.2 \mathrm{Myr}$ (pericenter far). At $0.3 \mathrm{Myr}$ before or after pericenter, the radial distance from the bottom of the GC potential is $R=77 \mathrm{pc}(R=60 \mathrm{pc}$ at pericenter), and thus the clouds are still deep in the potential where tidal forces can be expected to be sufficiently strong to trigger cloud collapse. The fact that heating rates similar to the individual values can be recovered by only adjusting the exact trigger point without taking potential variations of initial cloud conditions or variations of the gravitational potential into account suggests that $t_{\text {start }}$ is one of the major sources of scatter of a downstream $\mathrm{SF}$ sequence. The stacked and $t_{\text {start }}$-adjusted mean heating rates are lower than the per-segment heating rates, implying that another factor still erases part of the trend. This is the vertical (temperature) offset, which is due to slightly different initial conditions, as has been discussed in Section 5.3.3. This observation confirms the warning by Kruijssen (2017) that simple stacking of pericenter passages is not advisable because of scatter. However, problems can be diminished if it is possible to estimate the scatter in $t_{\text {start }}$. Additional scatter can result from the local environment (e.g., radiation field), which does not have to be identical for two clouds of the same evolutionary stage at different positions. For instance, pericenters on the near and far side are identified with each other in terms of evolutionary stage despite the spatial separation of $\sim 120 \mathrm{pc}$. Hence, influences of the environment can be very different at a given time since the last pericenter passage.

\subsection{Sequential Evolution in other CMZ Models}

Although this analysis is based on the kinematic stream model of K15, similar results would be obtained assuming a different orbital model. ${ }^{30}$ However, the interpretation of the

\footnotetext{
${ }^{30}$ Of course, this also assumes that a consensus about the $3 \mathrm{D}$ position of CMZ clouds is reached. Results differ greatly when a cloud is placed on the far side of the GC instead of in front.
}

sequences we found in Section 5.3 relies on a varying tidal field strength along the orbit. In the context of a dense gas torus (Molinari et al. 2011) instead of streams, the basic requirement for a tidal triggering of cloud collapse is still present (as argued by Longmore et al. 2013b): gas passes close to the bottom of the GC gravitational potential. It is irrelevant if this is due to a displaced orbit (ring model), elliptical orbits $\left(x_{1} / x_{2}\right.$ orbits in the bar model), or a radially oscillating stream. Differing dynamics (e.g., orbital speed) imply that the time axis of Figure 20 is stretched or squished, but the progression fundamentally stays the same. The major difference in the context of this work is the question whether another trigger point on the far side of the orbit is present, which does not strongly affect this analysis as most of the gas is detected in the dust ridge or near-side pericenter. Thus, a definite distinction between models is not possible, but the hints of a third trigger point at far-side pericenter passage favors the K15 model. As not all the models agree on the clouds' positions along the line of sight, any connection between the sequences we found relies on the adopted model. Some versions of the two spiral arms model place the 20 and $50 \mathrm{~km} \mathrm{~s}^{-1}$ clouds or Sgr C and the dust ridge on different arms (Sofue 1995; Sawada et al. 2004), thus no connection is given, whereas in a recent spiral arm model (Ridley et al. 2017) and the elliptical ring model, the dust ridge immediately follows the 20 and $50 \mathrm{~km} \mathrm{~s}^{-1}$ clouds. The K15 model is different from all other published orbital models of the CMZ in that it predicts two different pericenters on the near side. This is consistent with our observations, but the absence of dense gas on segment 2 before the dust ridge does not allow a definite distinction between models.

\subsection{Relation to Other Recent GC SF Research}

Finding a gas temperature sequence in the CMZ stream segments confirms implications that followed from the star formation triggering model by Longmore et al. (2013b), but does not directly confirm that the clouds themselves evolve as expected. Heating or cooling of molecular clouds does not necessarily have to be associated with star formation or collapse in the theoretically expected way, but can be related to other mechanisms such as cosmic ray heating as well (e.g., Yusef-Zadeh et al. 2007; Ao et al. 2013; Clark et al. 2013). A sequence of rising temperature could be observed as a function of distance to the potential heating sources Sgr A* and Sgr B2. For these two sources and the distribution of measurements, the 
Table 4

Conversion Equations for the Rotational to Kinetic Temperature Conversion as Obtained by Ott et al. (2011) and Partially Published in Ott et al. (2011) and Gorski et al. (2017)

\begin{tabular}{|c|c|c|c|c|c|}
\hline \multicolumn{3}{|c|}{$T_{\text {kin }}-T_{\text {rot }}$ conversion } & \multicolumn{3}{|c|}{ Temperature Range } \\
\hline$T_{\text {kin, } 12}$ & $=$ & $6.05 \cdot \exp \left(0.06088 T_{\text {rot, } 12}\right)$ & & & \\
\hline \multirow[t]{2}{*}{$T_{\text {kin }, 24}$} & $=$ & $1.467 T_{\text {rot, } 24}-6.984$ & $0 \mathrm{~K} \lesssim$ & $T_{\text {kin }}$ & $\lesssim 100 \mathrm{~K}$ \\
\hline & $=$ & $27.085 \cdot \exp \left(0.019 T_{\text {rot }, 24}\right)$ & $100 \mathrm{~K} \lesssim$ & $T_{\text {kin }}$ & $\lesssim 500 \mathrm{~K}$ \\
\hline \multirow[t]{2}{*}{$T_{\text {kin }, 36}$} & $=$ & $T_{\text {rot }, 36}$ & $0 \mathrm{~K} \lesssim$ & $T_{\text {kin }}$ & $\lesssim 50 \mathrm{~K}$ \\
\hline & $=$ & $28.87511 \cdot \exp \left(0.015 T_{\text {rot }, 36}\right)$ & & $T_{\text {kin }}$ & $>50 \mathrm{~K}$ \\
\hline \multirow[t]{2}{*}{$T_{\text {kin }, 45}$} & $=$ & $1.143 T_{\text {rot }, 45}-1.611$ & $0 \mathrm{~K} \lesssim$ & $T_{\text {kin }}$ & $\lesssim 50 \mathrm{~K}$ \\
\hline & $=$ & $21.024 \cdot \exp \left(0.0198 T_{\text {rot }, 45}\right)$ & $50 \mathrm{~K} \lesssim$ & $T_{\text {kin }}$ & $\lesssim 500 \mathrm{~K}$ \\
\hline
\end{tabular}

Table 5

Fit Success Rates for the Six Ammonia Inversion Lines Observed with SWAG

\begin{tabular}{lcccccc}
\hline \hline & $(1,1)$ & $(2,2)$ & $(3,3)$ & $(4,4)$ & $(5,5)$ & $(6,6)$ \\
\hline Selected pixels & 24383 & 24383 & 24383 & 24383 & 24383 & 24383 \\
Fitted pixels & 23790 & 23025 & 23646 & 19269 & 19261 & 19245 \\
Failed to fit & 593 & 1358 & 917 & 5114 & 5122 & 5138 \\
$\%$ fitted & 97.6 & 94.4 & 96.2 & 79.0 & 79.0 & 78.9 \\
Good fits & 23437 & 22822 & 23403 & 19137 & 18795 & 18971 \\
Bad fits & 353 & 203 & 63 & 132 & 466 & 274 \\
$\%$ good & 98.5 & 99.1 & 99.7 & 99.3 & 97.6 & 98.6 \\
\hline
\end{tabular}

Note. 24,383 pixels were selected by a two-step $\mathrm{S} / \mathrm{N}$ criterion to be fitable in at least one of the lines $\mathrm{NH}_{3}(1,1)$ to $\mathrm{NH}_{3}(6,6)$. The actual number of attempted fits per ammonia species ("fitted pixels," second row) is lower because of nonidentical distributions and decreasing $\mathrm{S} / \mathrm{N}$ for higher states. The difference is given in rows 3 and 4 ("failed to fit" and success percentage) and lists the amount of pixels that could not be fitted because they were excluded by masking. Out of the attempted fits, the vast majority of $>98.5 \%$ did converge and yielded physical plausible results ("good fits"); "bad" fits are defined by large uncertainty and physically implausible values (see Section 4.1 for details).

fitted time since pericenter passage can be related to physical distance. The observed temperature sequence in the dust ridge rises toward Sgr B2, which is qualitatively consistent with it being the locally dominating heating source. The other sequences, however, do not match this picture because the molecular clouds on the far side show an inverse trend and gas at negative longitudes requires a second heating source to the east that does not influence the close-by western end of the dust ridge. Similar arguments apply to Sgr A*. Clouds are observed to heat up beyond pericenter (closest distance to $\mathrm{Sgr}^{*}$ ), and the 20 and $50 \mathrm{~km} \mathrm{~s}^{-1}$ clouds are colder than Sgr C, which is $\sim 1.5$ times farther from Sgr $\mathrm{A}^{*}$. However, these arguments also rely on the 3D distribution of gas in the GC, which is still debated (e.g., McGary et al. 2001; Clavel et al. 2013; Kruijssen et al. 2015; Takekawa et al. 2015; Churazov et al. 2017).

It is therefore essential to gain more insight into cloud evolution in the stream and the GC in general in order to link it to observations of star formation tracers (in the dust ridge) and stars (Arches and Quintuplet clusters). Barnes et al. (2017) extend the SF sequence downstream from Sgr B2 by finding evidence of a gradual increase in stellar feedback activity as measured from the size of the hot gas bubbles driven by young massive stars. The Arches and Quintuplet clusters are found to be consistent in kinematics and age to have formed by tidally triggered cloud collapse and thus seem to fit in the GC star formation model (K15). If this can be confirmed by future observations, the time range covered by the model would greatly increase from pre-collapse clouds ( $t_{\text {peri }} \sim-1$ to $-2 \mathrm{Myr}$ ) to evolved stars (Quintuplet, $t_{\text {peri }} \sim 5 \mathrm{Myr}$ ) and span the whole time range of star formation. Immediate insights for cloud evolution could be gained from a study of internal cloud kinematic, size, shape, and density.

In addition to the ISM ingredients gas and dust, the key to a better understanding of (proto-) star formation in extreme environments such as the CMZ are the stars themselves. Evolution in gas and possibly also in dust is accompanied by increasing signs of star formation along the dust ridge that we suspect to partially power the rise in temperature. Starting from an increased base level after initial heating by tidal effects, the Brick is still cold and shows little star formation (Longmore et al. 2012; Kauffmann et al. 2013; Rodríguez \& Zapata 2013), while cloud c contains $\mathrm{H}_{2} \mathrm{CO}$ and $\mathrm{SiO}$ masers (Ginsburg et al. 2015), cloud d contains a $\mathrm{H}_{2} \mathrm{CO}$ maser, which suggests massive star formation (Immer et al. 2012), and Sgr B2 is heavily forming stars, which heats the surrounding medium. From the qualitative picture, gas and stars seem to go hand in hand, providing a rough picture that needs to be tested in more detail in order to reach the goal of a well-calibrated absolute timeline of star formation in the CMZ.

\section{Summary and Conclusions}

We described the observations and data reduction of the SWAG survey and presented the first data products. This paper covers the central $\sim 250 \mathrm{pc}$ of the $\mathrm{CMZ}$ in the Galactic Center in the six metastable ammonia inversion lines $\mathrm{NH}_{3}(1,1)$ to $(6,6)$ at a resolution of $\sim 22^{\prime \prime}(\sim 0.9 \mathrm{pc})$. The gas distribution is asymmetric with respect to the Galactic Center $\left(\operatorname{Sgr~A}^{*}\right)$ as most gas is detected at positive longitudes. We find ammonia gas in the known arching structures, especially in the dust ridge spanning from the Brick $(\mathrm{G} 0.253+0.016)$ to Sgr B2. The typical $\mathrm{NH}_{3}(3,3)$ main beam brightness is found to be on the order of a few hundred mJy beam ${ }^{-1}$ (a few K), but reaches up to $5.28 \mathrm{Jy}_{\text {beam }^{-1}}(24.8 \mathrm{~K})$ in Sgr B2. Ammonia gas at $[0, b>0]$ is oriented approximately perpendicular to the Galactic plane and shows significantly higher velocities than the rotating arc structures. The typical velocity dispersions are $5-25 \mathrm{~km} \mathrm{~s}^{-1}$, but they are affected by blending of ammonia hyperfine components and multiple emission components along the line of sight.

Fitting the hyperfine structure allows us to derive maps of line width, opacity, column density, and kinetic gas temperature. We find the line widths to be $\Delta v(\mathrm{FWHM})=$ $6-18 \mathrm{~km} \mathrm{~s}^{-1}$ with a typical value of $\sim 10 \mathrm{~km} \mathrm{~s}^{-1}$, consistent for the six ammonia species. Large areas of the CMZ are optically thin, with the more massive clouds being somewhat optically thick at $\tau<5$. Exceptions of high opacity $(\tau \gtrsim 10)$ are the Brick, several clouds surrounding Sgr B2, an extension to the $20 \mathrm{~km} \mathrm{~s}^{-1}$ cloud, and G-0.40-0.20. Ammonia column densities reach up to $N \sim 7 \times 10^{16} \mathrm{~cm}^{-2}$ in Sgr B2 and several $10^{16}$ in the massive CMZ clouds. Smaller clouds on the gas "ring" show lower column densities of $N<10^{16} \mathrm{~cm}^{-2}$.

The kinetic gas temperatures exhibit two components, a cold component of $25-50 \mathrm{~K}$ traced by $T_{12}$, and a warm component of $50-120 \mathrm{~K}$ traced by $T_{24}, T_{45}$, and $T_{36}$ where $T_{i j}$ denotes the temperature derived from ammonia states $\mathrm{NH}_{3}(\mathrm{i}, \mathrm{i})$ and $\mathrm{NH}_{3}(\mathrm{j}, \mathrm{j})$. Sgr B2 stands out as the warm component and is detected at up to $\sim 200 \mathrm{~K}$. On average over the CMZ, the cold component contributes $\sim 55 \%$ to the ammonia column density, the warm component $\sim 45 \%$. In the $20 \mathrm{~km} \mathrm{~s}^{-1}$ and $50 \mathrm{~km} \mathrm{~s}^{-1}$ clouds, the cold gas fraction is higher $(\sim 68 \%)$ than in the dust 


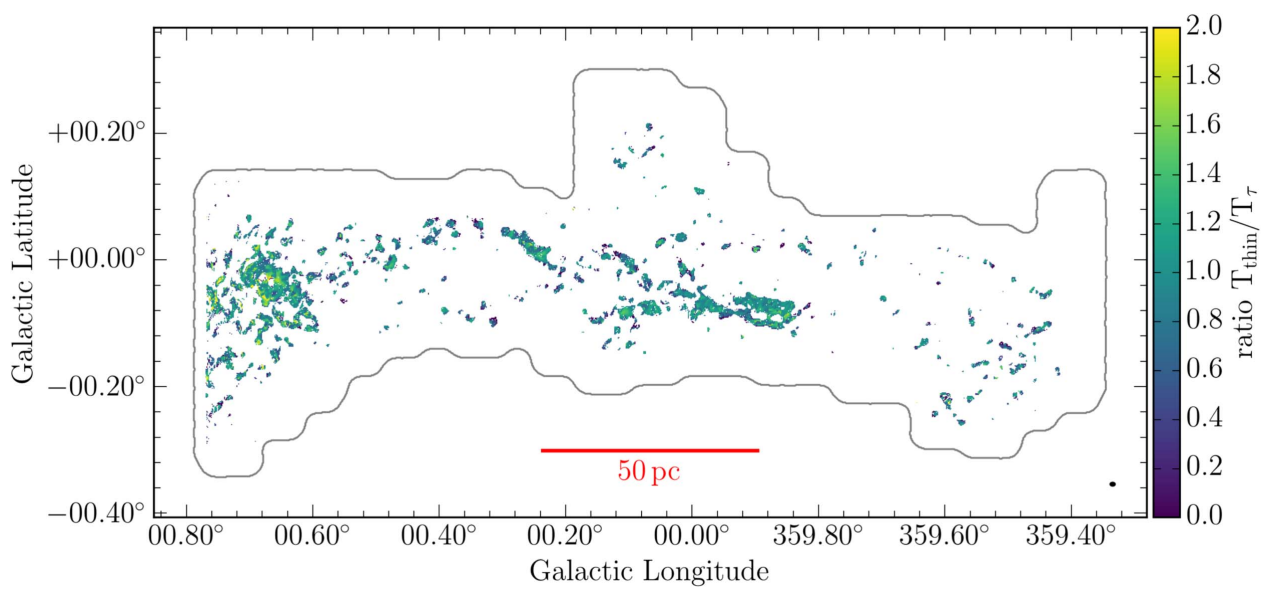

Figure 34. Ratio map $T_{\text {thin }} / T_{\tau}$ of the ammonia $T_{24}$ temperature assuming optically thin emission $\left(T_{\text {thin }}\right)$ and considering opacity effects $\left(T_{\tau}\right)$. The differences are minor (blue-green) in large areas of the map, but the temperature can be overestimated (yellow) in dense regions by factors of $\sim 2.0$. Temperatures in less dense clouds and at the edges of the fitted area are underestimated (dark blue).

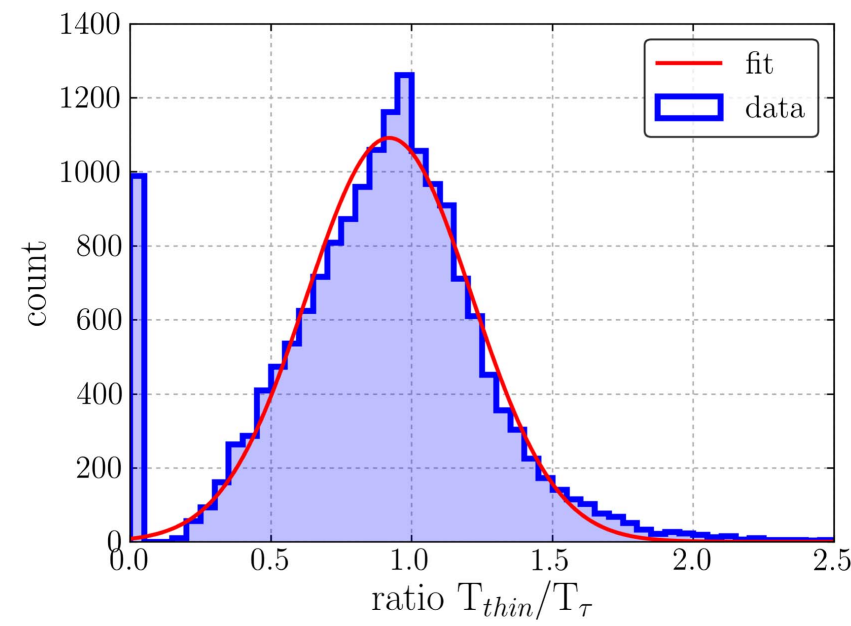

Figure 35. Histogram of the temperature ratio $T_{\text {thin }} / T_{\tau}$ between the kinetic ammonia temperature $T_{24}$ derived assuming optically thin emission and considering measured opacities (Figure 34). For $\sim 1000$ pixels at a ratio of 0.0 , the hyperfine structure could be fitted and an opacity-corrected temperature derived, although the emission is faint enough to be masked while calculating the image moment 0 on which the temperature under thin assumption is based. The inverse case (ratios of $+\infty$ ) occurs more often, but does not show up in this plot. A Gaussian fit generally describes the distribution well, but does not fit a greater amount of temperature ratios of 1.0 and 1.5-2.0. The latter is the effect of considering the opacity for optically thick emission. As lower ammonia states are typically more occupied (falling slope in Boltzmann plot) and the opacity correction increases occupation over the thin approximation $\left(N_{\tau}>N_{\text {thin }}\right)$, the temperature decreases $\left(T_{\tau}<T_{\text {thin }}\right)$ and thus $T_{\text {thin }} / T_{\tau}>1.0$.

ridge $(\sim 60 \%)$. The warm gas component is more pronounced in Sgr B2, where both cold and warm gas contribute equally ( $\sim 50 \%$ each) to the total column density. We confirm the kinetic gas temperature to be offset from the dust temperature, for which we derive offsets of $\sim 10-15 \mathrm{~K}$ for the cold component and $\sim 75-100 \mathrm{~K}$ for the warm component. Despite the offset, the median gas temperatures of both components are linearly correlated with the dust temperature.

One possibility to exploit this rich data set is given in Section 5 by statistically analyzing CMZ temperature, column density, and line width as a function of time based on the dynamical model of K15. We find evidence for a consistent, gradual heating of $52 \mathrm{~K} \mathrm{Myr}^{-1}$ in two sequences in the dust ridge and at near-side pericenter passage in the three partially independent ammonia temperature tracers $T_{24}, T_{45}$, and $T_{36}$. Near
Sgr C, a steeper trend of $\sim 100 \mathrm{~K} \mathrm{Myr}^{-1}$ is detected, whereas at far-side pericenter passage, we identify a qualitative increase of gas temperature. A hint on time-dependent evolution is also found as a decreasing sequence of column density at near-side pericenter passage, while other time ranges show no or no consistent evolution. No evolution is found in line width, but instead, variations between clouds dominate potential weaker evolutionary trends. This time dependence can be explained in the context of Longmore et al. (2013b) and K15 as being the result of tidal triggering of cloud collapse when passing pericenter. Gravitational energy released through a turbulence cascade triggered by tidal compression is potentially the source of heating near pericenter. The observed sequences of rising temperatures can result if the heat cannot be efficiently radiated away in the dense molecular clouds. Clouds downstream the triggering point continue to collapse and begin to form stars in their cores, which introduces an increasing source of heat that likely powers the second sequence of rising gas temperatures. Stacking of the identified sequences results in a weaker correlation because of the scatter of the exact collapse triggering point, i.e., clouds do not collapse at identical orbital phase. By allowing the starting point of each sequence to vary by up to $\pm 0.3 \mathrm{Myr}$, we recover a heating rate of $\sim 48 \mathrm{~K} \mathrm{Myr}^{-1}$ and estimate the triggering point to scatter by $-0.2 \mathrm{Myr}$ to +0.3 Myr.

Our work demonstrates the power of ammonia temperature measurements in inferring physical conditions. Usually a tool for investigations on small scales, we greatly expanded the spatial scale as was never done before to derive temperatures, column density, and opacity in the inner CMZ, and finally, the whole CMZ in the full SWAG survey. Despite the large area, SWAG offers high resolution, which allows statistical conclusions like the observed rising temperature sequences. The other data of SWAG, spatial as well as spectral coverage, will be presented in forthcoming papers.

H.B. acknowledges support from the European Research Council under the Horizon 2020 Framework Program via the ERC Consolidator Grant CSF-648505. J.M.D.K. gratefully acknowledges funding from the German Research Foundation (DFG) in the form of an Emmy Noether Research Group (grant number KR4801/1-1, PI Kruijssen), from the European Research Council (ERC) under the European Union's Horizon 


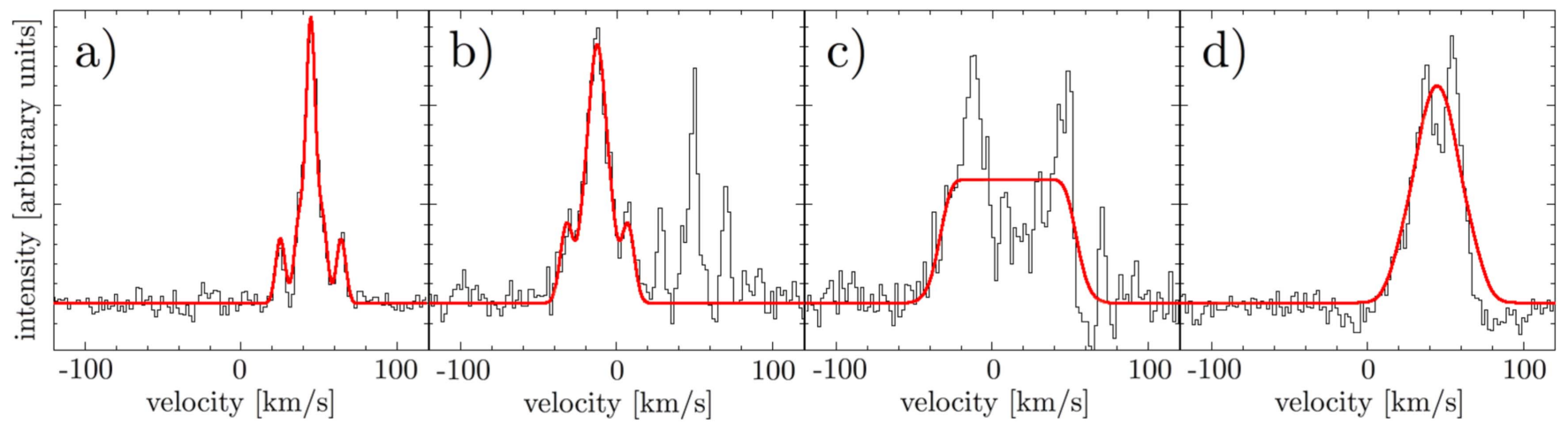

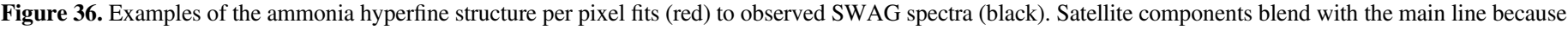

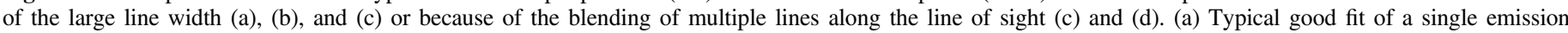

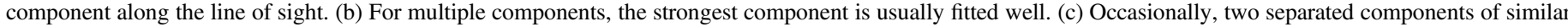

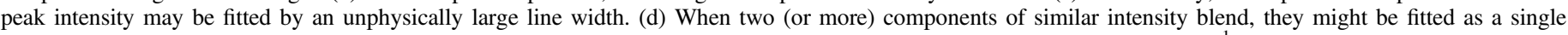

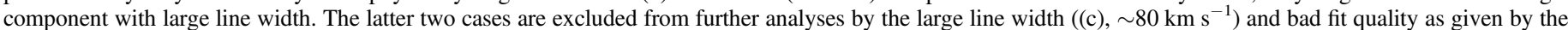
reduced $\chi^{2}(\mathrm{~d})$.

2020 research and innovation programme via the ERC Starting Grant MUSTANG (grant agreement number 714907, PI Kruijssen), and from Sonderforschungsbereich SFB 881 "The Milky Way System" (subproject P1) of the DFG. E.R. is supported by a Discovery Grant from NSERC of Canada. T.P. acknowledges support from the Deutsche Forschungsgemeinschaft, DFG via the SPP (priority program) 1573 Physics of the ISM. The National Radio Astronomy Observatory is a facility of the National Science Foundation operated under cooperative agreement by Associated Universities, Inc.

Software: CASA (McMullin et al. 2007), CLASS (http:// www.iram.fr/IRAMFR/GILDAS), miriad (Sault et al. 1995), APLpy (Robitaille \& Bressert 2012).

Facility: ATCA(CABB).

\section{Appendix A SWAG Data Products}

\section{A.1. Channel Maps}

In Figures 22-26, we present the channel maps of $\mathrm{NH}_{3}$ $(1,1),(2,2),(4,4),(5,5)$, and $(6,6)$ as discussed in Section 3.1. The channel maps of $\mathrm{NH}_{3}(3,3)$ are shown in Figure 5.

\section{A.2. Sample Spectra}

In Figure 27, we present sample spectra toward the Brick for $\mathrm{NH}_{3}(1,1)$ to $(6,6)$.

\section{A.3. Moment Maps}

Figures 28-32 show the moment maps of $\mathrm{NH}_{3}(1,1),(2,2)$, $(4,4),(5,5)$, and $(6,6)$. Their generation from the respective data cubes is explained in Section 3.3.

\section{Appendix B Cleaning Depth in MOSMEM}

Version 1.6 of mosmem as of 2015 contained $^{31}$ a bug that did not correctly scale the rms noise when emission is found, which resulted in accumulation of the background flux density level and a non-constant baseline in spectra (M. Wieringa, private

\footnotetext{
${ }^{31}$ As of 2016 August, this bug is fixed and mosmem works as expected.
}

communication). The strength of this effect depends on the amount of cleaned flux density and thus on the number of iterations. Tests on line bearing and line-free channels of SWAG data showed that for up to $\sim 50$ iterations, no measurable effects occur, while noticeable spectral slopes of tens of mJy beam ${ }^{-1}$ over the spectral range arise when $\gtrsim 100$ iterations are used, and very high iteration counts of 250-500 result in multiple sudden unpredictable jumps of $30-80 \mathrm{mJy}^{\text {beam }}{ }^{-1}$ in the spectra. These observations imply that the flux offsets are inherited from one channel to the next in the order of processing. Channels containing large amounts of emission (e.g., ammonia lines) would need about 150-200 iterations for mosmem to converge and thus still contain noticeable amounts of uncleaned sidelobe structure. The applied restriction to 50 iterations in the generation of SWAG maps therefore results in maps that are not affected by the software bug at the cost of remaining undeconvolved flux.

\section{Appendix C The Ammonia Thermometer}

Ammonia is a good thermometer because of its inversion lines, which cover rotational temperatures from close to absolute zero to thousands of Kelvin when the rotational quantum number $J$ increases. Its hyperfine structure allows directly calculating opacities, which are needed in order to derive correct temperatures without the need for a background source (on-off technique). The simplest approach in deriving column densities and subsequently temperatures is to assume optically thin emission, i.e., use moment-0 maps (Figure 7). As this technique cannot disentangle hyperfine satellite lines or multiple emission sources along the line of sight, it is problematic for ammonia and in the GC. Therefore, we fit the ammonia spectra to resolve hyperfine lines and complex emission. A comparison of these two methods for temperature deriving is given in Section C.4.1.

\section{C.1. Rotational Temperature and Boltzmann Plot}

The occupation of different rotational levels follows a Boltzmann distribution, and the ratio of two column densities therefore becomes a function of temperature (Goldsmith \& Langer 1999). For a state of energy $E$ with excitation temperature $T_{\mathrm{ex}}$ of the system, the probability of the system 


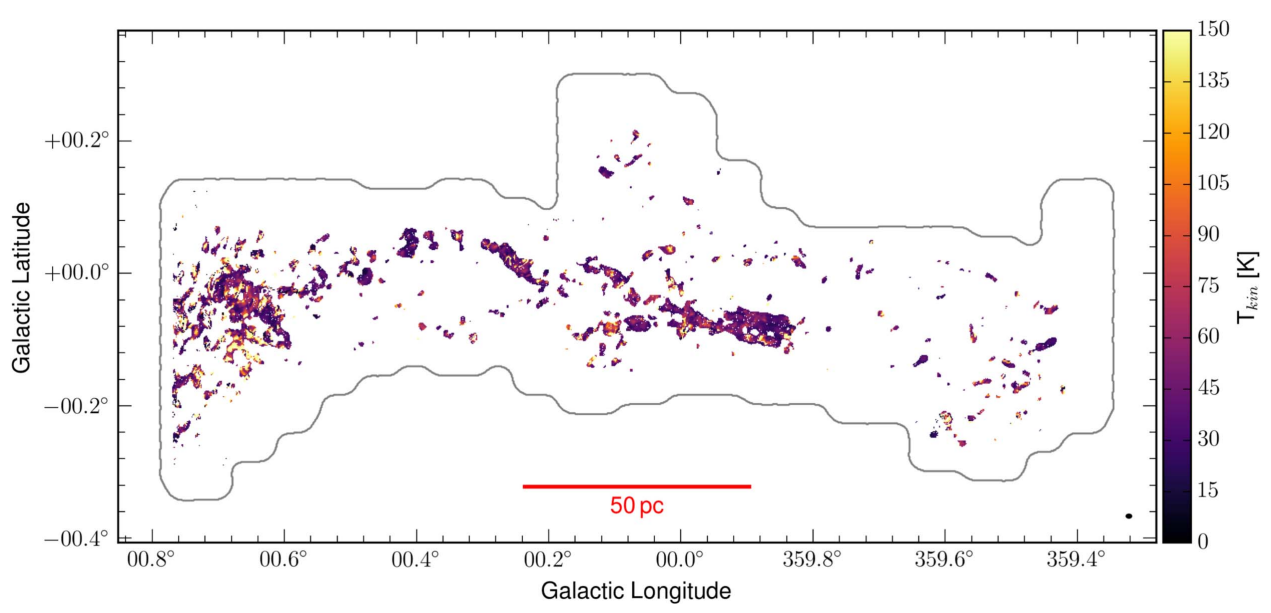

Figure 37. $T_{12}$

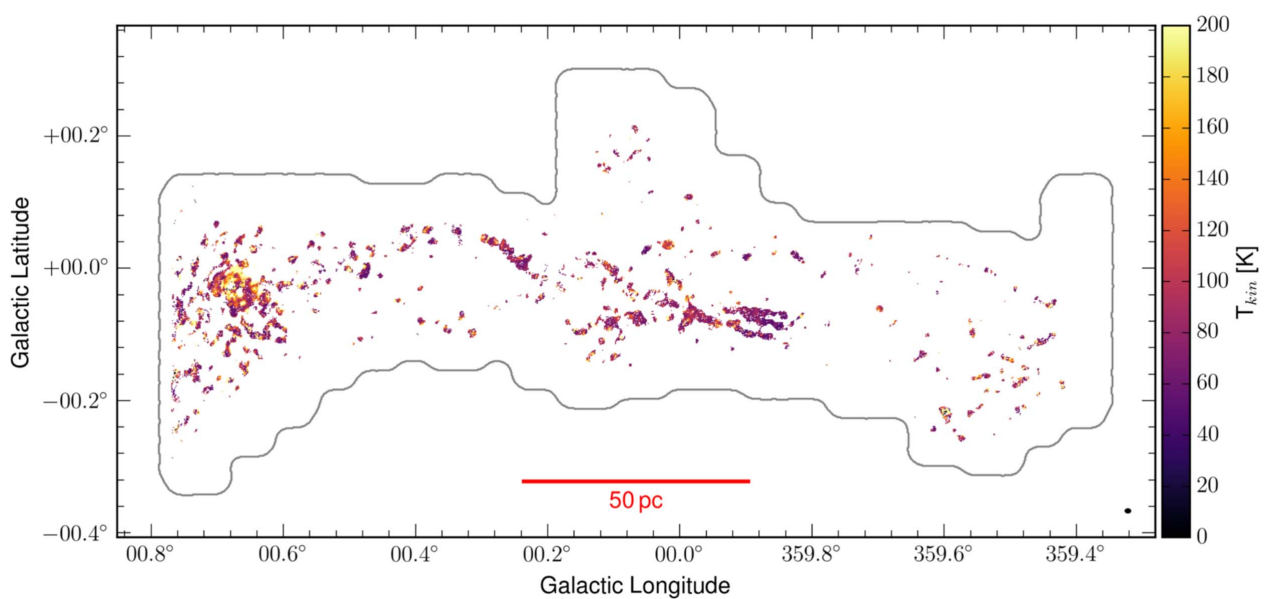

Figure 38. $T_{45}$

to be in that state is given by

$$
p \propto \exp \frac{E}{k_{\mathrm{B}} T_{\mathrm{ex}}},
$$

where $k_{\mathrm{B}}=1.38 \times 10^{-23} \mathrm{~J} / \mathrm{K}$ is the Boltzmann constant. For two metastable inversion lines with rotational numbers $J=K$, the relative probability is called Boltzmann factor and depends only the energy difference $\Delta E$. For rotational states, the temperature $T_{\mathrm{ex}}$ is accordingly called rotational temperature $T_{J J^{\prime}}$ for states $J, J^{\prime}$ after considering the correct conversion. The probability of being in a certain state can be translated into the number of particles in that state, which can be approximated by column densities because only the Boltzmann factor (relative ratio) is needed instead of absolute values. Therefore, the ratio of column density $N_{\mathrm{u}}$ in the upper rotational state $u$ with $J$ or $J^{\prime}$ can be expressed as

$$
\frac{N_{u}^{\prime}\left(J^{\prime}, J^{\prime}\right)}{N_{u}(J, J)}=\frac{g\left(J^{\prime}\right)}{g(J)} \frac{2 J^{\prime}+1}{2 J+1} \exp \left(\frac{-\Delta E}{T_{J J^{\prime}}}\right)
$$

with relative weight factors of $2 J+1$ and statistical weights $g$ that follow from quantum mechanical considerations as derived in Schilke (1989). It must be taken into account that ammonia can exist in two different configurations of the hydrogen atom nuclear spin, all spins parallel, or one spin antiparallel to the other two. The configurations differ in energy and thus give rise to two ladders of ammonia states, ortho for rotational states $J=0,3,6, \ldots$ (all spins parallel, statistical weight $g=2$ ) and para for $J=1,2,4,5, \ldots$ (one spin antiparallel, $g=1$ ). Equation (3) can be rewritten as

$$
\underbrace{\ln \left(\frac{N_{u}^{\prime}\left(J^{\prime}, J^{\prime}\right)}{g^{\prime}\left(2 J^{\prime}+1\right)}\right)}_{y^{\prime}}-\underbrace{\ln \left(\frac{N_{u}(J, J)}{g(2 J+1)}\right)}_{y}=-\underbrace{\frac{\Delta \mathrm{E}}{k_{\mathrm{B}} T_{\mathrm{rot}, \mathrm{JJ}^{\prime}}}}_{\Delta x / T_{\mathrm{rot}, \mathrm{JJ}}},
$$

which is an equation of simple linear form for the inverse temperature with slope $m$,

$$
m=\frac{\Delta y}{\Delta x}=\frac{y^{\prime}-y}{\Delta x} .
$$

The corresponding plot is called Boltzmann plot, with energy above ground state on the $x$-axis and $y$-axis values derived from measured column densities. For convenience, $y$ is given in logarithms of base 10 instead of $e$ as

$$
y=\log _{10}\left(\frac{N(J, J)}{g(J)(2 J+1)}\right)
$$




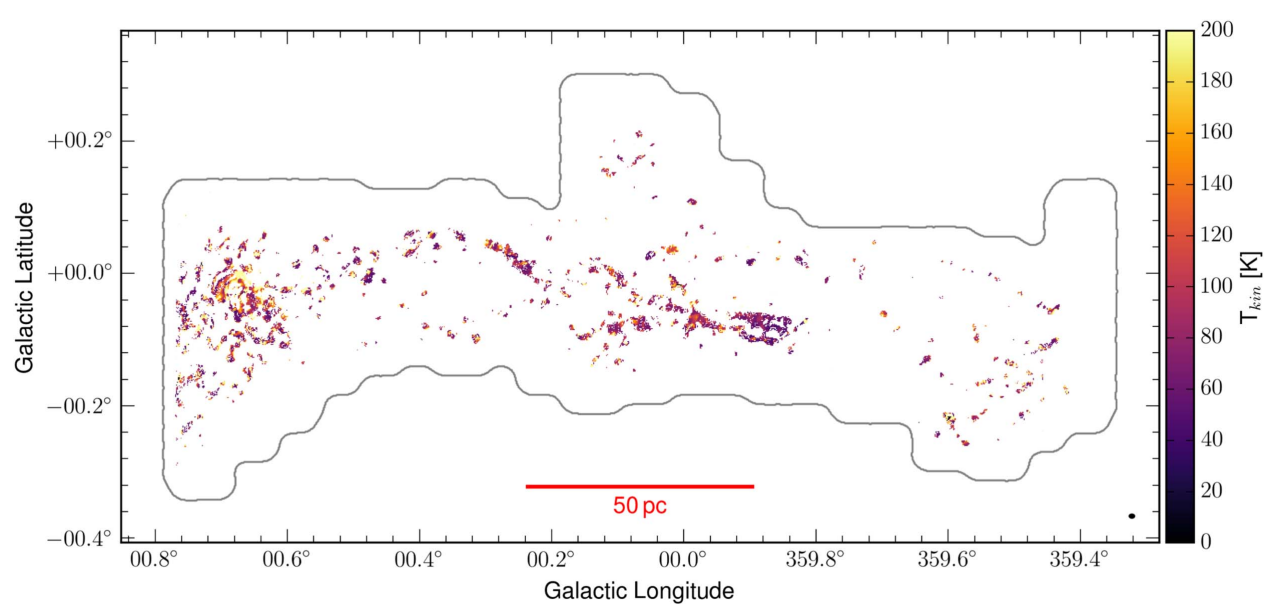

Figure 39. $T_{36}$

which leads to a correction factor of $\log _{10}(e)$ when calculating temperatures,

$$
T_{\mathrm{rot}, \mathrm{JJ}^{\prime}}=\frac{-\log _{10}(e)}{m} .
$$

Overall, this means the rotational temperature of ammonia gas can be derived from the slope between two measurements of column density for the known state energies.

\section{C.2. Excitation Temperature}

For radio frequencies and the expected ISM temperatures $(T[\mathrm{~K}] \gtrsim 10 \mathrm{~K})$, the Rayleigh-Jeans approximation for blackbody emission holds because $\exp \left(-\frac{h \nu}{k_{\mathrm{B}} T}\right) \approx 1-\frac{h \nu}{k_{\mathrm{B}} T}$. With intensities expressed as line temperatures and considering the limited beam size of a telescope, radiative transfer can be expressed as

$$
T_{L}(\nu)=\eta_{f} T_{\mathrm{ex}}\left(1-e^{-\tau}\right)+\eta_{f} T_{\mathrm{BG}} e^{-\tau}+\left(1-\eta_{f}\right) T_{\mathrm{BG}}
$$

in the notation of Schilke (1989). $T_{L}$ is the observed line temperature that results from source emission with excitation temperature $T_{\mathrm{ex}}$ and background $T_{\mathrm{BG}}$ that both pass through a medium with opacity $\tau$. The beam filling factor $\eta_{f}$ denotes the fractional area of the beam filled by the source on the sky. For completeness, it will be dragged along in further equations, but set to unity in all calculations as this is expected to be approximately given for the beam size of SWAG $(\sim 0.9 \mathrm{pc})$ and the extended emission detected $\left(>20^{\prime \prime}\right)$.

Solving for $T_{\mathrm{ex}}$ results in

$$
T_{\mathrm{rot}, \mathrm{JJ}^{\prime}}=T_{\mathrm{ex}}=\frac{T_{L}}{f\left(1-e^{-\tau}\right)}+T_{\mathrm{BG}} .
$$

The background temperature $T_{\mathrm{BG}}$ is set by the cosmic microwave background (CMB) at $2.7 \mathrm{~K}$.

\section{C.3. Column Density}

In order to calculate rotational temperatures via Equation (7), the column densities of two states need to be known, which are given by

$$
N(J, K)=\frac{3 h}{8 \pi^{3}} \frac{J(J+1)}{K^{2}} \frac{1}{\mu^{2}} \frac{k_{\mathrm{B}}}{h \nu_{J K}} \int T_{\mathrm{ex}} d \nu,
$$

with the Planck constant $h$, the Boltzmann constant $k_{B}$, the angular momentum $J$, the projected angular momentum $K$, the ammonia dipole moment $\mu$, the frequency $\nu$ of the transition $J \rightarrow J^{\prime}$, and the excitation temperature $T_{\mathrm{ex}}$ integrated over the velocity $d v$.

The integral is solved differently when assuming optically thin emission or considering opacities.

\section{C.3.1. Column Density in the Optically Thin Limit}

Assuming optically thin emission, column densities can simply be derived from observed intensities with the appropriate scaling factors because every detected photon directly corresponds to one emitting molecule. Following Ott et al. (2005, 2014), Mills \& Morris (2013), whose work is based on Hüttemeister et al. (1993, 1995), and Mauersberger et al. (2003), the column density $N$ in state $(J, K)$ depends on the electric dipole moment $\mu$ in Debye and on the line brightness temperature $T_{\mathrm{mb}}$ as

$$
\begin{aligned}
& N(J, K)\left[\mathrm{cm}^{-2}\right]=1.6698 \cdot 10^{14} \cdot \frac{J(J+1)}{K^{2}} \\
& \times \frac{1}{\mu^{2}\left[D^{2}\right] \cdot \nu[\mathrm{GHz}]} \int_{\text {line }} T_{\mathrm{mb}} d \nu\left[\mathrm{K} \mathrm{km} \mathrm{s}^{-1}\right] \\
& =\frac{7.77 \times 10^{13}}{\nu[\mathrm{GHz}]} \frac{J(J+1)}{K^{2}} \int_{\text {line }} T_{\mathrm{mb}} d \nu\left[\mathrm{K} \mathrm{km} \mathrm{s}^{-1}\right]
\end{aligned}
$$

Equation (11) lists the general formula for any symmetric top molecule with dipole moment $\mu$, whereas Equation (12) already includes $\mu=1.472 \mathrm{D}$ for ammonia and shows the form given in the literature.

The datacube structure in discrete channels of width $\Delta v_{\text {chan }}$ simplifies the integration in velocity over the spectral line to a summation of spectral channels, which in turn is just the definition of moment-0 flux $F_{\mathrm{mom} 0}=\int T_{\mathrm{mb}} d v=\sum T_{\mathrm{mb}} \Delta v_{\text {chan, }}$,

$$
\begin{aligned}
& N(J, K)\left[\mathrm{cm}^{-2}\right]=1.6698 \cdot 10^{14} \frac{J(J+1)}{K^{2}} \\
& \cdot \frac{1}{\mu^{2}\left[\mathrm{D}^{2}\right] \cdot \nu[\mathrm{GHz}]} \cdot \sum_{\text {line }} T_{i} \Delta v_{\text {chan }}\left[\mathrm{K} \mathrm{km} \mathrm{s}^{-1}\right],
\end{aligned}
$$




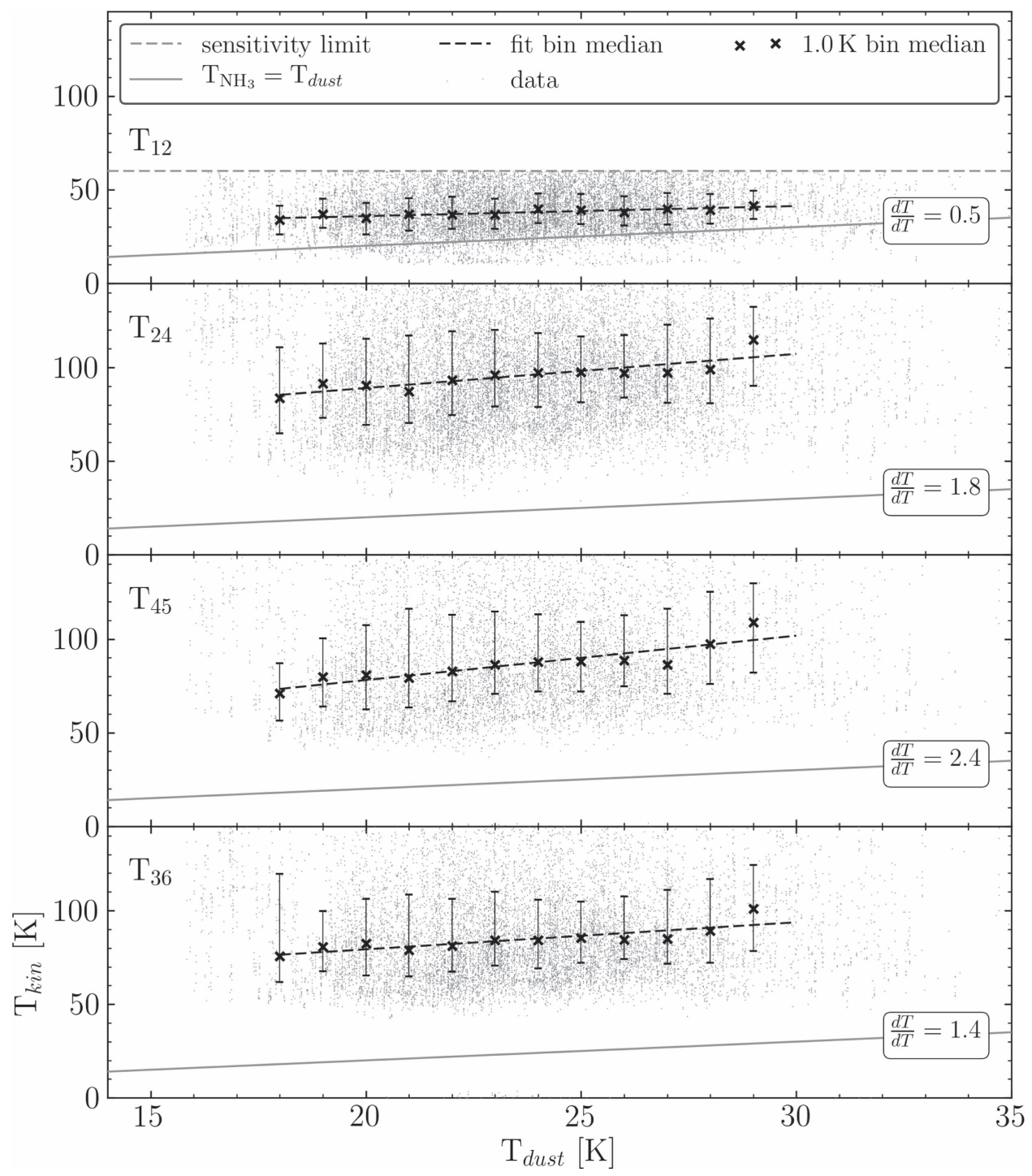

Figure 40. Correlation of the Hi-Gal dust temperature (Molinari et al. 2011) and the kinetic ammonia gas temperature as in Figure 17. The temperature estimate is labeled in the top left corner of each panel. Panels are zoomed to display the same range to allow a direct comparison.

$$
=\frac{7.77 \times 10^{13}}{\nu[\mathrm{GHz}]} \frac{J(J+1)}{K^{2}} \mathrm{~F}_{\mathrm{mom} 0}\left[\mathrm{~K} \mathrm{~km} \mathrm{~s}^{-1}\right],
$$

\section{C.3.2. Column Densities Considering Opacity}

By solving the radiative transfer equation, it is possible to derive an expression for the column density without assuming high or low optical depth because an exact solution for the relation of $T_{\mathrm{ex}}\left(T_{J J^{\prime}}\right.$ for rotational states) and observed brightness temperature $T_{\mathrm{mb}}$ is known (Schilke 1989).

The derivation starts again from the general approach in Equation (10), but then solves the integral over a spectral line of Gaussian shape (peak $T_{L}$ and FWHM $\Delta v_{\text {int }}$ ) with excitation temperature as in Equation (9). The integral over a single hyperfine component thus is given by

$$
\mathrm{A}=\frac{\sqrt{\pi}}{2 \sqrt{\ln 2}} \cdot T_{L} \cdot \Delta v_{\mathrm{int}}
$$

and the velocity integrated temperature becomes

$$
\begin{aligned}
& \int T_{\text {ex }} d v=\frac{\sqrt{\pi}}{2 \sqrt{\ln 2}} \Delta v_{\text {int }} \frac{T_{\text {ex }} \tau}{f_{J}}, \\
& =\frac{\sqrt{\pi}}{2 \sqrt{\ln 2}} \Delta v_{\text {int }} \frac{T_{L} \tau}{f f_{J}\left(1-e^{-\tau}\right)},
\end{aligned}
$$

$T_{L}$ denotes the line peak intensity and must not be confused with the integrated line intensity $T_{\mathrm{mb}}$ we used before. $\Delta v_{\text {int }}$ stands for the fitted FWHM of a single hyperfine component.

Calculating all numerical factors and logically rearranging the parts to match Equation (11)-(14) results in

$$
\begin{aligned}
N(J, K)= & 1.77745 \cdot 10^{14} \mathrm{~cm}^{-2} \cdot \frac{J(J+1)}{K^{2}} \\
& \times \frac{\Delta v_{\mathrm{int}}\left[\mathrm{km} \mathrm{s}^{-1}\right]}{(\mu[\mathrm{D}])^{2} \nu[\mathrm{GHz}]} \frac{\tau T_{L}[\mathrm{~K}]}{f_{J}\left(1-e^{-\tau}\right)} .
\end{aligned}
$$

The pre-factor of $1.77745 \cdot 10^{14}$ applies when the beam filling factor is set to unity, line width $\Delta v_{\text {int }}$ is given in $\mathrm{km} \mathrm{s}^{-1}$, 


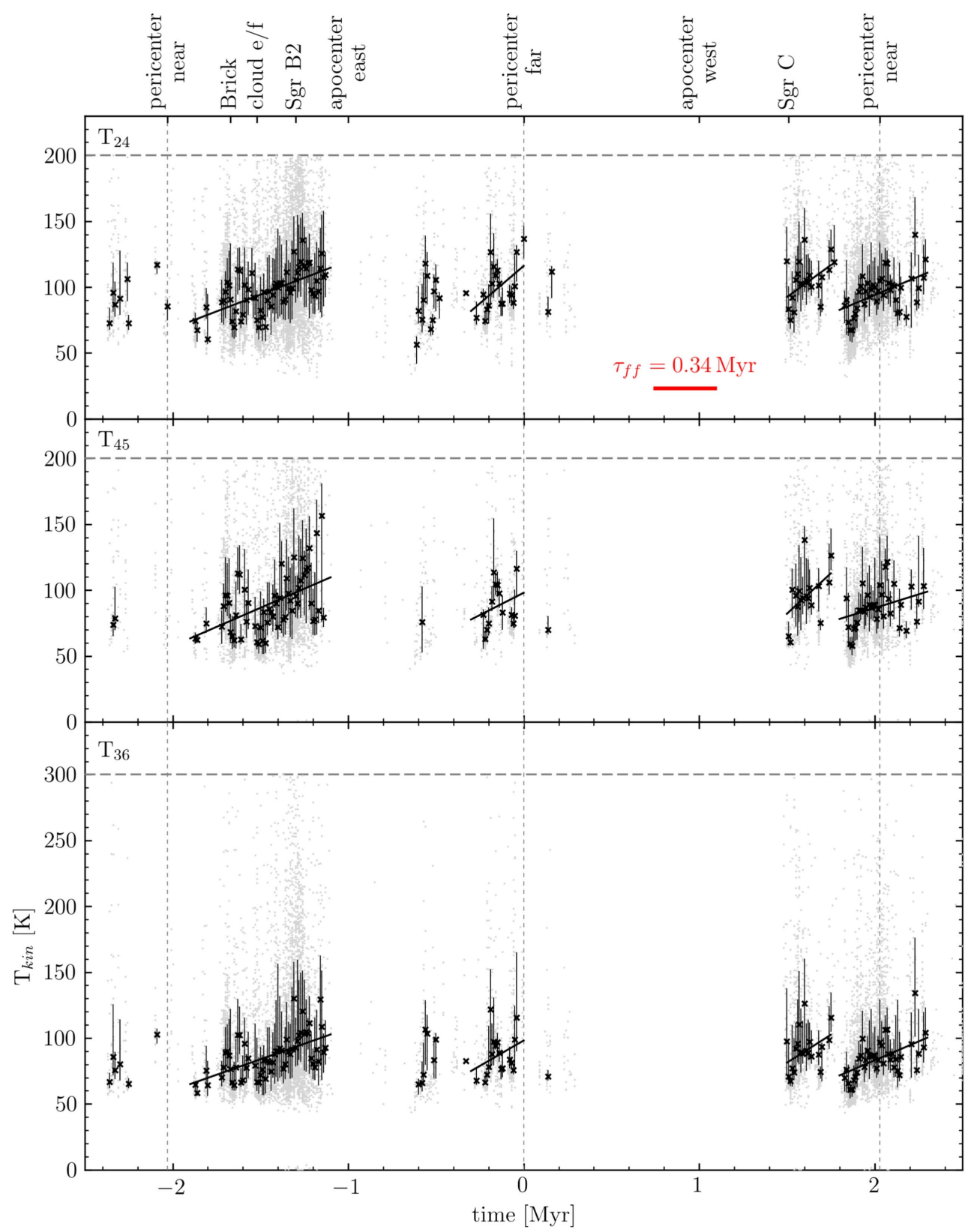

Figure 41. Kinetic ammonia temperature as a function of time since far-side pericenter passage (bottom $x$-axis; the top $x$-axis shows important dynamical points in the K15 model and massive molecular clouds). The temperature tracer is given in the top left corner of each panel. A horizontal dashed gray line denotes the sensitivity limit of the conversion from rotational into kinetic temperature. Vertical dashed lines highlight the occurrence of pericenter passages. Measurements for individual pixels (light gray points) are overlaid with black crosses denoting medians in bins of $0.01 \mathrm{Myr}$ and error intervals that include $50 \%$ of the data in each bin.

dipole moment $\mu$ in Debye, frequency of the inversion line in $\mathrm{GHz}$ and line peak intensity in Kelvin. $f_{\mathrm{J}}$ is a factor to correct the fitted opacity $\tau$ for its line profile and is basically the ratio of flux in the central hyperfine component (see Schilke 1989, for details). Table 3 lists $f_{J}$ for $\mathrm{NH}_{3}(1,1)$ to $(6,6)$ for five hyperfine components per line, whereof the central ("component $0^{\prime \prime}$ ) enters Equation (18).

Figure 33 shows the column density distribution of the upper state $N_{u}$ of $\mathrm{NH}_{3}(3,3)$ as an example.

\section{C.3.3. Total Ammonia Column Density}

Note that Equations (18) and (12) and Figure 33 give the column density of ammonia in the lower transition state only. The total column density of the state $(J, K)$ must also take the molecules in the upper transition state into account. In thermal equilibrium, the column densities of both states are related by a Boltzmann factor

$$
\begin{aligned}
& N(J, K)=N_{l}(J, K)\left(1+\exp \left(-\frac{h \nu}{k_{\mathrm{B}} T}\right)\right) \\
& \simeq 2 \times N_{l}(J, K) \quad \text { for } T \gg \frac{h \nu}{k_{\mathrm{B}}} .
\end{aligned}
$$

In the ground state $(J=0)$, the inversion states are degenerate and no transition can be observed, which means that the column density $N_{00}$ cannot be derived directly but needs to be extrapolated from low metastable states. As the slope in a typical Boltzmann plot (excitation state-scaled 


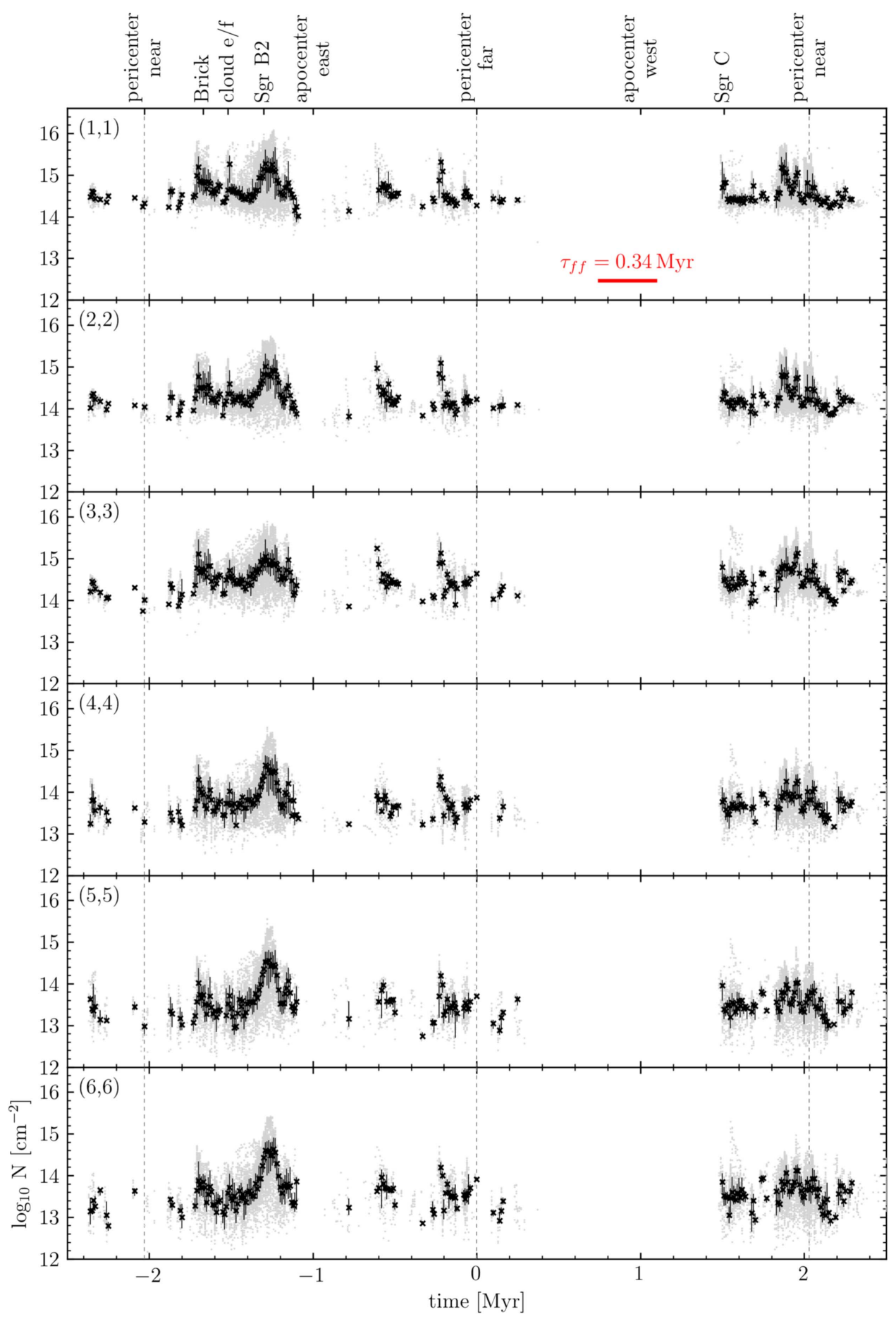

Figure 42. Ammonia column density as a function of time since far-side pericenter passage (bottom $x$-axis; the top $x$-axis shows important dynamical points in the K15 model and massive molecular clouds). The ammonia species is given in the top left corner of each panel. Vertical dashed lines highlight the occurrence of pericenter passages. Measurements for individual pixels (light gray points) are overlaid with black crosses denoting medians in bins of $0.01 \mathrm{Myr}$ and error intervals that include $50 \%$ of the data in each bin.

column density versus excitation temperature) of ammonia emission of the GC starts to flatten around $J=4-6$, the best estimate for $N_{00}$ is derived from the slope between measurements at $J=1$ and $J=2$, i.e., rotational temperature $T_{12}$. If the ortho/para ammonia abundance ratio is not known, the simplest approach is to assume occupation by thermal equilibrium of states $J=0,1,2$ at temperature $T_{12}$, hence to extrapolate $N_{00}$ according to Equation (21) (Ungerechts et al. 1986). The factor $23.2 \mathrm{~K}$ is given by the energy difference of $\mathrm{NH}_{3}(0,0)$ and $\mathrm{NH}_{3}(1,1)$,

$$
N_{00}=\frac{1}{3} \exp \left(\frac{23.2 \mathrm{~K}}{T_{\text {kin, } 12}}\right) N_{11} .
$$

The "total" column density of ammonia is then derived by summing the six directly measured column densities 


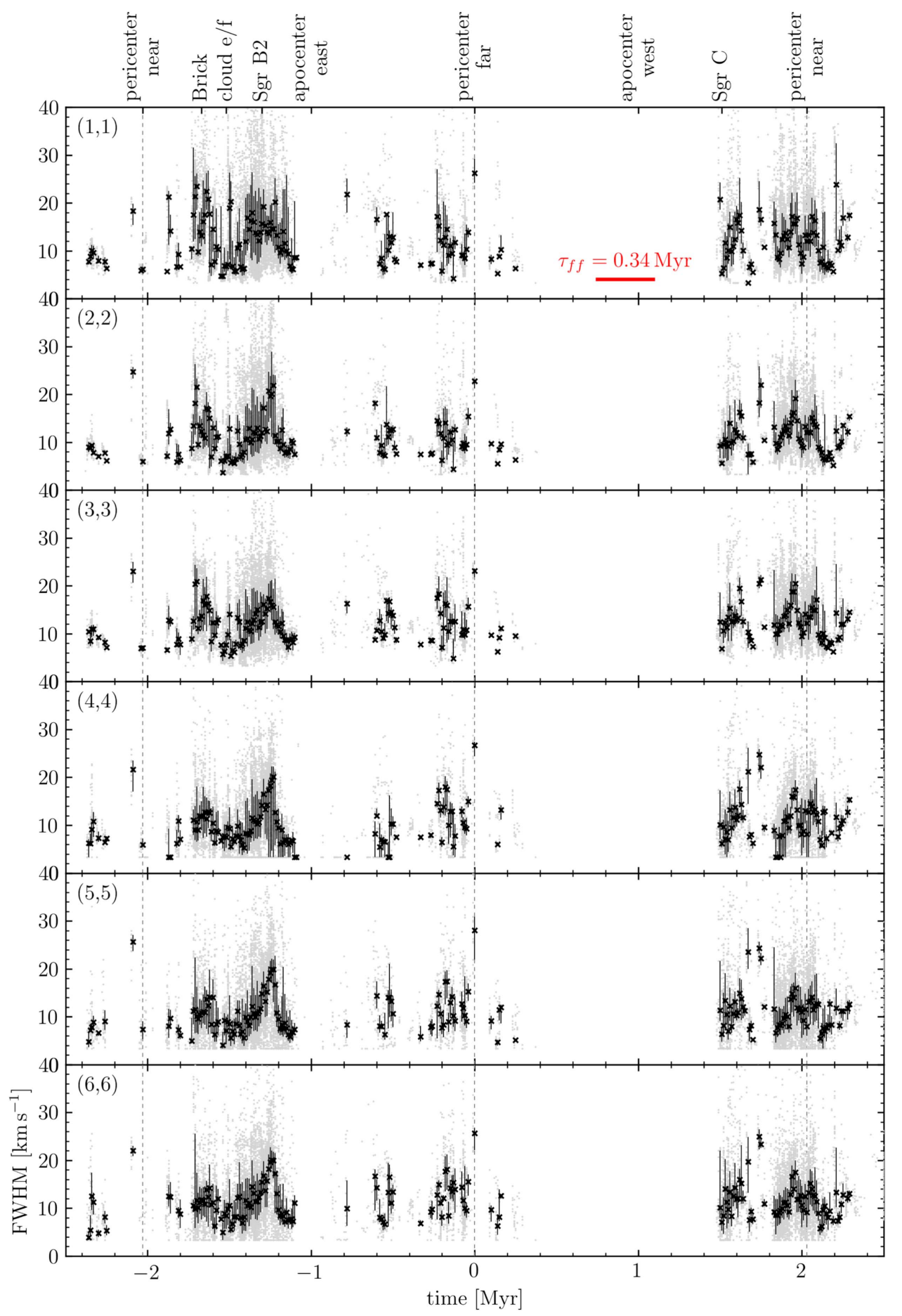

Figure 43. Ammonia line width as a function of time since far-side pericenter passage (bottom $x$-axis; the top $x$-axis shows important dynamical points in the K15 model and massive molecular clouds). The ammonia species is given in the top left corner of each panel. Vertical dashed lines highlight the occurrence of pericenter passages. Measurements for individual pixels (light gray points) are overlaid with black crosses denoting medians in bins of 0.01 Myr and error intervals that include $50 \%$ of the data in each bin.

$\left(N=N_{l}+N_{u}\right)$ for states $J=1, \ldots, 6$ and the extrapolated $J=0$ column density. In thermal equilibrium, higher states are increasingly less populated and can thus be approximated to contribute negligibly to the total column density for low temperatures. Equation (22) uses these assumptions, i.e., does not contain the contribution of ammonia in rotation states higher than $J=6$ and consequently must be understood as a lower limit. How close this limit is to the real ammonia column density can roughly be estimated by the ratio $N_{00} / N_{66}$, which in SWAG typically exceeds 10 with only few very hot clouds such as Sgr B2 that contain relevant amounts of ammonia in higher excitation states, as was also found by Mills \& Morris (2013). The assumption of thermal equilibrium, which is required to extrapolate the total column density, does not hold in all CMZ clouds, whereas the lower limit is supported by direct measurements and thus is more robust. 
The total column density of ammonia (defined to include emission up to $J=6$ ) is thus given by

$$
\begin{aligned}
N_{\text {tot }}= & {\left[\frac{1}{3} \exp \left(\frac{23.2 \mathrm{~K}}{T_{12, \text { kin }}}\right)+1\right] N_{11} } \\
& +N_{22}+N_{33}+N_{44}+N_{55}+N_{66} .
\end{aligned}
$$

\section{C.4. Rotational to Kinetic Temperature Conversion}

The rotational temperature is always lower than the kinetic temperature, with negligible difference at low values. For increasing temperature, the deviation also increases and must be taken into account. Reasons for this behavior are the increasing number of energetically available non-metastable levels for depopulation of higher metastable states and radiative decay (Walmsley \& Ungerechts 1983). LVG radiative transfer models allow deriving approximations to the conversion of rotational to kinetic temperature. Ott et al. (2011) calculated LVG grids for temperatures up to $500 \mathrm{~K}$ for metastable ammonia species up to $(6,6)$ and derived exponential functions for the rotational-to-kinetic temperature conversion. These fits agree with the modeled curves within less than $5 \%$ for the temperature measures calculated in this work, which are $T_{12}$, $T_{24}, T_{45}$, and $T_{36}$, denoting the kinetic temperature $T_{i j}$ derived from $\mathrm{NH}_{3}(\mathrm{i}, \mathrm{i})$ and $\mathrm{NH}_{3}(\mathrm{j}, \mathrm{j})$. Table 4 lists the conversion functions as partially published in Ott et al. (2011) and Gorski et al. (2017). Toward high kinetic temperatures, the conversion flattens out, which decreases the ability to correctly discern temperatures. Especially the temperature measurements of low ammonia states are affected, effectively setting an upper limit above which the tracer becomes unreliable as even small changes in $T_{\text {rot }}$ by random errors translate into substantial changes in $T_{\text {kin. }}$. A cutoff is thus applied to all rotational temperatures before conversion to exclude high values that cannot be reliably translated into kinetic temperatures.

With the $T_{\text {kin }}-T_{\text {rot }}$ conversion in hand, all further mentions of temperature are kinetic temperatures labeled $T_{i j}$ if not explicitly stated otherwise, e.g., by the subscript $T_{\text {rot }, i j}$.

\section{C.4.1. Optical Depth Effects on Temperature}

As described in Appendix C, temperatures can also be calculated assuming optically thin emission, as is often done (in extra-galactic contexts), with the advantage of being able to derive temperatures over a larger area. From the fitted opacities (Figure 14), it is already known that this assumption is not satisfied in a significant portion of the map. The practical differences for derived temperatures are shown in Figure 34, which plots the ratio $T_{\text {thin }} / T_{\tau}$ of the kinetic temperature $T_{24}$ derived under the assumption of optically thin emission and considering fitted opacities. The differences are minor in large areas of the map, but the temperature can be overestimated by $T_{\text {thin }}$ in dense regions by factors of up to 1.6 in Sgr B2. Temperatures in less dense clouds and at the edges of the fitted area are typically underestimated by factors of $\lesssim 2$. This can also be seen in a histogram of the map (Figure 35) as an increase in ratios $>1.0$ over what is expected from statistical, normally distributed errors (Gaussian fit). In an optically thick molecular cloud, not all emission can be detected because of self-absorption and scattering, as would be the case in an optically thin cloud. The derived column densities thus increase when opacity is considered. The temperature is derived from the inverse slope of the column density as a function of molecular energy level, which results in lower temperatures for two conditions: when the opacity is considered in calculating the column density, and when the opacity decreases for higher states, which typically is the case. Given that the opacity typically decreases for higher states, the effect on column density is stronger for lower $J$ transitions. As the temperature is derived from the inverse slope of the column density as a function of molecular energy level, considering the opacity results in lower temperatures.

\section{Appendix D \\ Details on Hyperfine Structure Fitting in CLASS}

Figure 36 shows the typical fit quality and two examples of failed fits which are excluded from the further analysis. Table 5 lists the statistics of a pixel-by-pixel fitting.

\section{Appendix E Further Temperature Maps}

Maps of the kinetic temperature tracers T12, T45, and T36 are shown in Figures 37-39, respectively.

\section{Appendix F \\ Further Temperature Comparisons}

Figure 40 shows the correlation between the four tracers of ammonia gas temperature and dust temperature.

\section{Appendix G \\ Further Sequence Plots}

Figures 41-43 show the orbital evolution of ammonia gas temperature, column density and line width for the four temperature tracers and six ammonia lines.

\section{ORCID iDs}

Nico Krieger (iD https://orcid.org/0000-0003-1104-2014 Jürgen Ott (ib https://orcid.org/0000-0001-8224-1956 Henrik Beuther (iD https://orcid.org/0000-0002-1700-090X Fabian Walter (i) https://orcid.org/0000-0003-4793-7880 David S. Meier (i) https://orcid.org/0000-0001-9436-9471 Elisabeth A. C. Mills (ib https://orcid.org/0000-00018782-1992

Yanett Contreras (iD https://orcid.org/0000-0002-6388-3635 Adam Ginsburg (i) https://orcid.org/0000-0001-6431-9633 Christian Henkel (iD https://orcid.org/0000-0002-7495-4005 James Jackson (10) https://orcid.org/0000-0002-3466-6164 Steven Longmore (iD https://orcid.org/0000-0001-6353-0170 Mark R. Morris (iD https://orcid.org/0000-0002-6753-2066 Erik Rosolowsky (i) https://orcid.org/0000-0002-5204-2259 Hiroko Shinnaga (iD https://orcid.org/0000-0001-9407-6775 Qizhou Zhang (iD https://orcid.org/0000-0003-2384-6589

\section{References}

Ao, Y., Henkel, C., Menten, K. M., et al. 2013, A\&A, 550, A135 Bally, J., Aguirre, J., Battersby, C., et al. 2010, ApJ, 721, 137 Barnes, A. T., Longmore, S. N., Battersby, C., et al. 2017, MNRAS, 469, 2263 Betz, A. L., McLaren, R. A., \& Spears, D. L. 1979, ApJL, 229, L97

Bigiel, F., Leroy, A., Walter, F., et al. 2010, AJ, 140, 1194

Boehle, A., Ghez, A. M., Schödel, R., et al. 2016, ApJ, 830, 17

Churazov, E., Khabibullin, I., Ponti, G., \& Sunyaev, R. 2017, MNRAS, 468,165

Clark, P. C., Glover, S. C. O., Ragan, S. E., Shetty, R., \& Klessen, R. S. 2013, ApJL, 768, L34 
Clavel, M., Terrier, R., Goldwurm, A., et al. 2013, A\&A, 558, A32 Federrath, C., Rathborne, J. M., Longmore, S. N., et al. 2016, ApJ, 832, 143 Ferrière, K., Gillard, W., \& Jean, P. 2007, A\&A, 467, 611 Ginsburg, A., Henkel, C., Ao, Y., et al. 2016, A\&A, 586, A50 Ginsburg, A., Walsh, A., Henkel, C., et al. 2015, A\&A, 584, L7 Goldsmith, P. F., \& Langer, W. D. 1999, ApJ, 517, 209

Gorski, M., Ott, J., Rand, R., et al. 2017, ApJ, 842, 124

Güsten, R., Walmsley, C. M., \& Pauls, T. 1981, A\&A, 103, 197

Henkel, C., Braatz, J. A., Menten, K. M., \& Ott, J. 2008, A\&A, 485, 451

Henshaw, J. D., Longmore, S. N., \& Kruijssen, J. M. D. 2016a, MNRAS, 463, L122

Henshaw, J. D., Longmore, S. N., Kruijssen, J. M. D., et al. 2016b, MNRAS, 457, 2675

Ho, P. T. P., Barrett, A. H., \& Martin, R. N. 1978, ApJL, 221, L117

Ho, P. T. P., \& Townes, C. H. 1983, ARA\&A, 21, 239

Hüttemeister, S., Wilson, T. L., Bania, T. M., \& Martin-Pintado, J. 1993, A\&A, 280,255

Hüttemeister, S., Wilson, T. L., Mauersberger, R., et al. 1995, A\&A, 294, 667

Immer, K., Kauffmann, J., Pillai, T., Ginsburg, A., \& Menten, K. M. 2016, A\&A, 595, A94

Immer, K., Menten, K. M., Schuller, F., \& Lis, D. C. 2012, A\&A, 548, A120

Johnston, K. G., Beuther, H., Linz, H., et al. 2014, A\&A, 568, A56

Juvela, M., Padoan, P., \& Nordlund, A. 2001, ApJ, 563, 853

Kauffmann, J., Pillai, T., \& Zhang, Q. 2013, ApJL, 765, L35

Kauffmann, J., Pillai, T., Zhang, Q., et al. 2017a, A\&A, 603, A89

Kauffmann, J., Pillai, T., Zhang, Q., et al. 2017b, A\&A, 603, A90

Kendrew, S., Ginsburg, A., Johnston, K., et al. 2013, ApJL, 775, L50

Kennicutt, R. C., Jr. 1998, ApJ, 498, 541

Kruijssen, J. M. D., Dale, J. E., \& Longmore, S. N. 2015, MNRAS, 447, 1059

Kruijssen, J. M. D., \& Longmore, S. N. 2013, MNRAS, 435, 2598

Kruijssen, J. M. D., Longmore, S. N., Elmegreen, B. G., et al. 2014, MNRAS, 440, 3370

Kruijssen, J. M. D. 2017, in IAU Symp. 322, The Multi-Messenger Astrophysics of the Galactic Centre, ed. R. M. Crocker, S. N. Longmore, \& G. V. Bicknell (Cambridge: Cambridge Univ. Press), 64

Krumholz, M. R., Dekel, A., \& McKee, C. F. 2012, ApJ, 745, 69

Krumholz, M. R., \& Kruijssen, J. M. D. 2015, MNRAS, 453, 739

Krumholz, M. R., Kruijssen, J. M. D., \& Crocker, R. M. 2017, MNRAS, 466, 1213

Krumholz, M. R., \& McKee, C. F. 2005, ApJ, 630, 250

Lada, C. J., Forbrich, J., Lombardi, M., \& Alves, J. F. 2012, ApJ, 745, 190

Lada, C. J., Lombardi, M., \& Alves, J. F. 2010, ApJ, 724, 687

Larson, R. B. 1981, MNRAS, 194, 809

Launhardt, R., Zylka, R., \& Mezger, P. G. 2002, A\&A, 384, 112

Lebrón, M., Mangum, J. G., Mauersberger, R., et al. 2011, A\&A, 534, A56

Leroy, A. K., Bolatto, A. D., Ostriker, E. C., et al. 2015, ApJ, 801, 25

Leroy, A. K., Walter, F., Brinks, E., et al. 2008, AJ, 136, 2782

Lis, D. C., \& Carlstrom, J. E. 1994, ApJ, 424, 189

Lis, D. C., Li, Y., Dowell, C. D., \& Menten, K. M. 1999, in ESA Special Publication 427, The Universe as Seen by ISO, ed. P. Cox \& M. F. Kessler (Paris: ESA), 627

Lis, D. C., Menten, K. M., Serabyn, E., \& Zylka, R. 1994, ApJL, 423, L39

Longmore, S. N., Bally, J., Testi, L., et al. 2013a, MNRAS, 429, 987

Longmore, S. N., Kruijssen, J. M. D., Bally, J., et al. 2013b, MNRAS, 433, L15

Longmore, S. N., Rathborne, J., Bastian, N., et al. 2012, ApJ, 746, 117

Lu, X., Zhang, Q., Kauffmann, J., et al. 2017, in IAU Symp. 322, The MultiMessenger Astrophysics of the Galactic Centre, ed. R. M. Crocker, S. N. Longmore, \& G. V. Bicknell (Cambridge: Cambridge Univ. Press), 99
Mangum, J. G., Darling, J., Henkel, C., et al. 2013, ApJ, 779, 33

Mauersberger, R., Henkel, C., Weiß, A., Peck, A. B., \& Hagiwara, Y. 2003, A\&A, 403, 561

Mauersberger, R., Henkel, C., Wilson, T. L., \& Walmsley, C. M. 1986, A\&A, 162, 199

McGary, R. S., Coil, A. L., \& Ho, P. T. P. 2001, ApJ, 559, 326

McMullin, J. P., Waters, B., Schiebel, D., Young, W., \& Golap, K. 2007, in ASP Conf. Ser. 376, Astronomical Data Analysis Software and Systems XVI, ed. R. A. Shaw, F. Hill, \& D. J. Bell (San Francisco, CA: ASP), 127

Mills, E. A. C., \& Morris, M. R. 2013, ApJ, 772, 105

Molinari, S., Bally, J., Noriega-Crespo, A., et al. 2011, ApJL, 735, L33

Morris, M., \& Serabyn, E. 1996, ARA\&A, 34, 645

Morris, M., Zuckerman, B., Palmer, P., \& Turner, B. E. 1973, ApJ, 186, 501

Oka, T., Hasegawa, T., Sato, F., Tsuboi, M., \& Miyazaki, A. 1998, ApJS, 118,455

Oka, T., Mizuno, R., Miura, K., \& Takekawa, S. 2016, ApJL, 816, L7

Ott, J., Henkel, C., Braatz, J. A., \& Weiß, A. 2011, ApJ, 742, 95

Ott, J., Weiss, A., Henkel, C., \& Walter, F. 2005, ApJ, 629, 767

Ott, J., Weiß, A., Staveley-Smith, L., Henkel, C., \& Meier, D. S. 2014, ApJ, 785,55

Ott, J., Weiß, A., Staveley-Smith, L., Henkel, C., \& Meier, D. S. 2014, ApJ, 785,55

Pillai, T., Kauffmann, J., Tan, J. C., et al. 2015, ApJ, 799, 74

Rathborne, J. M., Longmore, S. N., Jackson, J. M., et al. 2014a, ApJ, 786, 140

Rathborne, J. M., Longmore, S. N., Jackson, J. M., et al. 2014b, ApJL, 795, L25

Rathborne, J. M., Whitaker, J. S., Jackson, J. M., et al. 2016, PASA, 33, e030

Reid, M. J., Menten, K. M., Brunthaler, A., et al. 2014, ApJ, 783, 130

Ridley, M. G. L., Sormani, M. C., Treß, R. G., Magorrian, J., \& Klessen, R. S. 2017, MNRAS, 469, 2251

Robitaille, T., \& Bressert, E. 2012, APLpy: Astronomical Plotting Library in Python, Astrophysics Source Code Library, ascl:1208.017

Rodríguez, L. F., \& Zapata, L. A. 2013, ApJL, 767, L13

Rodríguez-Fernández, N. J. 2011, MSAIS, 18, 195

Rodríguez-Fernández, N. J., \& Combes, F. 2008, A\&A, 489, 115

Sault, R. J., Staveley-Smith, L., \& Brouw, W. N. 1996, A\&AS, 120, 375

Sault, R. J., Teuben, P. J., \& Wright, M. C. H. 1995, in ASP Conf. Ser. 77, Astronomical Data Analysis Software and Systems IV, ed. R. A. Shaw, H. E. Payne, \& J. J. E. Hayes (San Francisco, CA: ASP), 433

Sawada, T., Hasegawa, T., Handa, T., \& Cohen, R. J. 2004, MNRAS, 349, 1167

Schilke, P. 1989, dissertation, Universität Bonn

Schuller, F., Menten, K. M., Contreras, Y., et al. 2009, A\&A, 504, 415

Shetty, R., Beaumont, C. N., Burton, M. G., Kelly, B. C., \& Klessen, R. S. 2012, MNRAS, 425, 720

Shirley, Y. L. 2015, PASP, 127, 299

Sofue, Y. 1995, PASJ, 47, 527

Stolte, A., Hußmann, B., Morris, M. R., et al. 2014, ApJ, 789, 115

Takekawa, S., Oka, T., \& Tanaka, K. 2015, in ASP Conf. Ser. 499, Revolution in Astronomy with ALMA: The Third Year, ed. D. Iono et al. (San Francisco, CA: ASP), 171

Townes, C. H., \& Schawlow, A. L. 1975, Microwave Spectroscopy (New York: Dover)

Ungerechts, H., Walmsley, C., \& Winnewisser, G. 1986, A\&A, 157, 207

Walker, D. L., Longmore, S. N., Bastian, N., et al. 2015, MNRAS, 449, 715

Walmsley, C., \& Ungerechts, H. 1983, A\&A, 122, 164

Wilson, W. E., Ferris, R. H., Axtens, P., et al. 2011, MNRAS, 416, 832

Yusef-Zadeh, F., Wardle, M., \& Roy, S. 2007, ApJL, 665, L123

Zschaechner, L. K., Ott, J., Walter, F., et al. 2016, ApJ, 833, 41 